\title{
6 Radiation Chemistry of Solid-State Carbohydrates Using EMR
}

\author{
H. Vrielinck, H. De Cooman, F. Callens \\ Ghent University, Dept. Solid State Sciences, Krijgslaan 281-S1, B-9000 Gent, Belgium
}

\section{E. Sagstuen}

University of Oslo, Dept. Physics, P.O.Box 1048 Blindern, N-0316 Oslo, Norway

\begin{abstract}
We review our research of the past decade towards identification of radiation-induced radicals in solid state sugars and sugar phosphates. Detailed models of the radical structures are obtained by combining EPR and ENDOR experiments with DFT calculations of $g$ and proton HF tensors, with agreement in their anisotropy serving as most important criterion. Symmetry-related and Schonland ambiguities, which may hamper such identification, are reviewed. Thermally induced transformations of initial radiation damage into more stable radicals can also be monitored in the EPR (and ENDOR) experiments and in principle provide information on stable radical formation mechanisms. Thermal annealing experiments reveal, however, that radical recombination and/or diamagnetic radiation damage is also quite important. Analysis strategies are illustrated with research on sucrose. Results on dipotassium glucose-1-phosphate and trehalose dihydrate, fructose and sorbose are also briefly discussed. Our study demonstrates that radiation damage is strongly regio-selective and that certain general principles govern the stable radical formation.
\end{abstract}

\subsection{Introduction and motivation of the study}

The structure of radiation-induced radicals in solid state sucrose has been studied with electron magnetic resonance (EMR) techniques since the early 1960's [1-7]. However, only some five years ago the identity of three radicals, dominating the room temperature (RT) stable electron paramagnetic resonance (EPR) spectrum, was convincingly established $[8,9]$. This resulted primarily from a detailed comparison of proton hyperfine (HF) interactions, determined from single crystal electron nuclear double resonance (ENDOR) experiments, with the results of highlevel density functional theory (DFT) calculations. The situation for other sugars and related carbohydrates is similar: although stable radical production has been reported for several decades, reliable identifications of their structures are in gen- 
eral much more recent [10-12] or has to date not yet been achieved [13,14]. A first important reason for this is that stable radical structures in organic solids very often differ substantially from the pristine molecule (crystal) structures and are the result of a complex reaction chain after the initial ionization or electron capture process [15]. The radicals stabilized after irradiation at low temperatures ( liquid $\mathrm{He}$ or $\mathrm{N}_{2}$ ) are, however, more directly structurally related to those of the pristine molecules, and although such studies are experimentally far more challenging, the identification of the radical species is therefore in principle simpler [16-21]. Following the evolution of the EPR and ENDOR spectra towards the RT stable stage via thermal annealing is one approach to elucidate the structures of the stable species and to unveil their formation mechanisms. Irradiation in general, however, produces several distinct radical species whose EPR spectra, all characterized by HF interactions with several protons, strongly overlap. This is the second main reason why EPR spectra of irradiated sugars are often difficult to interpret, even when studied in single crystal form. In this chapter, techniques for spectrum decomposition and strategies for identification of structure and formation pathways for (stable) radicals are illustrated using our studies on solid state sucrose and related carbohydrates.

The main goal of this research is fundamental: improving the understanding of the radiation chemistry in solid carbohydrates. Important research objectives include uncovering general principles in the radiation-induced reactions and understanding the selectivity of radical formation in these materials. First and foremost, this requires reliable identification of the radicals in a sufficiently large number of materials and at different stages of the post-radiation chemistry. The results of this study may furthermore help to elucidate the direct radiation effect in the sugar units of the DNA helix [22,23]. The latter is of considerable importance as sugar radicals are the main precursors for double strand breaks, the most harmful form of DNA damage with respect to biological consequences. Although not a priori obvious, solid state sugars may present a good model for studying direct effect radiation-induced damage in DNA as there are no radical interactions with a solvent. Moreover, the crystal structure to some extent mimics the tight, rigid packing of DNA in chromatin, and the role of hydrogen bonds in both systems is expected to be similar. As the similarity between DNA and solid state sugars may include both the initial radiation damage and the stable products, information on structures and processes at all stages after irradiation is relevant.

A second motivation is more applied and mostly related with the final stable radicals. The EPR signal intensity of such radicals may be used to determine radiation doses. Since the beginning of the 1980s the potential of sucrose in radiation dosimetry has been recognized $[24,25]$ and has been the subject of many EPR studies [26-32]. When samples are properly stored, the EPR spectrum of irradiated sucrose has long term stability and, after a short initial period of strong changes, shows only limited fading. Table sugar consists for over $90 \%$ of sucrose and the radiation-induced EPR spectra of table sugar and sucrose resemble each other very closely. Therefore, the ubiquitous table sugar is of particular interest for accident and emergency dosimetry. The composite nature of the stable EPR spectrum of ir- 
radiated sucrose, as a result of the presence of various types of radicals along with the complex transformations of this spectrum in a short time span after irradiation, are issues of concern. Sucrose is not the only sugar interesting from a dosimetric point of view. One can also apply EPR spectrometry for detection and control of irradiated sugar-containing foodstuffs [33-42]. Here, however, an additional complication arises. In foodstuffs very often a mixture of sugars is present, all giving rise to distinct radiation-induced EPR spectra. In this respect achieving a detailed understanding of the spectral shape of various common sugars, e.g. glucose, fructose and sorbose, is very relevant.

\subsection{Materials and experimental methods}

\subsubsection{Solid state carbohydrates}

Table 6.1. Overview of carbohydrates investigated in this work, labeling the $\mathrm{C}$-atoms. $\beta$ indicates the angle between the $\langle a\rangle$ and the $\langle c\rangle$ direction for monoclinic lattices.

\begin{tabular}{|c|c|c|c|}
\hline Chemical name & Short name & Molecular structure & Space group \\
\hline $\begin{array}{l}\alpha \text {-D-glucopyranosyl- } \\
(1 \leftrightarrow 2)-\beta \text {-D- } \\
\text { fructofuranoside }\end{array}$ & sucrose & & $\begin{array}{l}\mathrm{P} 2{ }_{1}, \text { monoclinic, } \\
\beta=102.97^{\circ}\end{array}$ \\
\hline $\begin{array}{l}\text { Dipotassium glucopyra- } \\
\text { nose-1-phosphate }\end{array}$ & $\mathrm{K} 2 \mathrm{G} 1 \mathrm{P}$ & & $\begin{array}{l}\mathrm{P} 2_{1}, \text { monoclinic, } \\
\beta=110.39^{\circ}\end{array}$ \\
\hline $\begin{array}{l}\alpha \text {-D-glucopyranosyl- } \\
(1 \leftrightarrow 1)-\alpha \text {-D- } \\
\text { glucopyranoside }\end{array}$ & trehalose & & $\begin{array}{l}\mathrm{P} 22_{1} 2_{1} \text {, ortho- } \\
\text { rhombic }\end{array}$ \\
\hline$\beta$-D-fructopyranose & fructose & & $\begin{array}{l}\mathrm{P} 2_{1} 2_{1} 2_{1} \text {, ortho- } \\
\text { rhombic }\end{array}$ \\
\hline$\alpha$-L-sorbopyranose & sorbose & & $\begin{array}{l}\mathrm{P} 22_{1} 2_{1} 2_{1} \text {, ortho- } \\
\text { rhombic }\end{array}$ \\
\hline
\end{tabular}


Table 6.1 gives an overview of the carbohydrates that have been covered by our studies. Most of the experiments were performed on single crystals, grown from aqueous solution by slow evaporation of the solvent at constant temperature. When possible, partially deuterated crystals were also grown from $\mathrm{D}_{2} \mathrm{O}$ solutions. Crystallization from $\mathrm{D}_{2} \mathrm{O}$, redissolution in $\mathrm{D}_{2} \mathrm{O}$ and recrystallization is expected to replace all the crystal water by $\mathrm{D}_{2} \mathrm{O}$ and $95 \%$ of the $\mathrm{OH}$ groups by OD. For fructose, crystallization proved very difficult, rendering deuteration unfeasible.

Selected experiments were also performed on irradiated powders of carbohydrates. These were used as purchased, without further refinement. In the case of fructose, selectively ${ }^{13} \mathrm{C}$ and ${ }^{2} \mathrm{H}$ labeled carbohydrate powders (Sigma Aldrich, Omicron Chemicals) were included in the study.

Crystals were oriented for rotation around a crystallographic axis, either by Laue diffraction or by inspection of the X-ray diffraction pole figures and then transferred to sample holders (quartz rods or copper pedestals) or fixed in quartz tubes with minimal loss of orientation $\left(<5^{\circ}\right)$. For the crystals with orthorhombic symmetry these rotation axes are usually $\langle a\rangle,\langle b\rangle$ and $\langle c\rangle$. For sugars (sugar phosphates) with monoclinic symmetry crystals were at some occasions oriented for rotation around the $\langle a *\rangle$ (perpendicular to $\langle b\rangle$ and $\langle c\rangle$ ) or $\langle c *\rangle$ axes. In order to resolve the Schonland ambiguity [43, 44], the angular dependence of the EPR and ENDOR spectra was also recorded for rotations around axes deviating from those mentioned above. These rotation planes will be referred to as skewed planes.

\subsubsection{Irradiation for radical production}

Irradiations either at RT or at $273 \mathrm{~K}$ (water-ice cooled sample) occurred ex situ, using X-rays from a Philips $\mathrm{W}$-anode X-ray tube operated at $60 \mathrm{kV}$ and $40 \mathrm{~mA}$, resulting in a radiation dose rate of approximately $1.3 \mathrm{kGy} / \mathrm{min}$. For most ENDOR experiments the samples needed to be irradiated for at least $1 \mathrm{~h}$. In the study of stable radicals, crystals were irradiated before orienting and mounting onto quartz sample holders. In the study of less stable species samples had to be irradiated after mounting in quartz holders and EPR spectra sometimes contained contributions from irradiated quartz. After irradiation, crystals or powders were transferred into a temperature-controlled (by Oxford He flow cryostats) microwave cavity, stabilized at the measurement temperature.

For studying primary radical species and/or intermediate steps in the evolution towards RT stable radiation products, crystals were irradiated in situ at 6-8 K using liquid helium or at $77 \mathrm{~K}$ using liquid nitrogen. For this purpose, a special microwave cavity assembly originally devised by W.H. Nelson was constructed [45]. The setup for X-band $(9.8 \mathrm{GHz})$ is shown in Fig. 6.1. 

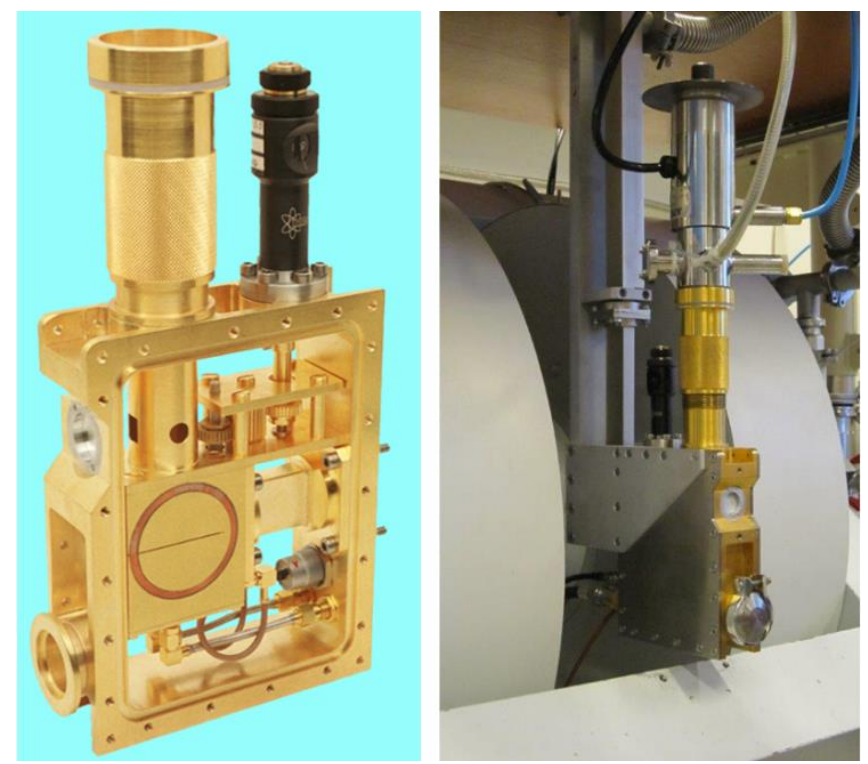

Fig. 6.1. X-band EPR/ENDOR cavity for in situ X-irradiation of crystals at low temperature. Left : detail of the cavity design with the vacuum-sealed side-walls of the system removed for showing the EPR modulation coils, the iris adjustment system, optical and X-ray irradiation windows, electrical connections for the EPR modulation and the (internal) 4-turn ENDOR coils, the waveguide connection and a vacuum pump-out port, right : cavity in the setup - microwave bridge and cavity can be slid between and outside of the poles of the electromagnet, for measurement and X- or UV irradiation, respectively.

A copper sample rod to which the crystal is mounted with conducting epoxy glue is fixed to the Joule-Thompson end of a cold-finger cryostat (Air Products HeliTran). This is inserted into a telescoping cryostat holder at the top of an evacuated titanium/brass enclosure. The enclosure is equipped with a thin $\mathrm{Al}$ and a quartz window to allow for X-ray and optical irradiation, respectively. These windows are situated above the microwave cavity. The cavities used are home-made $\mathrm{X}\left(\mathrm{TM}_{110}\right)$ or Q-band $\left(34 \mathrm{GHz}, \mathrm{TE}_{011}\right)$ cavities equipped with EPR modulationand ENDOR coils as well as externally accessible variable iris control. The cryostat with sample is lowered to the irradiation position, and after evacuation of the assembly, the crystal is cooled to the desired temperature and irradiated. The temperature is monitored and controlled using an Oxford ITC 503 temperature controller connected to a heater assembly and thermocouple at the cryostat cold end. As X-ray source, a Philips Cr-anode X-ray tube operated at $60 \mathrm{kV}$ and $40 \mathrm{~mA}$ was used, providing a radiation dose rate of approximately $0.16 \mathrm{kGy} / \mathrm{min}$. For the results presented here, doses between 10 and $40 \mathrm{kGy}$ were used. After irradiation, the sample is lowered into the center of the microwave cavity for EMR measurements. Oxygen-free high conductivity copper sample posts and conducting epoxy glue are used to avoid EPR signals from radiation-induced radicals in the crystal mounting system. 


\subsubsection{EPR, ENDOR and ENDOR-induced EPR experiments}

The EMR experiments were performed using commercial Bruker CW spectrometers (ER200, Elexsys 500/560) at the microwave X- and Q-bands. For $g$ factor determinations, accurate measurements of the microwave frequency and magnetic field are indispensable. The frequency measurements were achieved using external $\mathrm{X}$ and Q-band frequency counters.

For the EMR measurements after RT irradiation, the magnetic field at the beginning and at the end of each field sweep was measured using an ER035M NMR Teslameter. Subsequently a calibration of the field measurements was performed by determining the $g$ value of a field marker (DPPH at 2.0036 [46] in X-band, $g_{\perp}$ of $\mathrm{CO}_{3}{ }^{3-}$ in $\mathrm{CaCO}_{3}$ at 2.0031 [47] in Q-band). For the EMR measurements after in situ low temperature (LT) irradiation, the magnetic field was calibrated using a BRUKER ER036 TM Teslameter in combination with a DPPH field marker.

All EPR spectra presented here are recorded using magnetic field modulation (100 kHz, $0.1 \mathrm{mT}$, unless otherwise mentioned) and all ENDOR spectra using frequency modulation (modulated at $10,12.5$ or $25 \mathrm{kHz}$ with a few $100 \mathrm{kHz}$ depth). As a result their shape resembles the first derivative of an absorption spectrum. Occasionally, pulsed EPR experiments at X-band (Hyperfine Sublevel Correlation Spectroscopy = HYSCORE [48]) were performed in collaboration with other research groups: we refer to the relevant paper for experimental details [9].

ENDOR normally requires partial saturation of the EPR spectrum. Although EPR spectra of radiation-induced radicals in carbohydrates readily saturate at any temperature for microwave powers above $1 \mathrm{~mW}$, for ENDOR experiments very often temperatures are required that only are attainable using liquid helium $(50 \mathrm{~K}$ or $20 \mathrm{~K}$ ). For sucrose at X-band, however, ENDOR spectra can be recorded at any temperature from 4 to $300 \mathrm{~K}$.

ENDOR measurements for carbohydrates in general only reveal HF interactions with protons, the larger of which are resolved in the EPR spectra. Yet, a detailed ENDOR analysis is necessary as the EPR spectra are very complex. The simultaneous observation of spectra from several symmetry-related orientations with respect to the magnetic field - of the same radical species [49], and this for several different radicals present, makes an EPR-only analysis practically impossible. ENDOR is usually performed by saturating the EPR spectrum at a fixed magnetic field position, sweeping the radiofrequency. In order to obtain the transitions for all dominant radical species and all sites, for each magnetic field orientation, the ENDOR spectrum has to be recorded at several (very often 2-3) field positions for each orientation. Nonetheless, certain (probably less dominant) radical species may escape from detection in this way. In order to avoid this, one can record the ENDOR spectrum as a function of magnetic field over the complete range where EPR intensity is observed $[50,51]$. 
As an example of such an ENDOR experiment carried out in the fieldfrequency space (also called Field-Frequency ENDOR, FF-ENDOR), a FFENDOR spectrum of sucrose, recorded at $110 \mathrm{~K}$ several days after X-ray irradiation at RT, is shown in Fig. 6.2. This spectrum is obtained with the magnetic field oriented close to the $\langle b\rangle$ axis. Along with the FF-spectrum, corresponding ENDOR, EPR and ENDOR-induced EPR (EIE) traces are shown. The signal height is represented in color scale in the FF-ENDOR spectrum, with red and blue indicating high and low (negative) values, respectively. The intensity of the spectra is increased in order to enhance the contrast for the signals of the more informative larger proton HF couplings, and as a result in the proton Larmor frequency range (50-52 $\mathrm{MHz}$ at Q-band) only one broad, intense line can be discerned. The horizontal dashed line in Fig. 6.2 corresponds to an ENDOR spectrum recorded at one magnetic field position $(1212.5 \mathrm{mT})$ which is shown at the top of the figure. Combinations of ENDOR transitions at particular radiofrequencies $\left(v_{E N D O R}\right)$ are repeated at several magnetic field positions, although the transition frequencies exhibit a slight shift with magnetic field $(B)$. This shift may be predicted to first order as (see also Section6.2.4, spin Hamiltonian = SH) [49]

$$
\frac{\partial v_{E N D O R}}{\partial B}= \pm g_{H} \mu_{N}
$$

with $g_{H}$ the proton nuclear $g$ factor and $\mu_{N}$ the nuclear magneton. For nuclei with $I=1 / 2$ all ENDOR transitions can be recorded at each EPR transition of a particular radical, hence one can in principle reconstruct the EPR spectrum corresponding to a particular ENDOR transition by measuring its signal height at a maximum of the (first derivative) signal as a function of magnetic field while simultaneously correcting the transition frequency according to Equation (6.1). Such spectra are referred to as EIE spectra [52-54]. In the bottom of the figure, examples of the EIE spectra recorded are shown, by monitoring the height of the ENDOR lines marked in the top spectrum of Fig. 6.2 while sweeping the magnetic field. As we follow the positive peak position, the EIE spectrum is expected to have an absorption-like shape, as opposed to the first-derivative shape for the EPR spectrum. Although EPR and EIE spectra of a radical should carry the same SH information, an EIE spectrum cannot be expected to perfectly match the integrated EPR spectrum of the corresponding radical component. Indeed, this would imply that i) there are no higher order field corrections to the ENDOR frequencies, ii) the width (shape) of the ENDOR line remains constant throughout the complete EPR field range and iii) the EPR and ENDOR spectra are by no means distorted by the electron and/or nuclear spin relaxation. In particular, it should be remembered that the EIE is recorded under EPR microwave saturation conditions commonly resulting in significant line broadening. Nevertheless, EIE is very effective in decomposing multicomposite EPR spectra and is less time-consuming than recording the FF-ENDOR spectrum over the complete EPR field range. 

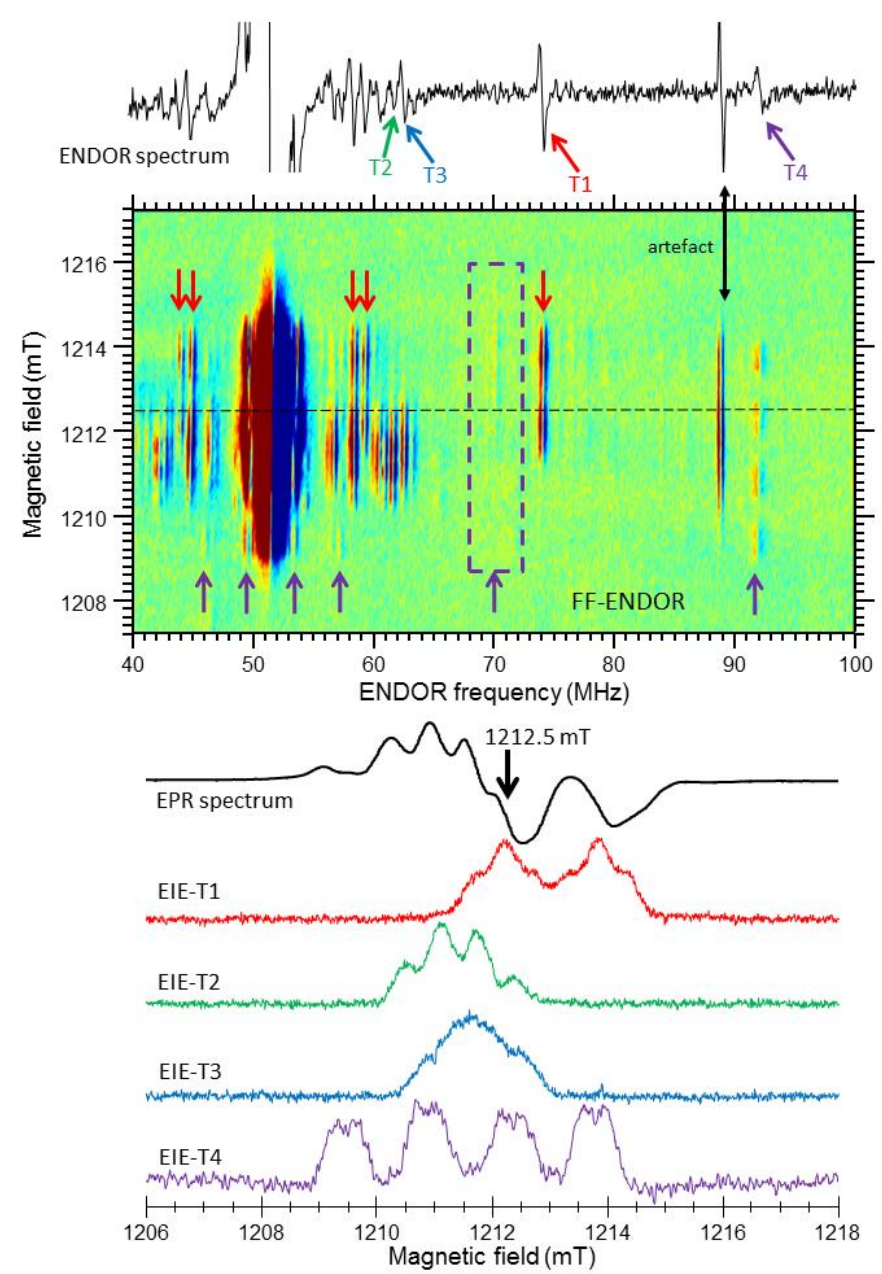

Fig. 6.2. ENDOR, FF-ENDOR, EPR and EIE spectra of stable radical species in sucrose Xirradiated at RT and, recorded at $110 \mathrm{~K}$ with the magnetic field parallel to the $\langle b\rangle$ axis, several days after irradiation. The ENDOR spectrum is recorded at the magnetic field position marked with an arrow in the EPR spectrum and corresponds to the horizontal cut in the FF-ENDOR spectrum indicated by the dashed line. The ENDOR transitions whose intensity is monitored in the EIE experiments (bottom traces) are marked by colored arrows. $v_{\mathrm{MW}}=34.00 \mathrm{GHz}$.

As for the interpretation of Fig. 6.2, in the FF-ENDOR spectrum four dominant contributions can be discerned. A first clear contribution, labeled T1 (red arrows), has it largest ENDOR frequency around $73 \mathrm{MHz}$, due to a $\beta$-proton HF interaction. The corresponding EIE spectrum exhibits in addition two smaller ${ }^{1} \mathrm{H}$ interactions, clearly visible with high-frequency transitions slightly below $60 \mathrm{MHz}$ and low-frequency transitions around $45 \mathrm{MHz}$. In the $60-65 \mathrm{MHz}$ range, the two over- 
lapping transitions of the strongest, $\alpha$-type HF interactions of radicals T2 and T3 are seen. Also these two radicals have two more pronounced HF interactions, which are strongly overlapping at this particular field orientation and for this reason are not indicated in the FF-ENDOR spectrum. The T1-T3 radicals have been thoroughly characterized through angular dependent EPR and ENDOR measurements [55] and detailed molecular models were found for them, based on DFT calculations (see Section 6.3.4) $[8,9]$. They constitute the dominant contribution to the central part of the powder EPR spectrum of irradiated sucrose (see Section 6.4.5).

Above $90 \mathrm{MHz}$, another interesting contribution to the spectrum is discerned. It is labeled here as T4 [56] and its ENDOR transitions are marked by purple arrows in the FF-ENDOR spectrum. The corresponding EPR (EIE) spectrum exhibits three resolved HF interactions. The largest splitting $(2.9 \mathrm{mT}, \sim 81 \mathrm{MHz})$ corresponds to the ENDOR transition at $92-93 \mathrm{MHz}$. The ENDOR transition of the intermediate splitting $(1.4 \mathrm{mT}, \sim 37 \mathrm{MHz})$ is not clearly observed (area bordered by purple dashed line), probably as a result of line broadening. For the smallest HF interaction, only barely resolved in the EIE spectrum $(0.3 \mathrm{mT}, \sim 10 \mathrm{MHz})$, the ENDOR transitions are found at 46 and $57 \mathrm{MHz}$. The HF splitting corresponding to the transitions at 49 and $53 \mathrm{MHz}(\sim 4 \mathrm{MHz}, 1.3 \mathrm{mT})$ is not resolved in the EIE spectrum. The as yet unidentified T4 radical very probably constitutes the dominant contribution to the "wing parts" of the EPR spectrum of irradiated sucrose powder [57, 58] (see Section 6.4).

\subsubsection{Spin Hamiltonian analysis: extraction of $\mathrm{g}$ and HF tensors}

The SH relevant to the analysis of radicals in carbohydrates is that for a paramagnetic center with spin $S=1 / 2$, interacting with various nuclei - mostly protons with $\operatorname{spin} I_{i}=1 / 2[49]$

$$
\hat{H}_{S}=\mu_{B} \vec{B} \cdot \vec{g} \cdot \hat{\vec{S}}+\sum_{i}\left(\hat{\vec{S}} \cdot \vec{A}_{i} \cdot \hat{\vec{I}}_{i}-\mu_{N} g_{H} \vec{B} \cdot \hat{\vec{I}}_{i}\right)
$$

in which $\mu_{B}$ represents the Bohr magneton. $\vec{g}$ and the proton HF tensors $\vec{A}_{i}$ are to be determined by fitting the experimental angular dependence of the EPR and ENDOR spectra. In this chapter we follow the convention of labeling the principal values of these tensors $\left(g_{x}, g_{y}, g_{z} ; A_{x}, A_{y}, A_{z}\right)$ in ascending order and the corresponding principal directions are specified via their direction cosines with respect to the $\left\langle a^{(*)}\right\rangle,\langle b\rangle$ and $\langle c\rangle$ crystal axes. For radicals, which exhibit three appreciable HF interactions, like the stable radicals in sucrose (see Section 6.4.5), the SH already involves 16 states, rendering the time for computing transition fre- 
quencies (or fields) very long, especially in the context of fitting. For this reason, simplifications are used that are justified a posteriori by comparison between the experimental data and simulations based on the diagonalization of the full $\mathrm{SH}$. These approximations yield sufficiently high accuracy as long as high-field conditions are satisfied, that is, as long as the first term in the SH is at least two orders of magnitude larger than all other terms. Under these circumstances it is justified to determine the $g$ tensor for a single radical species from the center of the EPR pattern recorded at each magnetic field orientation, and thus effectively only consider the first term of the SH. The same can be done for multi-composite spectra after separating the different contributions by EIE measurements. For ENDOR experiments the high-field condition implies that coupling between the different nuclei via the electron spin is practically absent. As a result, one can analyze the HF coupling for an individual nucleus as if this is the only nuclear interaction with the unpaired electron. The sum over nuclear interactions in the SH reduces to just two terms (HF and nuclear Zeeman interaction for this one nucleus). However, it also implies that if ENDOR transitions of different symmetry-related crystal sites of the same radical are recorded in the same spectrum, e.g. as a result of insufficient $g$ resolution, it may not be evident to link HF tensors for interactions from the same site of the radical correctly. This has implications for simulations of the EPR spectra, especially for powders $[57,58]$.

The fitting of angular dependent ENDOR data was mostly performed using the MAGRES program [59]; simulations of EPR, ENDOR and EIE spectra and fitting of angular dependent EIE data were based on the EasySpin routines [60] in MATLAB. In some cases, the KVASAT program was used [61, 62]. For extracting a HF or a $g$ tensor from angular dependent ENDOR or EIE spectra, respectively, data in at least three independent planes have to be available. As mentioned in Section 6.2.1, for orthorhombic crystals very often the $\{a b\},\{b c\}$ and $\{c a\}$ planes are selected, and for monoclinic either $\left\{a^{*} b\right\},\{b c\}$ and $\left\{c a^{*}\right\}$, or $\{a b\},\left\{b c^{*}\right\}$ and $\left\{c^{*} a\right\}$. Indeed, using crystallographic rotation axes is practical, as they most often are easily recognized from the crystal morphology. In addition, a considerable advantage of taking these planes is that the magnetic field may be aligned quite easily to specific (crystallographic) orientations where different symmetry-related sites of the radicals become magnetically equivalent. At these orientations their EPR/ENDOR spectra coincide: the site-related branches in the angular variations cross. At such coincidences EPR and ENDOR spectra can be recorded with higher signal/noise ratio and they usually exhibit an easier substructure. However, an analysis in these three planes in general yields two $g$ or HF tensors that fit the available data equally well. This phenomenon, that has been labeled as the "Schonland ambiguity" [43, 44], is well-established in literature but still not widely recognized among experimental and computational researchers. Only one of the two fitting results corresponds to the actual tensor to be determined, and also fits the data that may be obtained outside of these three planes. The second tensor (labeled "Schonland conjugate") is merely a fitting result without further physical 
meaning. For the principles of this ambiguity in $g$ and HF tensor analysis, as well as for practical ways of avoiding it, we refer to our recent paper on this subject [44]. In the next subsection, the Schonland ambiguity is illustrated in the analysis procedure for a particular example.

\subsubsection{Example of Schonland ambiguity in data analysis}

The Schonland ambiguity finds its origin in an ambiguity in the choice of rotation sense when analyzing angular dependent data. We illustrate the principles here using the $\mathrm{SH}$ data for the stable radical $\mathrm{T} 1$ in irradiated sucrose. This is not because it would be the best example, but rather in order to demonstrate that the Schonland ambiguity may pop up in any $g$ or HF tensor analysis when data are restricted to three orthogonal planes defined by the unit cell axes. As already mentioned, sucrose has monoclinic symmetry. The analysis is presented for data in the $\left\{a^{*} b\right\}$, $\{b c\}$ and $\left\{c a^{*}\right\}$ planes, and the SH parameters (tensors) are represented in the $a^{*} b c$ reference frame. Implications of having data in other (non-orthogonal) rotation planes for calculating Schonland conjugate tensors and/or lifting Schonland ambiguity may be found in our basic article on this subject [44].

For most of the radicals discussed here, the HF tensors were analyzed before the $g$ tensors, which were extracted from EIE measurements and relied on the knowledge of the HF tensors $[13,57,63]$. It is more instructive, however, to start the discussion with the problem of extracting a $g$ tensor from angular dependent resonance field positions. In Fig. 6.3 simulated angular dependences of the central field position of the EPR (or EIE) spectrum of T1 are shown in four rotation planes [57]. These make use of the $g$ tensor data in Table $6.2:$ the $g$ tensors for the two (monoclinic) symmetry-related radicals (Tensors 1 and 2) and the two tensors Schonland conjugated to these (Tensors 3 and 4). In Ref. [44] it is shown that the latter can be calculated by changing either the $(1,2)$ and $(2,1)$, or the $(2,3)$ and $(3,2)$ elements of the symmetric tensor $\vec{g}^{2}=\vec{g} \cdot \vec{g}^{T}$. Symmetry-related tensors only differ with respect to their principal directions, whereas Schonland conjugate tensors also differ with respect to the principal values. In Table 6.2 one may verify that in the chosen example this difference in principal $g$ values is subtle (yet not negligible), but that the differences in principal directions may be quite substantial.

Carbon-centered radicals in carbohydrates often exhibit close to axial $g$ tensor symmetry $\left(g_{x}<g_{y} \approx g_{z}\right)$. The $g$ tensor of the T1 radical is, however, clearly rhombic, with three clearly different principal values, two of which exhibit a considerable positive shift from the free electron $g$ value. The implications of these characteristics for the radical model will be discussed in Section 6.3.4. 
Table 6.2. $g$ tensor for the $\mathrm{T} 1$ stable radical in irradiated sucrose [57]: tensor 1 - original tensor as obtained from fitting angular dependent EIE data ; tensor 2 - monoclinic symmetry-related tensor to 1 ; tensor 3 - Schonland conjugate of tensor 1 by inverting the $g^{2}(1,2)$ and $g^{2}(2,1)$ matrix elements of tensor 1 ; tensor 4 - monoclinic symmetry-related tensor to 3 . In the last two columns, the difference in principal values and directions (smallest angle between the directions, in degrees) with tensor 1 are given.

\begin{tabular}{ccccccc}
\hline Tensor \# & $g_{i}$ & $\langle a *\rangle$ & $\langle b>$ & $\langle c\rangle$ & $\Delta g_{i}$ & $\Delta \theta$ \\
\hline \multirow{2}{*}{1} & 2.0021 & 0.3619 & -0.9232 & -0.1295 & & \\
& 2.0049 & 0.8781 & 0.2991 & 0.3798 & & \\
& 2.0066 & -0.3130 & -0.2512 & 0.9160 & & \\
\multirow{2}{*}{2} & 2.0021 & -0.3619 & -0.9232 & 0.1295 & 0 & 45 \\
& 2.0049 & 0.8781 & -0.2991 & 0.3798 & 0 & 34 \\
& 2.0066 & -0.3130 & 0.2512 & 0.9160 & 0 & 29 \\
\multirow{2}{*}{3} & 2.0020 & -0.3862 & -0.9030 & -0.1884 & -0.0001 & 44 \\
& 2.0051 & 0.9156 & -0.4001 & 0.0405 & 0.0002 & 45 \\
& 2.0065 & -0.1120 & -0.1569 & 0.9813 & -0.0001 & 13 \\
& 2.0020 & 0.3862 & -0.9030 & 0.1884 & -0.0001 & 18 \\
\multirow{2}{*}{4} & 2.0051 & 0.9156 & 0.4001 & 0.0405 & 0.0002 & 21 \\
& 2.0065 & -0.1120 & 0.1569 & 0.9813 & -0.0001 & 27 \\
\hline
\end{tabular}

In Fig. 6.3 X-band simulations using Tensors 1 and 2 are represented with symbols and could be considered as experimental data. The simulations with the other two tensors are shown as full lines.

The example nicely illustrates that when restricting measurements to the first three planes, one can find two tensors, not related by symmetry, which fit the data equally well. The Schonland ambiguity springs from the fact that one cannot $a$ priori know which symmetry-related branches in the different rotation planes to connect with one another. It is, however, also immediately clear that in the fourth skewed plane the degeneracy of the Schonland conjugate tensors is lifted, presenting an obvious method to select the right set of tensors among the two possibilities.

The simulations at the bottom of Fig. 6.3 suggest that inspection of the powder spectrum may also be used for resolving the Schonland ambiguity. This may in particular be so if line widths are narrow and if the spectra are not complicated by resolved HF interactions. Powder spectra are particularly sensitive to changes in the rhombicity of $g$ tensors. This results in a considerable difference in the low field part of the simulated powder EPR spectra for the two Schonland conjugate tensors, in spite of only very subtle changes in the absolute $g$ values. 

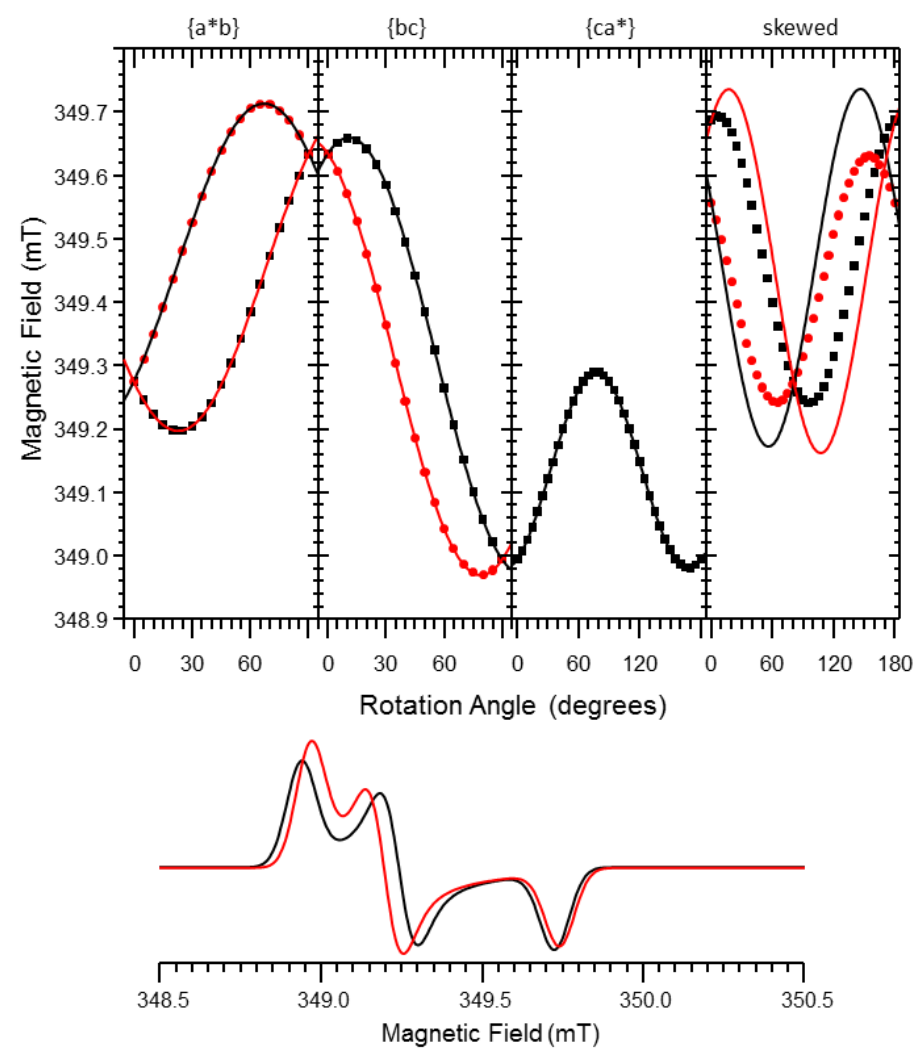

Fig. 6.3. Illustration of Schonland ambiguity for the $g$ tensor for data analysis in three orthogonal $\left(\left\{a^{*} b\right\},\{b c\}\right.$ and $\left.\left\{c a^{*}\right\}\right)$ and one skewed plane (polar angles $\left.\theta=26.1^{\circ}, \phi=190.3^{\circ}\right)$. The $g$ tensors used in the simulations are listed in Table 6.2 : filled black squares - tensor 1 ; filled red circles - tensor 2 ; full black line - tensor 3 ; full red line - tensor 4 . In the bottom part of the figure the simulated powder EPR spectra are shown, assuming that no HF interactions are resolved, using the original $g$ tensor (tensor 1 or tensor 2 - black line) and its Schonland conjugate (tensor 3 or tensor 4). The polar angles are defined such that $\langle c\rangle$ corresponds with $\theta=0^{\circ}$ and $\left\langle a^{*}\right\rangle$ with $\theta=90^{\circ}, \phi=0^{\circ} . v_{\mathrm{MW}}=9.8 \mathrm{GHz}$.

We now move on to the analysis of HF tensors from angular dependent ENDOR measurements. The three HF tensors determined for the T1 radical in irradiated sucrose [55], along with their Schonland conjugates, are taken as an example. Their principal values and directions are given in Table 6.3, for only one of the symmetry-related sites. The calculation of a Schonland conjugate $A$ tensor requires more care than in the case of the $g$ tensor, however. The result does not only depend on the rotation planes in which experimental data were analyzed (as for the $g$ tensor), but also on the microwave frequency (magnetic field $B_{0}$ at which the ENDOR spectrum is recorded) and in which of the $M_{S}$ electron spin multiplets the 
ENDOR transitions take place. Very often only the high-frequency branches (with $M_{S}=-1 / 2(1 / 2)$ for $\left.A>0(A<0)\right)$ of ENDOR transitions are considered for extracting the HF tensor. The low-frequency transitions are in many cases not (well) observed and for that reason sometimes simply not recorded. In Ref. [44] it is demonstrated that in this particular case one can find the tensor Schonland conjugate to a given tensor $\vec{A}$ by making a similar sign change in the off-diagonal elements (as for the $g$ tensor) of the symmetric tensor $\overleftrightarrow{K}_{\text {high }}^{2}$

$$
\vec{K}_{\text {high }}=-\operatorname{sign}\left(A_{\text {iso }}\right) \frac{\vec{A}}{2}-g_{N} \mu_{N} B_{0} \overrightarrow{1}
$$

with $A_{i s o}=\operatorname{trace}(\overleftrightarrow{A}) / 3$, the isotropic HF coupling.

Table 6.3. Principal $A$ values and directions for the three resolved proton HF interactions of the T1 stable radical in irradiated sucrose, as determined from angular dependent ENDOR measurements in four rotation planes (HF1, HF2, HF3) [9] and the Schonland conjugate tensors (HF1S, HF2S and HF3S) which fit the data for the high-frequency X-band ENDOR branches in the $\left\{a^{*} b\right\},\{b c\}$ and $\left\{c a^{*}\right\}$ planes equally well. The last two columns present the differences in principal values and directions (angles in degrees) between Schonland conjugate and original tensors.

\begin{tabular}{|c|c|c|c|c|c|c|}
\hline$A$ tensor & $A_{i}$ & $<a *>$ & $<b>$ & $<c>$ & $\Delta A_{i}$ & $\Delta \theta$ \\
\hline \multirow{3}{*}{ HF1 } & 42.81 & 0.616 & 0.121 & -0.778 & & \\
\hline & 44.42 & 0.072 & 0.975 & 0.209 & & \\
\hline & 53.17 & 0.784 & -0.185 & 0.592 & & \\
\hline \multirow{3}{*}{ HF1S } & 41.90 & 0.547 & 0.499 & -0.673 & -0.91 & 23 \\
\hline & 45.56 & -0.246 & 0.863 & 0.441 & 1.14 & 24 \\
\hline & 52.92 & 0.800 & -0.075 & 0.595 & -0.24 & 7 \\
\hline \multirow{3}{*}{ HF2 } & 13.19 & 0.106 & 0.825 & 0.555 & & \\
\hline & 13.57 & 0.989 & -0.144 & 0.025 & & \\
\hline & 20.87 & 0.101 & 0.546 & -0.832 & & \\
\hline \multirow{3}{*}{ HF2S } & 12.66 & 0.595 & 0.658 & 0.461 & -0.53 & 30 \\
\hline & 14.15 & 0.803 & -0.519 & -0.294 & 0.58 & 31 \\
\hline & 20.81 & 0.046 & 0.545 & -0.837 & -0.06 & 3 \\
\hline \multirow{3}{*}{ HF3 } & -17.48 & -0.711 & -0.241 & 0.660 & & \\
\hline & -14.80 & -0.38 & 0.922 & -0.073 & & \\
\hline & -0.93 & -0.591 & -0.302 & -0.748 & & \\
\hline \multirow{3}{*}{ HF3S } & -19.12 & -0.633 & -0.516 & 0.577 & -1.64 & 17 \\
\hline & -12.07 & -0.492 & 0.844 & 0.215 & 2.73 & 18 \\
\hline & -2.18 & -0.598 & -0.148 & -0.788 & -1.25 & 9 \\
\hline
\end{tabular}




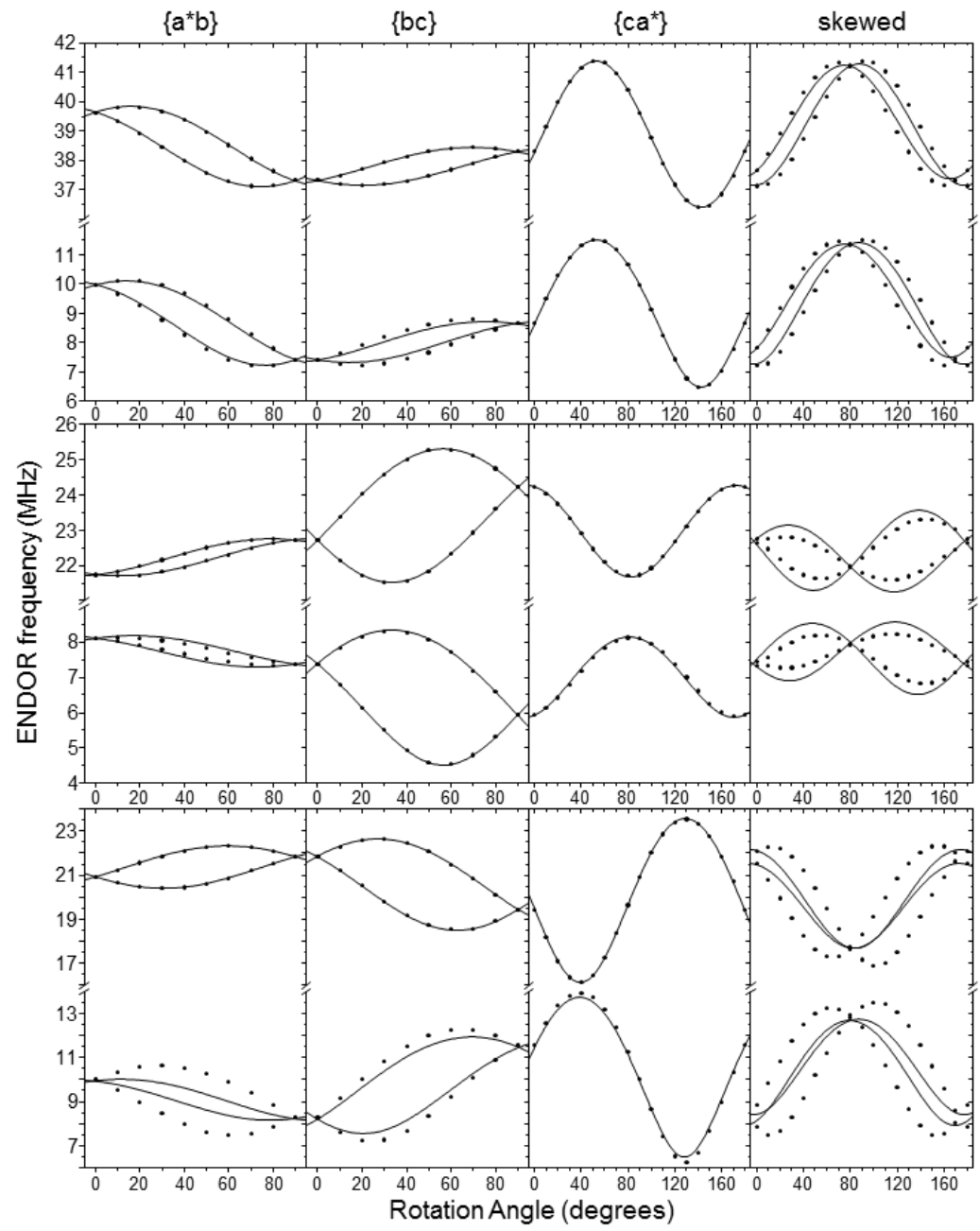

Fig. 6.4. Illustration of Schonland ambiguity for HF tensors for data analysis in three orthogonal $\left(\left\{a^{*} b\right\},\{b c\}\right.$ and $\left.\left\{c a^{*}\right\}\right)$ and one skewed plane (polar angles $\left.\theta=26.1^{\circ}, \phi=190.3^{\circ}\right)$. The HF tensors selected for simulation are those of the three resolved interactions for the stable radical T1 in irradiated sucrose (original - filled circles, and Schonland conjugate tensors - full lines), listed in Table 6.3.

Figure 6.4 is organized in a similar way as Fig. 6.3: symbols represent simulations ( experimental data points) for the original tensor and full lines for its Schonland conjugate. The simulations are again performed at X-band. Exactly as in the discussion for the $g$ tensor, for the high frequency branches the simulation for the two Schonland conjugate tensors coincide perfectly in the first three (orthogonal) planes, but in the fourth (skewed) plane a clear mismatch is observed [64]. However, Fig. 6.4 and Eq. (6.3) illustrate that the Schonland ambiguity for 
HF tensors is not as essential as in the case of the $g$ tensor. The $B_{0}$ dependence suggests that a comparison of spectra recorded in different microwave frequency bands may also resolve the ambiguity, and if one manages to record transitions in both $M_{S}$ multiplets, the ambiguity in principle does not exist [65].

The simulations for the HF1 and HF2 tensors (and their Schonland conjugates) demonstrate that in practice, the differences may be quite subtle and even lie within experimental accuracy. The differences are clearest for interaction HF3, which exhibits the largest anisotropy. In addition, in Ref. [44] we have shown that in the limiting cases for very low and very high microwave frequencies (when one of the terms in Eq. (6.3) clearly dominates), the ambiguity may also not be resolved by analyzing the data for both $M_{S}$ multiplets.

Finally, the comparison of the Schonland conjugate forms of the tensors in $\mathrm{Ta}$ bles 6.2 and 6.3 deserves some further comments. The Schonland ambiguity may hamper radical identification if the two possible fitting results may lead to different interpretations of the type of interaction. In the example elaborated in Ref. [44] it is shown how the Schonland conjugate of a particular HF tensor, typical of an $\alpha$-proton, may have the symmetry typical of a $\beta$-proton coupling with large anisotropy, as expected for e.g. a $\beta$-hydroxyl proton coupling (see Section 6.3.1).

For none of the T1 HF tensors such drastic changes in interpretation occurs, although finding the correct form for the HF3 tensor (assisted by X-band HYSCORE [9]) proved to be the key for finding the correct radical model. One may consider the differences in principal directions in Tables 6.2 and 6.3 not to be very large. However, in Section 6.3 it will be shown that the model identification strongly relies on the comparison between experimental and DFT calculated HF (and $g$ ) tensors. For accurate radical models, in particular the principal directions of calculated tensors are expected to reproduce the experimental results very well. Hence, it is important to determine these principal directions as precisely as possible and differences of the order of $\sim 10-20^{\circ}$ may indeed be very relevant.

As a conclusion of this subsection, we want to emphasize that both experimental and computational scientists involved in the identification of paramagnetic centers in low-symmetry (orthorhombic, monoclinic, triclinic) crystals should be aware of the Schonland ambiguity and its implications. Very often, having experimental data in three orthogonal planes is not sufficient to obtain an unambiguous result for the SH tensors. The discussion in this section and in Ref. [44] should enable computational scientists, aiming to find models for radicals characterized using EPR and ENDOR by others, to recognize situations where Schonland ambiguity may be an issue. Ref. [44] also provides details how to calculate the Schonland conjugate of $g$ and $A$ tensors, for cases where the wrong tensor may have been reported in the literature. Finally, it is interesting to note that similar ambiguities may arise in the analysis of EPR/ENDOR data for high-symmetry crystals. A well-known example is the ambiguity in fitting the angular dependence of the EPR spectrum for a center with trigonal symmetry in a cubic crystal in a 
$\{100\}$ plane: two $g$ tensors are found that fit the data equally well [66]. The ambiguity is lifted, however, if the angular dependence in a $\{110\}$ plane is analyzed.

\subsection{Radical model identification: from semi-empirical theory to DFT calculations}

Even when created by low temperature irradiations, bond distances and angles in the relaxed radical structures may differ significantly from those in the undamaged molecule in the crystal. This may render radical identifications based on comparisons between the experimentally determined SH parameters and the undamaged molecular structure highly uncertain. For this reason, comparison of experimental $\mathrm{SH}$ parameters with those calculated using relaxed radical geometries in many cases represent the most reliable method for identifying radical species in irradiated carbohydrates. With increasing processor power and developments in DFT methodology, such calculations have undergone a tremendous evolution in the past decades. As this chapter focuses on the experimental aspects of the study, the technical details of the calculations will not be discussed here. We will only highlight the key points in the computational evolution. For more details, we refer to our papers and to Chapter 18 of this book, devoted to this issue.

In spite of this progress, the calculations have not evolved that far that they can predict which radicals will be formed and are stable (although progress in this direction is currently being pursued). In addition, our studies have shown that stable radical structures may differ very strongly from the undamaged structure, involving not only $\mathrm{H}$-abstraction, but possibly also $\mathrm{H}_{2} \mathrm{O}$ elimination, opening of the sugar ring, glycosidic bond rupture, etc. Considering that all these events may occur at several positions in the molecule, the number of radical structures to consider in the calculations becomes very large. Fortunately, the SH parameters carry key information on the radical structure, that can be used to obtain an initial guess of the radical structure and to restrict the number of physically realistic candidates via a set of semi-empirical theoretical rules [15]. Most of the theory that relates $g$ and HF tensors of organic radicals to their electronic and geometrical structure was developed in the period 1950-1970, as experimental data became available. Very often, theoretical relations were developed for radicals with an essentially planar C-structure ( $s p^{2}$ hybridization), where the radical's unpaired electron (spin density) is mainly localized in the $2 p_{z}$ orbital of one of the C-atoms, directed perpendicular to the plane of $\mathrm{C}$ atoms. There is also very useful information available for non-planar radicals, though [67-73]. 


\subsubsection{Semi-empirical theory: information obtained from $\mathrm{HF}$ interactions}

Most of the radicals discussed in this chapter are carbon-centered with a planar bonding structure. Figure 6.5a shows a hypothetical hydroxyalkyl radical fragment that may be produced after irradiation of a carbohydrate, in which the nomenclature for proton $\mathrm{HF}$ couplings is defined. The proton bound directly to the carbon atom carrying the main spin density $\left(\mathrm{C}_{\alpha}\right)$ is called an $\alpha$-proton, a proton bound to a carbon atom adjacent to that $\left(\mathrm{C}_{\beta}\right)$ is called $\beta$-proton, on a next-nearest carbon atom $\gamma$, etc. The HF tensors of $\alpha$-protons are characterized by a negative isotropic value, $A_{i s o}^{\alpha}$, due to spin polarization, and a strong anisotropy with a characteristic rhombic pattern [74]

$$
\vec{A}_{\text {aniso }}^{\alpha}=\left(\begin{array}{ccc}
-a^{\alpha} & 0 & 0 \\
0 & 0 & 0 \\
0 & 0 & +a^{\alpha}
\end{array}\right)
$$

where the positive principal value is found along the $\mathrm{H}_{\alpha}-\mathrm{C}_{\alpha}$ bond axis and the 0 principal value indicates the direction parallel to the carbon lone electron $2 p_{z}$ orbital. Both $A_{i s o}^{\alpha}$ and $a^{\alpha}$ have been found to be proportional to the spin density on the $\mathrm{C}_{\alpha}$ atom $\rho^{\pi}$

$$
A_{\text {iso }}^{\alpha}=\rho^{\pi} Q_{i s o}^{\alpha} \quad a^{\alpha}=\rho^{\pi} Q_{\text {aniso }}^{\alpha}
$$

known as the McConnell [75] and Gordy-Bernhard [68] relations, respectively. Common values are $Q_{i s o}^{\alpha}=-72 \mathrm{MHz}$ and $Q_{\text {aniso }}^{\alpha}=38.7 \mathrm{MHz}$, and from actually measured $\alpha$-proton HF couplings the spin density on the $\mathrm{C}_{\alpha}$ can be estimated.

(a)

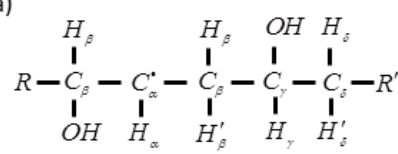<smiles>[R]C([R])([2H])[2H]</smiles>

(c)<smiles></smiles>

Fig. 6.5. Radical fragments that may be expected in carbohydrates. (a) pure hydroxyalkyl radical (b) alkoxy radical (c) hydroxyalkyl radical with a carbonyl group in a $\beta$ position. 
The isotropic HF coupling of a $\beta$-proton, due to hyperconjugation, is given by the Heller-McConnell relation [76]

$$
A_{i s o}^{\beta}=\rho^{\pi}\left(B_{0}+B_{2} \cos ^{2} \theta\right)
$$

where $\theta$ is the dihedral angle between the $p_{z}$ lone electron orbital axis and the $\mathrm{C}_{\beta}-$ $\mathrm{H}_{\beta}$ bond, viewed along the $\mathrm{C}_{\alpha}-\mathrm{C}_{\beta}$ bond. $B_{0}$ and $B_{2}$ are empirical constants. $B_{0}$ is often assumed to be close to $0 \mathrm{MHz}$ and for alkyl, hydroxyalkyl and alkoxy radicals typical $B_{2}$ values of $\sim 126, \sim 73$ and $\sim 336 \mathrm{MHz}$ are commonly taken $[77,78]$. Based on estimations for the spin density, relation (6.6) is regularly used to estimate dihedral angles for $\beta$-protons. The isotropic HF couplings for protons in $\gamma, \delta$, etc. positions are in general considerably smaller than those expected for $\beta$ protons. For all these proton couplings, the anisotropic HF interaction is dominated by the point dipole contribution and is therefore essentially axial around the $\mathrm{H} \cdots \mathrm{C}_{\alpha}$ axis [49]

$$
\vec{A}_{\text {aniso }}^{\beta, \gamma, \delta}=\left(\begin{array}{ccc}
-b & 0 & 0 \\
0 & -b & 0 \\
0 & 0 & +2 b
\end{array}\right)
$$

with, in frequency units $(\mathrm{MHz})$

$$
b=\frac{\mu_{0}}{4 \pi h} \frac{\left(g_{e} \mu_{B}\right)\left(g_{N} \mu_{N}\right)}{R^{3}} \rho^{\pi}
$$

where $\mu_{0}$ is the vacuum permeability and $R$ the distance between the $\mathrm{C}_{\alpha}$ carbon and the interacting $\mathrm{H}$. For $\beta$-protons, the deviation from these axial formula may be considerable and an appreciable rhombicity is often found (principal values in Eq. (6.7) being $-b-d,-b+d$, and $2 b$ ). For C-bound protons further away the pointdipole approximation works quite well and allows estimating $\mathrm{H} \cdots \mathrm{C}_{\alpha}$ distances and directions [79]. Comparison of these distances and directions with values for the intact molecule in the undamaged lattice, along with the number of $\alpha$ - and $\beta$-type interactions observed, usually strongly limits the number of possible locations for the radical center. It is also interesting to note that hydroxyl $\beta$-proton HF tensors usually exhibit a larger anisotropic and a smaller isotropic part than those of Cbound protons in a similar position [80]. Studies on crystals grown from $\mathrm{D}_{2} \mathrm{O}$ solutions, where the exchangeable oxygen-bound protons have been replaced by deuterium, are of course the most effective way for distinguishing hydroxyl from carbon-bound proton interactions.

${ }^{13} \mathrm{C}$ isotopic substitution is also a very powerful, but expensive, tool for identifying the center of spin density of radicals. It is hardly affordable to grow single 
crystals $100 \%$ enriched in ${ }^{13} \mathrm{C}$ for angular dependent EPR and ENDOR studies, but for systems in which the radicals have been thoroughly characterized on crystals with natural $\mathrm{C}$ isotopic distribution, powder EPR (if possible complemented with powder ENDOR) measurements may provide unambiguous information [13, 81].

\subsubsection{Semi-empirical theory: information obtained from g tensors}

C- and O-centered radicals may easily be distinguished from each other via their $g$ tensors. As typical example of $g$ tensor for a $\mathrm{C}$-centered radical without spin delocalization on oxygen, one can take the radical in malonic acid, for which $g_{x}=2.0026, g_{y}=2.0033$ and $g_{z}=2.0035$ have been measured with EPR [82] and $g_{x}=2.0023, g_{y}=2.0033$ and $g_{z}=2.0037$, using EIE [62]. The smallest principal value, which nearly coincides with the free electron value $g_{e}$, marks the direction of the lone-electron orbital. For the other principal values only small positive shifts $\Delta g=0.0010-0.0020$ are expected [83] and observed. For pure alkyl and hydroxyalkyl radicals (Fig. 6.5a), with a high spin density at the radical C-center, similar $g$ tensors are expected.

Alkoxy radicals, on the other hand, obtained by $\mathrm{H}$-abstraction from a sugar $\mathrm{OH}$ group (Fig. 6.5b), have their spin density mainly localized on an oxygen atom. They exhibit a more pronounced $g$ anisotropy mainly due to the much larger spinorbit coupling constant of $\mathrm{O}$ with respect to that of $\mathrm{C}[15,84]$. They are easily recognized in angular dependent EPR spectra, because their transitions move strongly with the magnetic field orientation. At certain orientations their resonances move far outside the spectral range of the (usually more prominent) C-centered radicals, whose spectral center hardly moves when the magnetic field is rotated (at least at $\mathrm{X}$-band) and whose range and anisotropy are dominated by HF splitting. For alkoxy radicals $1.970<g_{x}<2.0023 ; 2.005<g_{y}<2.009$ and $2.021<g_{z}<2.11$ have been reported [84-88]. Again, the smallest $g$ value (closest to $g_{e}$ ) indicates the loneelectron orbital direction, while the largest $g$ value is found along the $\mathrm{C}-\mathrm{O} \bullet$ bond.

C-centered radicals have been reported with considerably larger $g$ shifts (and anisotropy) than simple alkyl and hydroxyalkyl radicals. The $\mathrm{T} 1 \mathrm{radical}$ with principal $g$ values presented in Table 6.2 is an example of this type, $\mathrm{R}-(\mathrm{C}=\mathrm{O}){ }^{-} \mathrm{C}-$ R'R". These enhanced $g$ shifts are attributed to spin delocalization onto oxygen: ring oxygen and/or $\mathrm{C}=\mathrm{O}$ (carbonyl) groups close to the $\mathrm{C}_{\alpha}$, usually in $\beta$ positions (resonance contribution of structures like R-(C-O')=C-R'R', see Fig. 6.5c). $g_{x}$, found along the lone-electron orbital, remains close to $g_{e}$, but $g_{y}$ and $g_{z}$ undergo larger positive shifts. In the case of spin delocalization onto a $\beta$-carbonyl, $g_{z}$ is found (close to) parallel with the $\mathrm{C}=\mathrm{O}$ bond axis, often in the range 2.00552.0080 . 


\subsubsection{DFT calculations : evolving methodology}

Very early in our research of radiation-induced radicals in nucleic acid constituents, amino acids and carbohydrates, it was realized that isotropic HF couplings alone, as one would determine from solution or powder EPR experiments, are not sufficiently discriminative with respect to the radical model. Angular dependent ENDOR experiments provide detailed information about anisotropy of the HF interactions, and this information should also be fully exploited in the comparison with DFT calculations of SH parameters. The DFT modeling of $g$ and HF tensors generally proceeds in two steps. First a geometry optimization is performed and next the SH parameters are calculated for the optimized radical geometry. The reliability of the results depends critically on how accurately the environment of the radical is taken into account in these two steps.

In our very first computational studies on radicals in carbohydrates, only single molecule calculations were computationally feasible [89]. For the geometry optimization this implied that constraints needed to be imposed and that only part of the molecular radical was allowed to relax. For taking the directional information into account, angles between principal directions of the various experimental HF tensors were compared with the DFT calculated counterparts. Such comparisons later on proved insufficient for credible radical model assignments. In a better approach, the orientation of the molecule in the crystal was properly taken into account, which allowed more complete comparisons between experimental and calculated HF tensors and already proved quite successful in radical model identification $[10,90]$.

An obvious improvement consisted in embedding of the molecular radical in neighboring intact molecules for the geometry optimization, while the HF tensor calculations still were performed on single molecules [91]. This allowed for more realistic atomic relaxations around the radical center. In the subsequent step in the modeling refinement, the embedding was also applied for the tensor calculations $[16,92]$. The molecules hydrogen-bound close to the radical center appeared to have the largest influence, both on the geometry and on the calculated HF parameters. In all geometry optimizations, Gaussian type orbitals and gradient corrected exchange correlation functionals were employed; for calculations of $\mathrm{HF}$ and $g$ tensors, hybrid functionals were often used.

The most natural way of taking the crystalline environment of the radical into account is, however, via periodic boundary conditions. A potential draw-back of this method is that interactions between the periodic images of the radical have to be avoided. This may be accomplished by taking a unit cell in the periodic calculations which is larger than the crystallographic unit cell (the supercell approach). In the past five years in all our studies the geometry optimizations were performed in periodic schemes based on Car-Parrinello Molecular Dynamics using plane wave and pseudopotential basis sets (CPMD [93, 94]) and variants employing 
Gaussian and plane wave, and Gaussian and augmented plane wave basis sets (CP2K [95-97]). Supercells doubled or quadrupled in size with respect to the crystallographic unit cell along the shortest lattice parameters proved sufficient, although this choice still implies a high radical concentration of 5-25\%. It was verified that periodic cell sizes larger than about $1 \mathrm{~nm}$ along each crystal axis do no longer demonstrate influences of interactions between radicals in periodic images (see e.g. [8]). As opposed to single molecule and cluster calculations, no constraints on atomic relaxations have to be imposed in this type of calculations.

In these first attempts to properly take into account the full lattice environment of the radical, SH parameter calculations were still performed on clusters cut out of the periodically optimized structure, because $g$ and HF tensors reflect local properties, which are known not to be well reproduced by plane wave basis sets $[8,9,14,17-19,55]$. A further major improvement in the calculations came from the implementation of HF [98] and $g$ tensor calculations [99, 100] in periodic codes $(\mathrm{CP} 2 \mathrm{~K})$ using Gaussian and augmented plane wave basis sets. This allowed to perform the SH parameter calculations directly on the periodically optimized structure. Our latest computational results for neutral carbohydrate radicals are all performed in this way $[12,20,21,57,101]$. In the case of charged radicals, the periodic boundary conditions induce an infinite charge on the lattice, which would have to be balanced by a background countercharge density.

A common problem in this context - not only for the periodic boundary calculations, but also for cluster modeling - is that of localization of charge and spin. Adding or subtracting an electron to/from a model system, mimicking the formation of a reduced or an oxidized radical species, quite often results in the charge and spin becoming distributed over several molecules, or even over the entire system. There are not many remedies to this problem. Possibly the implementation and use of other functionals, containing a higher degree of exact Hartree-Fock exchange, might be helpful [102]. Sometimes, it is sufficient to make slight changes to the geometry of one molecule in the lattice to make the spin (and/or charge) localized at that particular molecule.

\subsubsection{Practical example of radical identification}

We return to the example of the T1 stable radical in irradiated sucrose, whose $g$ (see Table 6.2) and proton $A$ tensors (see Table 6.3) have already been discussed in the context of Schonland ambiguity (Section 6.2.4). This ambiguity has been solved for all tensors, however, and we only consider the first tensor in Table 6.2 and the $1^{\text {st }}, 3^{\text {rd }}$ and $5^{\text {th }}$ tensor in Table 6.3 for semi-empirical theoretical analysis and comparison with DFT calculations. Experiments on crystals grown from $\mathrm{D}_{2} \mathrm{O}$ solutions yielded the same three HF couplings, demonstrating that none of the $A$ tensors correspond to $\mathrm{OH}$ proton couplings. The main purpose here is to illustrate 
the principles of identification based on comparison between experimental and DFT computed SH parameters, for models constructed using the semi-empirical theoretical considerations discussed in Sections 6.3.1 and 6.3.2. A more elaborate discussion is found in Ref. [9].

The negative isotropic HF value and the rather large and fairly axial anisotropic interaction, make HF3 quite atypical and for that reason less straightforward to be used in a semi-empirical analysis. The tensors HF1 and HF2, on the other hand, exhibit a positive isotropic HF value and close to axial anisotropy. These properties are typical of regular $\beta$ - or $\gamma$-type couplings. From equations (6.7) and (6.8) the $\mathrm{H} \cdots \mathrm{C}$ distances between the radical's central carbon and the interacting proton are estimated at $2.0 \AA$ and $2.2 \AA$ for HF1 and HF2, respectively. These values are close to those expected for protons in $\beta$-positions (2.1-2.2 $\AA$ ). The weaker HF2 interaction could, however, also come from a $\gamma$-proton whose coupling is atypically large because it is next to a ring oxygen or a carbonyl group, onto which a considerable amount of spin density is delocalized. The enhanced $g$ tensor anisotropy for this radical, indeed, suggests a significant spin delocalization onto oxygen for this center.

In order to narrow down the possibilities for the location of the radical center, the $\mathrm{C} \cdots \mathrm{H}$ directions in the intact sucrose molecule (in the crystal) are compared with the principal directions corresponding to the largest principal $A$ value for HF1 and HF2 for both symmetry-related sites. The smallest deviation angles $\delta$ are listed in Table 6.4. The comparison yields $\mathrm{C} 1, \mathrm{C} 4$ and $\mathrm{C} 2$ ' as possible radical centers, with similar deviations in the order of $20^{\circ}$.

Table 6.4. Atomic distance $\mathrm{D}$ (in $\AA$ ) and angle $\delta$ (in degrees) between $\mathrm{C}-\mathrm{H}$ directions (see Table 1 and Fig. 6.6a for atom numbering) in the crystalline sucrose molecule and principal directions of the largest principal value for the HF1 and HF2 A tensors (Table 6.3) [9]

\begin{tabular}{cccccccc}
\hline Direction & H-position & $\mathrm{D}$ & $\langle a *\rangle$ & $\langle b\rangle$ & $\langle c\rangle$ & $\delta(\mathrm{HF} 1)$ & $\delta(\mathrm{HF} 2)$ \\
\hline C1-H2 & $\beta$ & 2.14 & 0.556 & -0.454 & 0.696 & 21.3 & \\
C1-H5 & $\gamma$ & 2.74 & -0.139 & 0.647 & -0.750 & & 15.7 \\
& $\beta$ & 2.15 & 0.542 & 0.443 & 0.714 & 21.6 & \\
C4-H5 & $\beta$ & 2.69 & -0.148 & 0.652 & -0.744 & & 16.4 \\
C4-H2 & $\gamma$ & & & & & & \\
C2'-H3' & $\beta$ & 2.16 & 0.854 & 0.420 & 0.307 & 21.7 & \\
C2'-H5' & $\gamma$ & 3.01 & 0.077 & 0.731 & -0.678 & & 13.9 \\
\hline
\end{tabular}

In Fig. 6.6, the intact sucrose molecule is presented along with five radical models that can be obtained at the three sites found plausible for T1, and for which spin density delocalization onto neighboring oxygen atoms is expected. The first four models $\left(\mathrm{M}(\mathrm{C} 1), \mathrm{M}(\mathrm{C} 4), \mathrm{M}\left(\mathrm{C} 2^{\prime}\right) \mathrm{a}\right.$ and $\left.\mathrm{M}\left(\mathrm{C} 2^{\prime}\right) \mathrm{b}\right)$ are the simplest radicals one can obtain at these positions with the given specifications, the fifth $\left(\mathrm{M}\left(\mathrm{C} 2^{\prime}\right) \mathrm{c}\right)$ is a further modification of the fourth model $\left(\mathrm{M}\left(\mathrm{C} 2^{\prime}\right) \mathrm{b}\right)$. An overview of the computed 
principal HF values for these models is given in Table 6.5, along with the differences in principal values and directions with the HF1 and HF2 tensors. The couplings with only small and rather isotropic HF values, expected to contribute mainly to the ${ }^{1} \mathrm{H}$ matrix ENDOR line, are omitted. For the angles between principal directions, both symmetry-related sites of the experimental tensor are considered and the results are presented for the site yielding the smallest angles. The radical geometry was optimized in a periodic scheme using a supercell consisting of two crystallographic unit cells along the $\langle c\rangle$ direction. HF tensor calculations were performed in a single molecule approach (radical molecule cut out from the periodically optimized structure) using Gaussian03, with a B3LYP hybrid functional and a 6-311G(d,p) basis set.
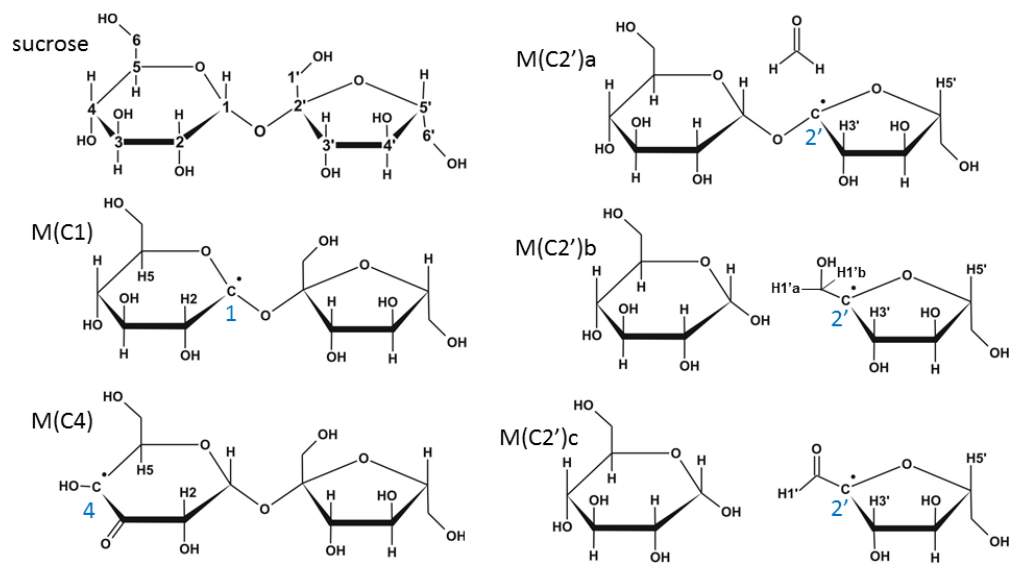

Fig. 6.6. Intact sucrose molecule, defining the labeling of the carbon atoms, and radical models for $\mathrm{T} 1$ in sucrose, compatible with the location determined by comparison of the principal directions of tensors HF1 and HF2 (Table 6.3) with crystal directions (Table 6.4) and with the observed large $g$-shifts (considerable spin delocalization onto oxygen) [9]. The central carbon of the radicals and the hydrogen nuclei exhibiting appreciable HF interaction (DFT calculated values in Table 6.5) are numbered.

$\mathrm{M}(\mathrm{C} 1)$ is obtained by simple hydrogen abstraction at the $\mathrm{C} 1$ atom. For neither of the HF interactions, the isotropic and anisotropic HF values seem in good agreement with experiment. The principal directions of the $\beta$-proton appear to be in fair agreement with those of HF1 (deviations smaller than $20^{\circ}$ ), but for the $\gamma$ proton a more severe discrepancy is observed. In addition, this model does not account for the third HF coupling experimentally observed.

$\mathrm{M}(\mathrm{C} 4)$ is obtained by hydrogen abstraction at $\mathrm{C} 4$ and carbonyl group formation at $\mathrm{C} 3$. This model does yield three HF tensors with isotropic couplings in the right order of magnitude, one having a negative isotropic value, as observed experimentally. The latter interaction, however, is due to a hydroxyl proton, in contradiction with the experiments on deuterated crystals. In addition, the principal directions of 
the calculated HF tensors deviate very strongly from those of HF1 and HF2. Hence, also his model should not be further considered.

Model $\mathrm{M}(\mathrm{C} 2$ ') $)$ a is obtained by splitting off a formaldehyde molecule at $\mathrm{C} 2$ '. Additional constraints had to be imposed in the periodic geometry optimization in order to prevent spontaneous reattachment of the formaldehyde and formation of the $\mathrm{O} 1$ '-centered alkoxy radical. In the HF tensor calculations, the formaldehyde molecule was left out. The model again yields only two appreciable HF couplings: one $\beta$-coupling and one $\gamma$-coupling with negative $A_{\text {iso. }}$. The very strong deviations in principal directions with the experimental tensors suggest that also this model may be eliminated.

$\mathrm{M}\left(\mathrm{C}^{\prime}{ }^{\prime}\right) \mathrm{b}$ is obtained by cleaving the glycosidic bond in the sucrose molecule at the fructose side. The calculations yield three considerable HF couplings. For two of them, the anisotropy and principal directions are in acceptable agreement with HF1 and HF2, but the deviation in $A_{\text {iso }}$ for both tensors is quite large. In particular, $A_{\text {iso }}$ for the interaction with the $\beta$-proton H3' is strongly overestimated. The most important flaw of the model is, however, the large $\beta$-proton interaction with H1'a, not observed in experiment. A modification of this model eliminates these two problems: carbonyl formation (by $\mathrm{H}_{2}$ elimination) on the $\mathrm{C} 1$ ' position is expected to decrease the spin density on the central C2' atom and hence also $A_{\text {iso }}$ for the H3' proton. At the same time, the remaining $\mathrm{H} 1{ }^{\prime}$ is expected to lie in the ${ }^{\circ} \mathrm{C} 2-\mathrm{C} 1=\mathrm{O}$ plane (perpendicular to the lone electron $p_{z}$ orbital), which should, according to equation (6.6), result in a relatively small HF coupling. The results of the HF tensor calculations on this model, labeled $\mathrm{M}\left(\mathrm{C} 2{ }^{\prime}\right) \mathrm{c}$ in Fig. 6.6, even in this rather simple computational scheme, lead to very good quantitative agreement with experiment for HF1 and HF2. Moreover, the HF interaction with the remaining H1' proton appears to exhibit the characteristics of HF3. Improvements of the calculation scheme lead to an even better agreement, especially for the latter coupling.

In Table 6.6 the results of HF and $g$ tensor calculations on the periodically optimized model $\mathrm{M}\left(\mathrm{C} 2^{\prime}\right) \mathrm{c}$ are shown, using a supercell comprising 8 crystallographic unit cells. The HF tensor calculations, which came earlier [9], were performed on a cluster containing the radical molecule and 10 sucrose molecules hydrogenbound to it. The more recent $g$ tensor calculations [57] are all-periodic. The agreement between the four experimental tensors and their calculated counterparts is very good on all accounts. In particular we want to emphasize here that the agreement in principal directions for $g$ and $A$ tensors is excellent. It is also important to note that all other HF couplings calculated for this model are much smaller than those listed in Table 6.3. These are expected to contribute in the ENDOR spectra to the multitude of ENDOR lines near the ${ }^{1} \mathrm{H}$ Larmor frequency and to be hidden in the EPR line width. 
Table 6.5. Calculated principal HF values (MHz) for five radical models for T1 (Fig. 6.6) and deviations in values and directions ( $\delta$, in degrees) with HF1 and HF2 (see Table 6.3) [9]

\begin{tabular}{|c|c|c|c|c|c|c|}
\hline Model & Proton & $A_{\text {iso }}$ & $\Delta A_{\text {iso }}$ & $A_{\text {aniso }, i}$ & $\Delta A_{\text {aniso }, i}$ & $\delta$ \\
\hline \multirow[t]{6}{*}{$\mathrm{M}(\mathrm{C} 1)$} & & & & -6.08 & -2.09 & 15.9 \\
\hline & $\beta-\mathrm{H} 2$ & 32.85 & -13.95 & -4.78 & -2.40 & 12.4 \\
\hline & & & & 10.86 & 4.49 & 19.8 \\
\hline & & & & -4.44 & -1.75 & 23.3 \\
\hline & $\gamma-\mathrm{H} 5$ & -2.60 & -18.48 & -1.33 & 0.98 & 29.0 \\
\hline & & & & 5.77 & 0.77 & 24.2 \\
\hline \multirow[t]{9}{*}{$\mathrm{M}(\mathrm{C} 4)$} & & & & -3.65 & 0.34 & 43.4 \\
\hline & $\beta-\mathrm{H} 5$ & 56.60 & 9.8 & -2.62 & -0.24 & 44.7 \\
\hline & & & & 6.27 & -0.10 & 34.2 \\
\hline & & & & -2.65 & 0.04 & 56.3 \\
\hline & $\gamma-\mathrm{H} 2$ & 10.86 & -5.02 & -1.40 & 0.91 & 64.0 \\
\hline & & & & 4.05 & -0.95 & 33.3 \\
\hline & & & & -8.38 & & \\
\hline & $\mathrm{HO} 4$ & -10.12 & & -7.37 & & \\
\hline & & & & 15.74 & & \\
\hline \multirow[t]{6}{*}{$\mathrm{M}\left(\mathrm{C} 2^{\prime}\right) \mathrm{a}$} & & & & -5.82 & -1.83 & 63.2 \\
\hline & $\beta-\mathrm{H} 3$ & 32.15 & -14.65 & -5.23 & -2.85 & 67.7 \\
\hline & & & & 11.05 & 4.68 & 21.7 \\
\hline & & & & -3.31 & -0.62 & 67.8 \\
\hline & $\gamma-\mathrm{H} 5$, & -3.01 & -18.89 & -2.29 & 0.02 & 69.9 \\
\hline & & & & 5.60 & 0.60 & 22.8 \\
\hline \multirow[t]{9}{*}{$\mathrm{M}\left(\mathrm{C} 2^{\prime}\right) \mathrm{b}$} & & & & -5.22 & -1.23 & 16.3 \\
\hline & $\beta-\mathrm{H} 3$, & 66.83 & 20.03 & -2.70 & -0.32 & 17.3 \\
\hline & & & & 7.91 & 1.55 & 6.8 \\
\hline & & & & -4.24 & -1.55 & 6.1 \\
\hline & $\gamma-\mathrm{H} 5$, & 6.95 & -8.93 & -2.90 & -0.59 & 11.7 \\
\hline & & & & 7.14 & 2.14 & 10.3 \\
\hline & & & & -5.96 & & \\
\hline & $\beta-\mathrm{H} 1$ 'a & 56.66 & & -2.77 & & \\
\hline & & & & 8.73 & & \\
\hline \multirow[t]{9}{*}{$\mathrm{M}\left(\mathrm{C} 2^{\prime}\right) \mathrm{c}$} & & & & -3.79 & 0.20 & 3.9 \\
\hline & $\beta-\mathrm{H} 3$, & 44.40 & -2.20 & -2.52 & -0.14 & 4.1 \\
\hline & & & & 6.31 & -0.06 & 1.5 \\
\hline & & & & -2.60 & 0.09 & 8.0 \\
\hline & $\gamma-\mathrm{H} 5$ & 12.90 & -2.98 & -2.20 & 0.11 & 8.2 \\
\hline & & & & 4.80 & -0.20 & 2.5 \\
\hline & & & & -5.08 & & \\
\hline & $\beta-\mathrm{H} 1^{\prime}$ & -6.50 & & -3.30 & & \\
\hline & & & & 8.37 & & \\
\hline
\end{tabular}


To further exemplify the issue about Schonland conjugates and radical identification, in the final three columns of Table 6.6 the DFT calculated tensors are also compared with the Schonland conjugates of the experimental tensors. It is clear that Schonland ambiguity may blur or even hamper the identification of radicals based on DFT calculations of SH parameters. Not surprisingly, the differences between DFT calculated and Schonland conjugate experimental tensors are largest for HF3 and for the $g$ tensor for which also the largest differences between the Schonland conjugate forms of the experimental tensors were found. Even having only one tensor in the Schonland conjugate form would render the identification considerably less convincing.

Table 6.6. DFT calculated proton principal HF (in $\mathrm{MHz}$ [9]) and $g$ [57] values for the $\mathrm{M}(\mathrm{C} 2$ ') model for T1, depicted in Fig. 6.6, and deviations in values ( $\Delta g$ values in parts per thousand) and directions $(\delta$, in degrees) with experimental data for this radical (Tables 6.2 and 6.3). In order to illustrate the problem of Schonland ambiguity, the final three columns give the differences in principal values and directions with the Schonland conjugate tensors, although in this particular case the ambiguity was solved by measurements in a fourth rotation plane [9]

\begin{tabular}{|c|c|c|c|c|c|c|c|c|}
\hline Proton & $A_{\text {iso }}$ & $A_{\text {aniso,i }}$ & $\Delta A_{\text {iso }}$ & $\Delta A_{\text {aniso,i }}$ & $\delta$ & $\Delta A_{\text {iso }}$ & $\Delta A_{\text {aniso }, i}$ & $\delta$ \\
\hline \multirow{3}{*}{$\beta-\mathrm{H} 3$} & \multirow{3}{*}{41.90} & -3.74 & \multirow{3}{*}{-4.90} & 0.25 & 4.7 & \multirow{3}{*}{-4.90} & 1.16 & 18.5 \\
\hline & & -2.33 & & 0.05 & 4.7 & & -1.10 & 19.2 \\
\hline & & 6.07 & & -0.30 & 1.1 & & -0.06 & 7.1 \\
\hline \multirow{3}{*}{$\gamma-\mathrm{H} 5$} & \multirow{3}{*}{16.30} & -2.40 & \multirow{3}{*}{0.42} & 0.29 & 9.0 & \multirow{3}{*}{0.43} & 0.81 & 21.3 \\
\hline & & -2.26 & & 0.05 & 9.2 & & -0.54 & 21.8 \\
\hline & & 4.67 & & -0.33 & 1.6 & & -0.27 & 4.5 \\
\hline \multirow{3}{*}{$\beta-\mathrm{H} 1$} & \multirow{3}{*}{-11.47} & -6.92 & \multirow{3}{*}{-0.40} & -0.51 & 5.9 & \multirow{3}{*}{-0.35} & 1.07 & 22.7 \\
\hline & & -4.64 & & -0.91 & 5.8 & & -3.69 & 23.7 \\
\hline & & 11.56 & & 1.42 & 4.6 & & 2.62 & 13.5 \\
\hline \multirow{3}{*}{$g$} & \multirow{3}{*}{2.0043} & -0.0020 & \multirow{3}{*}{-0.21} & 0.2 & 7.4 & \multirow{3}{*}{-0.22} & 0.3 & 14.7 \\
\hline & & 0.0003 & & -0.3 & 7.3 & & -0.5 & 21.1 \\
\hline & & 0.0018 & & -0.5 & 1.4 & & -0.4 & 25.8 \\
\hline
\end{tabular}

The results presented here illustrate the importance of an accurate $\mathrm{SH}$ analysis of experimental data: having the right model at hand and performing calculations of sufficiently high level (taking the molecular environment into account in a correct way), the agreement between experiment and calculations may be impressive. Our experience tells us that in convincing identifications, for large isotropic HF values (in particular $\alpha$-protons) deviations of $10-15 \mathrm{MHz}$ may still occur, though. For anisotropic $g$ and $A$ values, however, deviations should not be larger than 10 $20 \%$. All SH tensors should have at least one principal direction (and very often all) for which the deviation between experiment and calculation is well below $10^{\circ}$. Furthermore, all calculated HF couplings of significant magnitude $(>10-20 \mathrm{MHz})$ should have an experimental counterpart, either determined from ENDOR angular 
variation results or inferred from the EPR/EIE spectra, when the corresponding ENDOR transitions are experimentally not detected.

In the following sections, explicit comparisons between experimental and DFT calculated $g$ and HF tensors will be limited. Based on the comparison criteria set out above, we will label identifications as "certain" if all criteria are met, comparable to the situation for T1. Structures are labeled "plausible" if for certain tensors the criteria are not fully met, and "suggested" if only qualitative agreement is obtained, e.g. because certain experimental couplings are not predicted by the calculations or vice versa. The label "proposed" is used when models are derived from experimental studies only, without explicit verification by DFT calculations.

\subsection{Radical stability and evolution vs. radiation chemistry}

In this section, we review the results of radical identification studies on sucrose after irradiation at various temperatures, immediately after irradiation or after annealing to temperatures higher than the irradiation temperature. Irradiation and annealing temperatures range between $\sim 6-8 \mathrm{~K}$ and RT. The ultimate aim of such studies is to unravel the radiation chemistry that leads to the production of the final stable radicals. The structures of these radicals (see e.g. T1, Section 6.3.4) suggest complex, multistep formation mechanisms, wherein the primary ionization or electron capture event is followed by various thermally activated structural relaxations and chemical reactions. At lower temperatures, (some of) these subsequent reactions may be frozen out, leaving the radical in a metastable state that is closer to its primary form. As large structural rearrangements are not expected at low temperatures, these metastable radicals often are the result of breaking of a bond or abstraction of a small fragment in the molecule $(\mathrm{H}, \mathrm{OH})$. Adequate structures are in general more easily found than for radicals with higher thermal stability. Knowledge of the structure of these (close to) primary radicals, and understanding why exactly these radicals and not others are produced, is important in the context of understanding and/or controlling the (initial) radiation damage to more complex biomolecules, like DNA. In addition, following the subsequent transformations upon thermal annealing may help in unraveling the structure of the more complex RT stable radicals. We take sucrose as an example for explaining the methodology in this section, because for this carbohydrate the most comprehensive experimental data set is available. Moreover, it illustrates very well all pitfalls of this type of analyses. Some of the results presented in this Section have not been published before [56, 103-106]. In Section 6.5, the results for the sugar phosphate $\mathrm{K} 2 \mathrm{G} 1 \mathrm{P}$, the disaccharide trehalose dehydrate, and the monosaccharides fructose and sorbose are reviewed.

Before embarking on the discussion of the available experimental results, a remark about the methodology is in order. It is not a priori evident that low tem- 
perature irradiation followed by thermal annealing to RT is equivalent to RT irradiation in terms of radical products. In our studies of carbohydrates we have checked by monitoring EPR and ENDOR spectra, that the radical composition after low temperature irradiation and annealing to a certain temperature (up to RT) is the same as that after irradiation at the latter temperature: the same types of dominant radical species are present in approximately the same relative concentrations. This confirms that irradiation at low temperature and gradual anneal to RT may elucidate the complex radical formation processes at RT. The total EPR intensity after low temperature irradiation and annealing is, however, considerably lower than that after RT irradiation to similar doses. This suggests that radical recombination is more important in the former process than in the latter. It should further be born in mind that in most analyses (see further in Sections 6.4 and 6.5), radical components with low EPR (and ENDOR) intensities have remained unidentified. Furthermore and perhaps more importantly, EPR is only sensitive to the paramagnetic forms of radiation damage, not to diamagnetic radiation products.

\subsubsection{Types of annealing experiments}

In order to follow the radical evolution after the formation of the primary radiation products, one may decide to monitor changes in the EPR spectrum of crystals initially irradiated at low temperature and then annealed for a long time (typically $>10$ min.) to increasing temperatures, recording EPR spectra at the annealing temperature. The results of such experiments at X-band for two orientations of a sucrose single crystal, initially irradiated at $10 \mathrm{~K}$, are presented in Fig. 6.7. In addition to the temperature evolution, isothermal evolution after RT irradiation is also included in the top few spectra of both figure parts (arrow labeled "Time"). All spectra were normalized in height. These experiments indicate that

i) the primary and finally stable radicals are substantially different and transformation between these two states goes through various steps. The stages at $10 \mathrm{~K}$ $[19,104], 80 \mathrm{~K}[105]$ and RT (metastable $[51,106]$ and fully stable $[8,9,55-58]$ ) have been investigated in more detail and will be discussed in the following subsections.

ii) the component(s) dominating the spectrum at $10 \mathrm{~K}$ is (are) still present and fairly dominant in the $80 \mathrm{~K}$ spectrum.

iii) the component(s) dominating the spectrum immediately after RT irradiation is (are) very probably already seen in the spectra after annealing to $200 \mathrm{~K}$.

The interpretation of experiments, such as shown in Fig. 6.7, presents two complications. First, this type of experiments does not allow distinguishing irreversible from reversible temperature-induced spectrum transformations. Second, the combined thermal population and paramagnetic relaxation effects [49] render a 
direct comparison of intensities of spectra recorded at different temperatures difficult. Pulse annealing experiments in which all EPR spectra are recorded at the same temperature after annealing (usually during constant annealing times, i.e. isochronal annealing) to increasing temperatures, eliminate these problems.
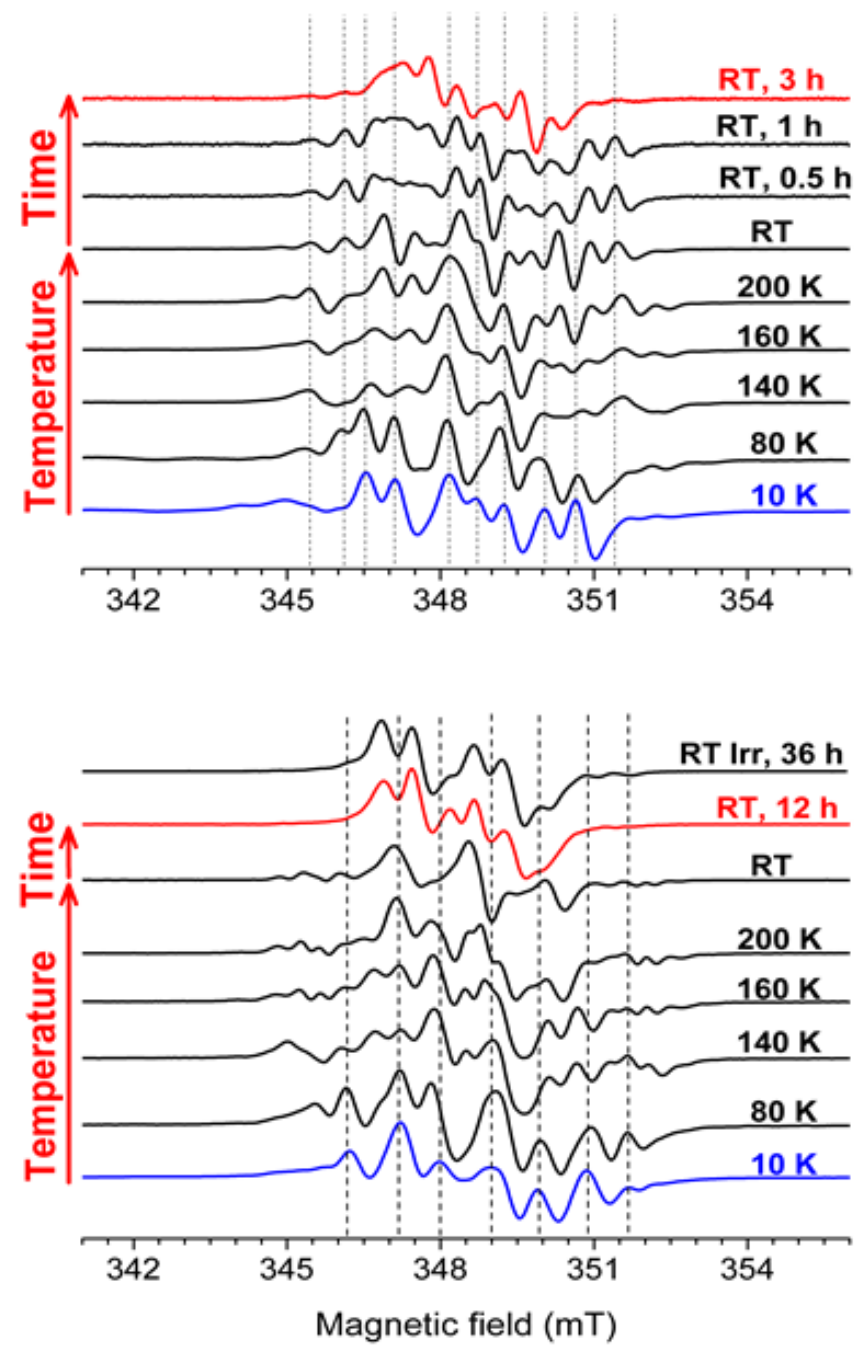

Fig. 6.7. EPR spectra of sucrose irradiated in situ at $10 \mathrm{~K}$ for $\mathrm{B} / /<b>$ (top) and $\mathrm{B} / /<c^{*}>$ (bottom) [103]. Annealing and recording temperatures are indicated on the right. The top spectrum with $\mathrm{B} / /\left\langle c^{*}>\right.$ was recorded at $\mathrm{RT}, 36 \mathrm{~h}$ after irradiation at RT. $v_{\mathrm{MW}}=9.78 \mathrm{GHz}$.

Their drawback is, however, that they are very time-consuming: each step comprises heating, stabilization and cooling times. Results of selected pulse annealing experiments are presented in Sections 6.4.2 and 6.4.6. For following the kinetics 
of radical transformations, isothermal annealing is most appropriate, in which EPR spectra are recorded at a constant temperature with fixed time intervals. Such experiments have so far only been performed sporadically in our studies of carbohydrates (see Section 6.4.4 and [51]).

\subsubsection{Close-to-primary radiation products (irradiation at 6-10 K)}

The X-band EPR and ENDOR spectra of sucrose recorded at $10 \mathrm{~K}$ after X-ray irradiation at $10 \mathrm{~K}$ contain contributions of at least six radical components, four of which dominate the first derivative EPR spectra [19]. Two of these were identified (certain) as $\mathrm{H}$-abstracted $\mathrm{C} 5$ - and $\mathrm{C} 1$-centered radicals in the glucose unit $(\mathrm{C} 5(-\mathrm{H})$ and $\mathrm{C} 1(-\mathrm{H})$, respectively). A third $\mathrm{C}$-centered radical was suggested to be produced by $\mathrm{H}$-abstraction at $\mathrm{C} 6(\mathrm{C} 6(-\mathrm{H}))$. Among all $\mathrm{H}$-abstracted radicals at $\mathrm{C}$ atoms, the $\mathrm{HF}$ tensors calculated for the $\mathrm{C} 6(-\mathrm{H})$ species matched the experimental tensors best. Still, considerable discrepancies, both in principal values and directions, were found for the two resolved HF interactions observed, whereas a third interaction predicted by the calculations was not found in experiment. The fourth identified (certain) species $\left(\mathrm{O}^{\prime}{ }^{\prime}\left(-\mathrm{e}^{-}\right)\right)$is $\mathrm{O}$-centered and was previously studied by Box and Budzinski [107]. Excellent agreement between experimental and calculated HF tensors was found for six proton interactions. The calculations explicitly confirmed the radical model that Box and Budzinski had proposed for this O3' centered alkoxy radical in the fructose unit, where the HO3' proton has migrated to the O4' oxygen of a neighboring molecule. The DFT calculations, however, showed that some of the HF tensor assignments made by Box and Budzinski needed correction. The models for these four radical species are shown in Fig. 6.8.
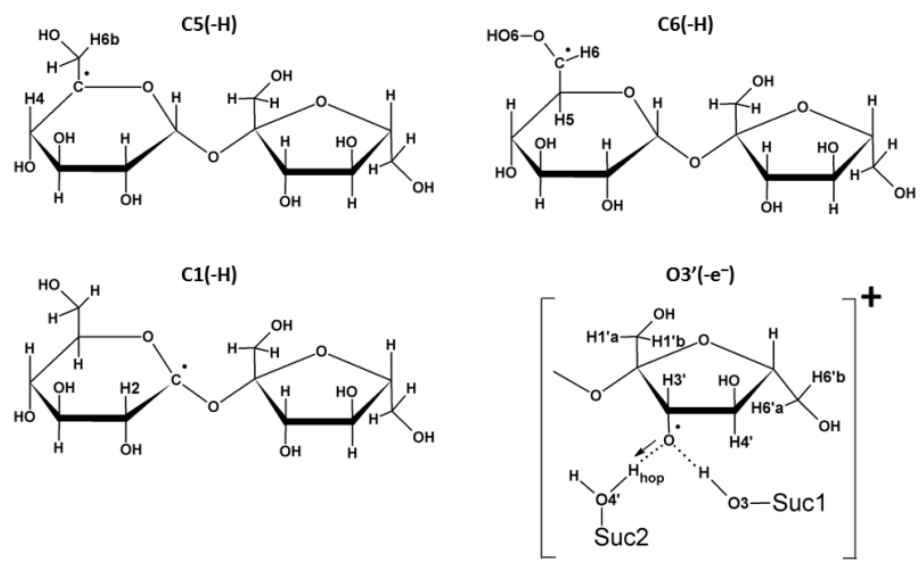

Fig. 6.8. Models for the four radical species dominating the EPR spectra of sucrose after X-ray irradiation at $10 \mathrm{~K}$. Protons with significant calculated HF interactions are labeled in the models [19]. 
Two minor radicals remained unidentified in the spectra, both are C-centered.

In previous investigations of sucrose irradiated at liquid helium temperature, an inter-molecularly trapped electron (IMTE) center had been detected and found to be stable in the dark up to $\sim 60 \mathrm{~K}[108,109]$. Like in other carbohydrates, this IMTE was characterized by large and anisotropic HF interactions with exchangeable protons and by a nearly isotropic $g$ value, slightly smaller than the freeelectron value. In the $\mathrm{X}$-band spectra after $10 \mathrm{~K}$ irradiation, this type of radical was not detected, neither with EPR nor with ENDOR. K-band (24 GHz) ENDOR experiments after $6 \mathrm{~K} \mathrm{X}$-ray irradiation, however, did reveal the presence of this IMTE [104].

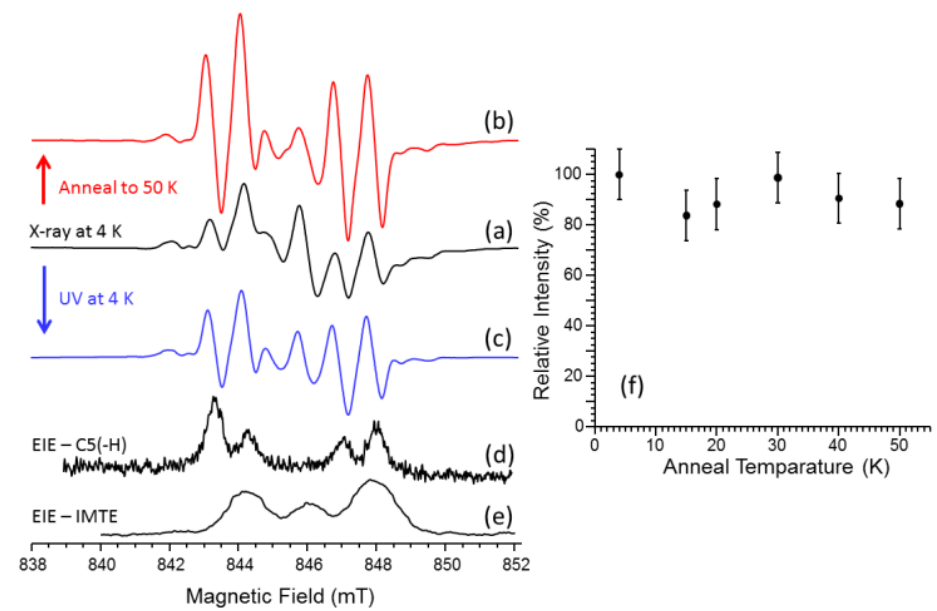

Fig. 6.9. K-band spectra $\left(v_{\mathrm{MW}}=23.69 \mathrm{GHz}\right)$ recorded at $6 \mathrm{~K}$ with $\mathrm{B} / /<b>$ of (a) a sucrose single crystal irradiated at $4 \mathrm{~K}$, (b) after irradiation at $6 \mathrm{~K}$ and subsequent thermal anneal to $50 \mathrm{~K}$, (c) after irradiation at $4 \mathrm{~K}$ and UV-bleaching at $6 \mathrm{~K}$. EIE spectra of (d) the $\mathrm{C} 5(-\mathrm{H})$ radical and (e) the IMTE. (f) Total double integrated EPR intensity as a function of annealing temperature in the pulse annealing experiment (measurements at $6 \mathrm{~K}$ ) [104].

Figure 6.9 shows EPR spectra after irradiation at $6 \mathrm{~K}$ and subsequent UVbleaching at $6 \mathrm{~K}$ or after thermal pulse anneal to $50 \mathrm{~K}$ (up to $40 \mathrm{~K}$ the EPR and ENDOR spectra remained unchanged). Both treatments appear to have similar effects: while the total double integrated intensity of the spectrum remains nearly unchanged (inset in Fig. 6.9 for the pulse annealing experiments) the EPR transitions of the $\mathrm{C} 5(-\mathrm{H})$ radical (corresponding EIE spectrum in Fig. 6.9d), characterized by a four-line HF structure and already present immediately after irradiation, apparently increase strongly at the expense of a broad-line EPR component (EIE spectrum in Fig. 6.9e). The ENDOR and EIE spectra recorded for the broad-line EPR spectrum correspond to the IMTE as reported by Budzinski et al. [108]. In particular, the slightly lower $g$ value of the IMTE triplet contribution is clearly recognizable. The slight discrepancy in annealing temperature $(50 \mathrm{~K}$ in our exper- 
iments vs. $60 \mathrm{~K}$ according to Budzinski et al.) is most likely only instrumental. Spectrum simulations show that the broad-line IMTE component easily remains undetected in EPR, whereas its relaxation properties may be unfavorable for ENDOR at X-band.

Closer inspection of Fig. 6.9 reveals that the effects of optical and thermal bleaching are not identical, though. The intensity increase of the $\mathrm{C} 5(-\mathrm{H})$ radical appears larger after thermal anneal than after UV-illumination. ENDOR experiments reveal that in both cases the IMTE signal has completely bleached out, while the total spectrum intensity remains practically identical. However, in the ENDOR spectra of annealed samples the presumed $\mathrm{C} 6(-\mathrm{H})$ radical appears to be missing, while after UV-bleaching at $6 \mathrm{~K}$ it was clearly observed.

These results suggest a transformation of the IMTE into the C5-centered radical as main reason for the spectral changes. Molecular modeling of the IMTE is far from obvious, not in the least because it requires electron orbitals (basis sets) that are not centered on lattice atoms, which comes at strongly increased computational cost and requires extensive testing of basis sets. Consequently, it has so far not been possible to determine the location of the IMTE by direct comparison of calculated and experimental HF tensors. It may however be anticipated that the electron will be trapped in the dipolar field between at least two $\mathrm{OH}$-dipoles in carbohydrate crystals [108-112]. Plausible sites for inter-molecular electron trapping in carbohydrates have been calculated applying the semi-continuum potential model [110], and considerable rearrangements of $\mathrm{OH}$-dipoles, even at $4 \mathrm{~K}$, have been suggested. In the particular case of sucrose, the most plausible site for the observed IMTE was determined by analysis of the anisotropy of non-exchangeable proton interactions, observed with ENDOR [108]. This site is shown in Fig. 6.10. It is situated close to the C5 and C6 atoms in the glucose unit.

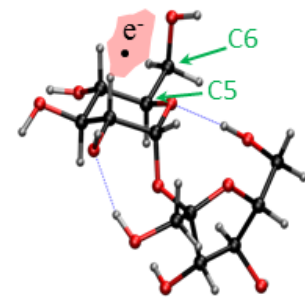

Fig. 6.10. Plausible site for the IMTE center in the sucrose lattice [108].

Considering this site assignment for the IMTE, the following scheme for the production of the $\mathrm{C} 5(-\mathrm{H})$ radical can be devised [19], in agreement with mechanisms proposed by Samskog et al. for trehalose [111] and rhamnose [112]

$$
\begin{array}{cccc}
\mathrm{R}-\mathrm{OH}+\mathrm{e}^{-} & \rightarrow[\mathrm{R}-\mathrm{OH}]^{-} \underset{\mathrm{R}-\mathrm{O}^{-}}{\rightarrow}+\mathrm{H}^{\cdot} \\
\mathrm{R}^{\prime}-\mathrm{CHOH}-\mathrm{R}^{\prime \prime} & +\mathrm{H}^{\cdot} \rightarrow \mathrm{R}^{\prime}-\mathrm{C}^{\cdot} \mathrm{OH}-\mathrm{R}^{\prime \prime}+\mathrm{H}_{2}
\end{array}
$$


A similar formation mechanism (with $\mathrm{R} "=\mathrm{H}$ ) could, in principle, also be valid for the radical assigned as C6(-H). The mechanism outlined in Eq. (6.9) in itself does not allow to identify the actual site for the initial electron trapping on the sucrose molecule. The fact that UV-illumination after X-ray irradiation increases the C5 $(-\mathrm{H})$ concentration appears to support the site assignation by Budzinski et al., although it seems strange then that UV-exposure does not lead to a similar increase of the C6(-H) concentration. The difference between UV-bleaching and thermal annealing experiments, on the other hand, might be a result of a thermally activated transformation of $\mathrm{C} 6(-\mathrm{H})$ to $\mathrm{C} 5(-\mathrm{H})$. It seems in any case very plausible that these two radical types are both reduction radiation products. The alkoxy radical, which still has the H3' proton in its vicinity, is most likely an oxidation product. EPR spectrum simulations allowed to estimate the relative contributions of the various radical types to the spectra : $\mathrm{C} 5(-\mathrm{H})-15 \%, \mathrm{C} 1(-\mathrm{H})-20 \%, \mathrm{C} 6(-\mathrm{H})-30 \%$, O3' $(-\mathrm{H})-25 \%$, unidentified C-centered components < $10 \%$ [19]. Assuming a balance between oxidation and reduction products at low temperature, and taking also a finite concentration of IMTE centers into account, that is difficult to quantify, one may expect the $\mathrm{C} 1(-\mathrm{H})$ radical to be an oxidation product.

\subsubsection{Intermediate temperature stage (irradiation at $80 \mathrm{~K}$ )}

Annealing from 6-10 K up to RT, the EPR spectra of X-ray irradiated sucrose undergo many complex transformations (see Fig. 6.7), which all bear information on the radical chemistry. Transformations between 6 and $50 \mathrm{~K}$ have been discussed in Section 6.4.2. The only notable event in the $50-80 \mathrm{~K}$ range is the disappearance of the O3'(-H) alkoxy radical: all radicals stable at $\mathrm{T}>80 \mathrm{~K}$ are $\mathrm{C}$-centered. This section is confined to the species stable at $80 \mathrm{~K}$ ( $\sim$ liquid $\mathrm{N}_{2}$ temperature), which have been studied quite thoroughly [105]. The annealing (and irradiation) stages between $80 \mathrm{~K}$ and RT are still largely unexplored.

Figure 6.11a shows the EPR spectrum of sucrose after X-ray irradiation at $80 \mathrm{~K}$ and recorded at $80 \mathrm{~K}$ for a magnetic field orientation in the $\{a c\}$ plane close to $\left\langle c^{*}\right\rangle$. It contains contributions of at least 5 distinct radical species, as is clear from the EIE decomposition. The two most prominent components were already produced by irradiation at $10 \mathrm{~K}: \mathrm{C} 5(-\mathrm{H})$, which is stable up to $\sim 100 \mathrm{~K}$, and $\mathrm{C} 1(-\mathrm{H})$, that remains prominently present in the spectra up to $\sim 200 \mathrm{~K}$ (structures, see Fig. 6.8). These radicals very probably correspond with these reported in an earlier study by Ueda et al. [113]. Three additional radical species have not been detected after $10 \mathrm{~K}$ irradiation and are labeled N1, N2 and N3 in Fig. 6.11a.

The ENDOR angular dependence in the $\{a c\}$ plane for these "new" radicals is shown in Fig. 6.11b. Most of these ENDOR transitions could not be followed in other rotation planes. Only for the largest HF coupling of N1 $\left(\mathrm{N} 1-\mathrm{HF}^{+}\right.$and N1$\mathrm{HF}^{-},+$and - refer to the two $M_{S}$ multiplets in which ENDOR transitions were 
observed) and $\mathrm{N} 2\left(\mathrm{~N} 2-\mathrm{HF}^{+}\right.$and $\left.\mathrm{N} 2-\mathrm{HF}^{-}\right) A$ tensors could be reliably determined and the Schonland ambiguity was resolved by measurements a in fourth rotation plane. These tensors are listed in Table 6.7. Both interactions are $\beta$-type. The anisotropy of the second HF interaction of N2 (N2-HF2 $\left.{ }^{+}\right)$and of the HF coupling observed for $\mathrm{N} 3\left(\mathrm{~N} 3-\mathrm{HF}^{+}\right)$suggests that these interactions are $\alpha$-type (or, less likely, $\beta$-hydroxyl type).
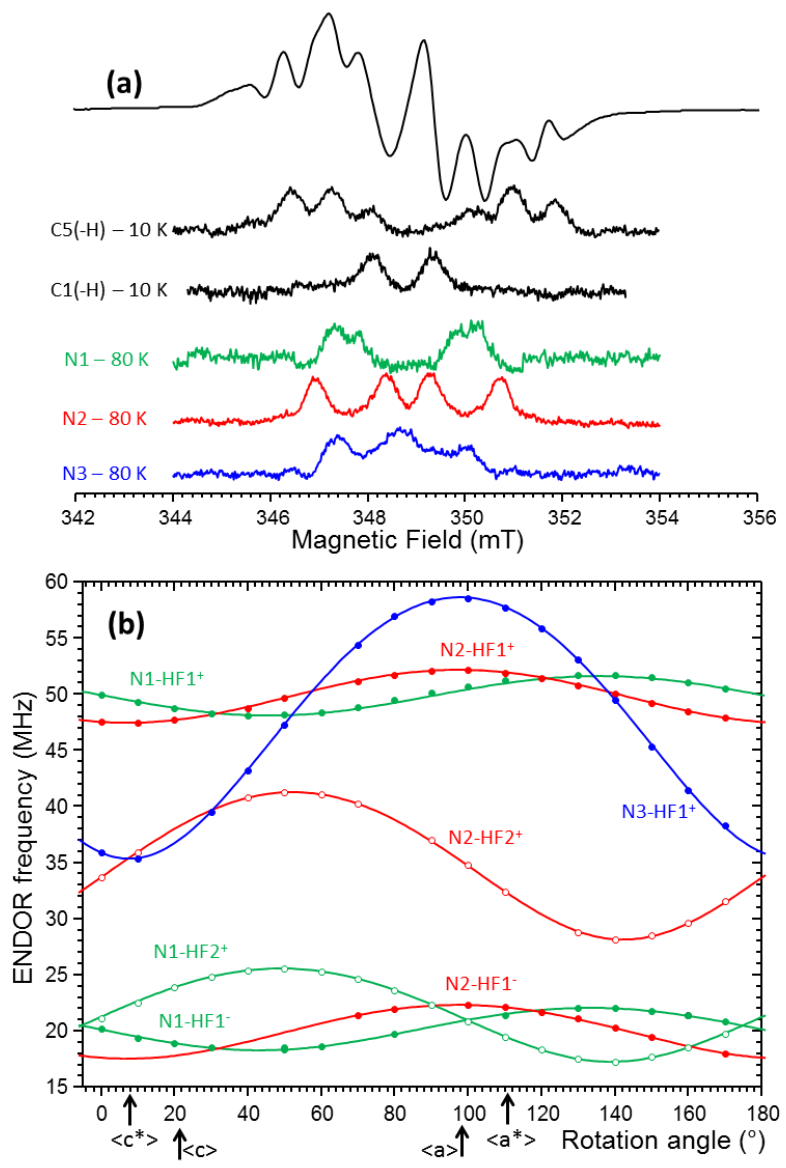

Fig. 6.11. (a) EPR spectrum and its EIE decomposition, recorded at $80 \mathrm{~K}$, of a sucrose single crystal irradiated at $80 \mathrm{~K}$. The magnetic field is oriented in the $\{a c\}$ plane, $8^{\circ}$ from $\left\langle c^{*}\right\rangle$ and $21^{\circ}$ from $\langle c\rangle\left(0^{\circ}\right.$-point of the ENDOR angular dependence). $v_{\mathrm{MW}}=9.77 \mathrm{GHz}$. (b) Angular variation of the ENDOR transitions assigned to species not detected by irradiation at 10K. Filled (largest HF interaction, observed in two $M_{S}$ multiplets for N1 and N2) and open circles (smaller interaction for $\mathrm{N} 1$ and $\mathrm{N} 2)$ represent experimental transitions frequencies, full lines are simulations assuming an angular dependence of transition frequencies of the form $A=\left(\left(A_{\max }\right)^{2} \cos ^{2}\left(\theta-\theta_{0}\right)+\right.$ $\left.\left(A_{\min }\right)^{2} \sin ^{2}\left(\theta-\theta_{0}\right)\right)^{1 / 2} \pm g_{H} \mu_{N} B[105]$ 
Table 6.7. Principal $A$ values $(\mathrm{MHz})$ and direction cosines for the HF couplings determined for the $\mathrm{N} 1$ and $\mathrm{N} 2$ radicals in sucrose, produced by X-ray irradiation at $80 \mathrm{~K}$ and not prominently present after $10 \mathrm{~K}$ irradiation, from angular dependent ENDOR measurements in four rotation planes at $80 \mathrm{~K}$ [105].

\begin{tabular}{ccccccc}
\hline$A$ tensor & $A_{i}$ & $A_{\text {iso }}$ & $A_{\text {aniso, }}$ & $\langle a *\rangle$ & $\langle b\rangle$ & $\langle c\rangle$ \\
\hline \multirow{3}{*}{ N1 - HF1 } & 66.38 & & -5.16 & -0.226 & 0.223 & 0.948 \\
& 69.12 & 71.54 & -2.42 & 0.756 & 0.654 & 0.026 \\
& 79.13 & & 7.59 & -0.615 & 0.723 & -0.316 \\
& 62.95 & & -4.83 & 0.103 & 0.915 & 0.389 \\
N2 - HF1 & 65.74 & \multirow{2}{*}{67.68} & -2.04 & 0.213 & -0.403 & 0.890 \\
& 74.64 & & 6.86 & 0.972 & -0.009 & -0.236 \\
\hline
\end{tabular}

The DFT modeling of these radicals has to date not been exhaustively investigated, but the experimentally determined HF tensors were compared with those calculated (HF tensor calculation on single molecule cut from periodically optimized structures) for all possible $\mathrm{C}$-centered $\mathrm{H}$-abstraction radicals in sucrose.

Table 6.8. Principal $A$ values (MHz) and direction cosines calculated for the radical produced by $\mathrm{H}$-abstraction at the $\mathrm{C} 5$ ' carbon atom of sucrose. Angles between experimental and calculated directions are given in degrees [105].

\begin{tabular}{|c|c|c|c|c|c|c|c|c|}
\hline $\mathrm{C}^{\prime}(-\mathrm{H})$ & $A_{i s o}$ & $A_{\text {aniso, } i}$ & $<a^{*}>$ & $<b>$ & $<_{c}>$ & $\Delta A_{\text {iso }}$ & $\Delta A_{\text {anis }, i}$ & $\delta$ \\
\hline \multirow{3}{*}{$\beta$-H4' } & \multirow{3}{*}{64.55} & -5.11 & -0.237 & 0.198 & 0.951 & & 0.05 & 1.6 \\
\hline & & -2.67 & 0.725 & 0.687 & 0.038 & -6.99 & -0.25 & 2.6 \\
\hline & & 7.78 & -0.646 & 0.699 & -0.307 & & 0.19 & 2.3 \\
\hline \multirow{3}{*}{$\beta$-H6'a } & \multirow{3}{*}{9.40} & -6.12 & 0.103 & 0.915 & 0.389 & & & \\
\hline & & -3.65 & 0.213 & -0.403 & 0.890 & & & \\
\hline & & 9.77 & 0.972 & -0.009 & -0.236 & & & \\
\hline \multirow{3}{*}{$\beta$-H6'b } & \multirow{3}{*}{7.04} & -6.50 & 0.836 & -0.345 & 0.427 & & & \\
\hline & & -3.59 & 0.348 & 0.935 & 0.073 & & & \\
\hline & & 10.09 & -0.424 & 0.088 & 0.901 & & & \\
\hline
\end{tabular}

The $\beta$-interaction of $\mathrm{N} 1$ is found to correspond very well with the largest interaction calculated for the $\mathrm{C}^{\prime}{ }^{\prime}(-\mathrm{H})$ model, as can be seen in Table 6.8. Two more appreciable HF interactions are predicted for this radical. One of these, (probably $\beta$-H6'a) can account for the second coupling observed in the ENDOR angular dependence in the $\{a c\}$ plane $\left(\mathrm{N} 1-\mathrm{HF}^{+}, \mathrm{Fig} .6 .11 \mathrm{~b}\right)$ and for the small splitting in the EIE spectrum (Fig. 6.11a). Although further experimental and computational confirmation is desirable, the proposed model for $\mathrm{N} 1$ is already convincing. It should also be noted that even after irradiation at $10 \mathrm{~K}$, very faint transitions of the largest $\mathrm{HF}$ interaction of $\mathrm{N} 1$ have been detected in the X-band ENDOR spectra. 
For N2, none of the $\mathrm{H}$-abstraction models yield reasonable agreement with the characterized $\beta$-coupling. Very probably, the model for this radical is more complex than a simple $\mathrm{H}$-abstraction. Insufficient data are available for N3 to allow even a guess of its radical model.

\subsubsection{Immediately after RT irradiation}

In Fig. 6.7, one can see that the spectrum of sucrose irradiated at low temperature, after anneal to RT differs strongly from that of the final stable radiation products. The latter spectra are only obtained after keeping the irradiated crystal for several hours at RT. An initial characterization of the metastable stage immediately after RT irradiation [51], in which EPR and FF-ENDOR were combined with statistical EPR spectrum decomposition based on Maximum Likelihood Common Factor Analysis (MLCFA [6, 114]), revealed that

i) the final stable radicals (see Section 6.4.5) are already present immediately after irradiation at RT or $273 \mathrm{~K}$.

ii) the spectra immediately after irradiation are dominated, however, by two other radical components, labeled $\mathrm{U} 1$ and $\mathrm{U} 2$ in Ref. [51].

iii) the U1 and $\mathrm{U} 2$ radicals decay at RT, with characteristic double exponential decays having time constants $\tau_{1}=850 \mathrm{~s}$ and $\tau_{2}=4700 \mathrm{~s}$, and apparently without production of stable radical components. This conclusion was obtained by MLCFA decomposition of the Q-band EPR spectra recorded while monitoring the spectral transformation during isothermal annealing at RT. This, at least in part, explains why, for reliable dose estimations, irradiated sucrose EPR spectra can only be recorded after a sufficiently long waiting time (typically 2-3 days) [30].

iv) the stable radical concentration is about half the total concentration of paramagnetic centers measured immediately (within $5 \mathrm{~min}$.) after irradiation at RT or $273 \mathrm{~K}$.

A thorough characterization of the $\mathrm{U} 1$ and $\mathrm{U} 2$ radicals combining ENDOR and EIE with DFT calculations is presently ongoing [56]. The EPR and EIE spectra for the two radicals in three perpendicular magnetic field orientations are shown in Fig. 6.12. U1 exhibits three resolved HF interactions: one $\alpha$-type interaction $\left(\mathrm{A}_{\text {iso }}\right.$ $\sim 1.8 \mathrm{mT})$, one $\beta$-type of intermediate magnitude $\left(\mathrm{A}_{\text {iso }} \sim 1.5 \mathrm{mT}\right)$ and a smaller coupling that is only resolved in the EPR spectra at magnetic field orientations near the $\langle a\rangle$ and $\left\langle a^{*}\right\rangle$ axes $(0.5-0.6 \mathrm{mT})$. The U2 radical exhibits two strong $\beta$ type $\mathrm{HF}$ interactions $\left(\mathrm{A}_{\text {iso, } 1} \sim 3.1 \mathrm{mT}\right.$ and $\left.\mathrm{A}_{\text {iso, } 2} \sim 2.9 \mathrm{mT}\right)$. In the EIE spectrum with $\mathrm{B} / /\left\langle_{c}>\right.$ a barely resolved additional splitting of $\sim 0.4 \mathrm{mT}$ is observed. It is noteworthy that for all measured U1 and U2 EIE spectra $g$ factors in the 2.002-2.003 range are found (see simulation parameters in the caption). This result suggests 
that the $g$ tensors for these radicals are fairly isotropic, and that these EPR components most probably are due to (hydroxy)alkyl radicals, without significant spin delocalization onto oxygen (see Section 6.3.2). Preliminary comparisons between DFT calculated and experimental HF tensors show that neither of these radicals correspond to simple $\mathrm{H}$-abstraction radicals on $\mathrm{C}$-atoms.

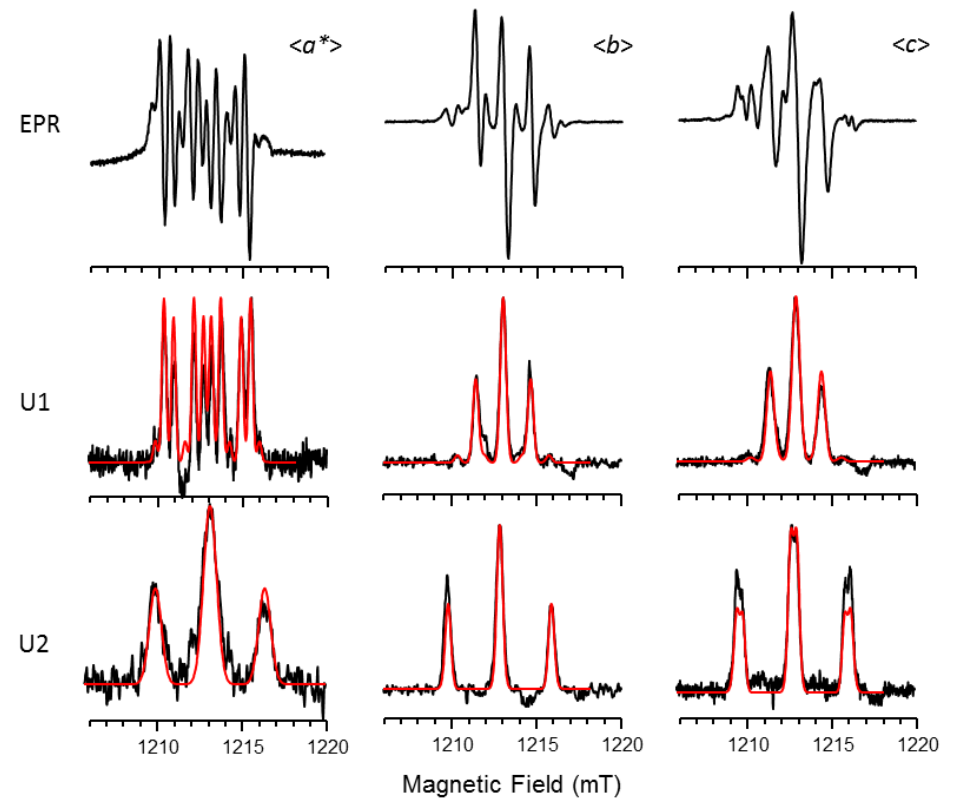

Fig. 6.12. Q-band EPR and EIE spectra ( $50 \mathrm{~K}$, black - experimental and red - simulation) of sucrose, short time after irradiation at ice-water temperature. $v_{\mathrm{MW}}=34.00 \mathrm{GHz}[56,106]$.

SH parameters for U1 $-\left\langle a *>: g=2.0027, A_{1}=2.72 \mathrm{mT}, A_{2}=1.66 \mathrm{mT}, A_{3}=0.57 \mathrm{mT} ;<b>\right.$ : $g=2.0026, A_{1}=1.59 \mathrm{mT}, A_{2}=1.55 \mathrm{mT} ;<c>: g=2.0027, A_{1}=1.46 \mathrm{mT}, A_{2}=1.66 \mathrm{mT}$.

SH parameters for U2 - $\left\langle a^{*}>: g=2.0022, A_{1}=3.36 \mathrm{mT}, A_{2}=3.03 \mathrm{mT} ;\langle b\rangle: g=2.0029\right.$, $A_{1}=3.04 \mathrm{mT}, A_{2}=2.99 \mathrm{mT} ;<_{c}>: g=2.0029, A_{1}=3.14 \mathrm{mT}, A_{2}=2.89 \mathrm{mT}, A_{3}=0.40 \mathrm{mT}$.

\subsubsection{Final stable stage}

The X-band RT EPR spectrum of irradiated sucrose powder, long time after irradiation (typically 3 days), is shown in Fig. 6.13. This spectrum looks deceivingly simple: it appears to be dominated by a component exhibiting a doublet HF splitting with a weaker triplet structure on both components, very likely due to two additional smaller HF interactions. The asymmetry between the low and high-field HF lines and the occurrence of side-lines (indicated with arrows), however, suggest that the radical composition may be more complicated. This is confirmed 
when recording the spectrum at higher microwave frequencies, as shown in Fig. 6.13 at Q-band.
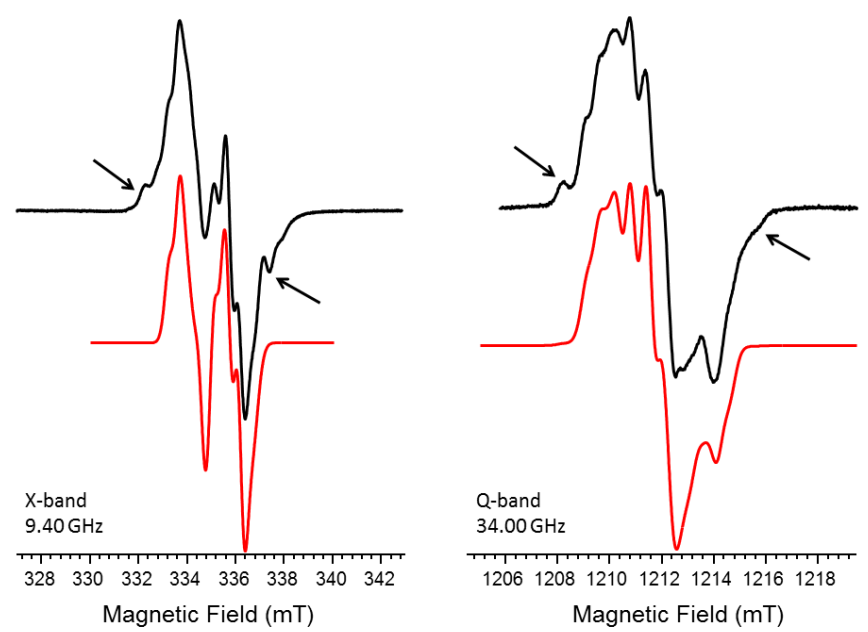

Fig. 6.13. Stable RT X- and Q-band powder EPR spectrum of irradiated sucrose ( $40 \mathrm{kGy})$. Black - experiment ; red - simulations, assuming a radical composition T1:T2:T3 of 1:0.72:0.48. $v_{\mathrm{MW}}=34.00 \mathrm{GHz}$

Using ENDOR at RT, Sagstuen et al. were able to characterize two distinct radical types [5]. MLCFA decomposition of RT X-band powder EPR spectra in a dose series, on the other hand, revealed three dominant contributions with distinct dose responses [6]. ENDOR studies at low temperature (55 K [6] and $110 \mathrm{~K}$ [55]) of crystals irradiated at RT allowed to distinguish, indeed, three types of radicals, labeled T1-T3, all exhibiting one large and two small proton HF couplings. Comparison between experimental and calculated HF tensors for these couplings allowed to identify the two radical models, depicted in Fig. 6.14.
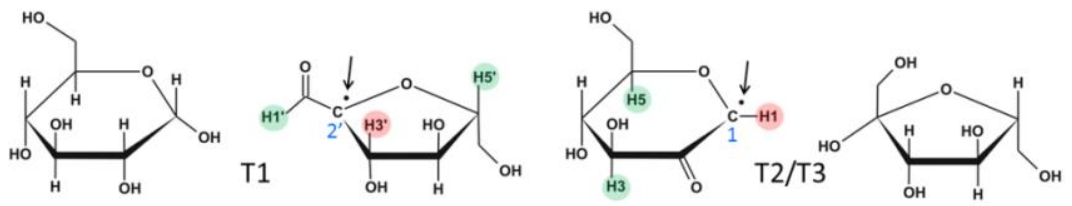

Fig. 6.14. Models for the T1 (left) and T2/T3 (right) stable radicals in sucrose [8, 9]. The central carbon on which the unpaired electron is mainly localized, is numbered and indicated with an arrow. The proton exhibiting the strongest HF interaction with the unpaired electron is indicated with a red circle, the protons yielding the two resolved $\gamma$-couplings with green circles.

The model for T1 was already shown in Fig. $6.6\left(\mathrm{M}\left(\mathrm{C} 2^{\prime}\right) \mathrm{c}\right)$. It exhibits one $\beta$ type and two $\gamma$-type HF couplings. T2 and T3 have very similar HF couplings (one $\alpha$ and two $\gamma$-types) and are two slightly different conformations of the same $\mathrm{C} 1$ - 
centered radical, formed by rupture of the glucose-fructose glycosidic bond [8]. The DFT calculations for the common T2/T3-model in Fig. 6.14 agree significantly better with the results for T2, suggesting that $\mathrm{T} 3$ has basically the same structure but with a perturbation in its environment.

It is interesting to note that these two models, and hence also the three stable radical types in sucrose, have very similar characteristics, almost like mirror images in the fructose (T1) and glucose (T2/T3) units. They are both formed by glycosidic bond cleavage and have their unpaired electron mainly localized on a $\mathrm{C}$ atom next to the ring oxygen, with carbonyl formation in a $\beta$-position. These features are indicative of a formation process involving $\beta$-elimination (see Section 6.6). The resulting strong delocalization of the unpaired electron is evidenced by the atypically small HF couplings with $\alpha$ - or $\beta$-protons, and by the atypically large interaction with distant $\gamma$-protons. In addition, the $g$ tensors (experiment and calculations) that were recently reported for these radicals [57] reveal an enhanced anisotropy, with principal $g$ shifts from the free-electron value up to 0.0045 .

In order to understand the difference between the low temperature $(50 \mathrm{~K}[6]$, $110 \mathrm{~K}$ [55]) and RT ENDOR results, the temperature dependence of the ENDOR spectra at certain magnetic field orientations was recorded [55]. This indicated that all determined HF tensors are only slightly temperature-dependent and that near RT the difference between the T2 and T3 radicals appears to vanish. As a consequence, the three radical types detected with ENDOR at low temperature do not necessarily correspond to the three components deduced from the MLCFA decomposition of the RT powder EPR spectra of a dose series. Moreover, if certain radical types exhibit the same dose response, MLCFA would not be able to distinguish between them. Spectrum simulations using the $110 \mathrm{~K}$ ENDOR data for the T1-T3 (also shown in Fig. 6.13) reproduce the central parts of the RT powder EPR spectra fairly well, but fail in reproducing the wing-lines (indicated by arrows in the spectra), even though transitions often regarded as forbidden were included in the simulations [57, 58]. This was also noticed by Georgieva et al. [7] in the high-frequency powder EPR spectra. For most magnetic field orientations such wing-lines are also seen in the X and Q-band single crystal EPR spectra. Hence, certain (essential) contributions to the spectra to date still remain unidentified.

The metastable U1 and U2 components (see Section 6.4.4) might in principle be considered as responsible for these wing-lines. FF-ENDOR and statistical decomposition studies, however, suggest that these components decay completely at RT [51], whereas the missing spectral component has a rather low, but stable intensity. On the other hand, the single crystal ENDOR spectra of irradiated sucrose in the stable stage reveal the presence of at least one additional radical component exhibiting 3 or 4 resolved proton HF interactions. This is clearly seen in the FFENDOR spectra in Fig. 6.2. EPR and EIE spectra for this component are shown in Fig. 6.15 [56]. These indicate that the corresponding radical exhibits one large $\beta$ type HF interaction and several smaller couplings, one of which probably has in- 
termediate magnitude $(\sim 30-40 \mathrm{MHz})$. The EIE spectra also show that, similar to T1-T3, this radical has an appreciable $g$ anisotropy, indicating spin delocalization onto ring and/or carbonyl oxygen. A complete characterization of this radical is presently ongoing [56]. Comparison of the powder EPR (and possibly also ENDOR) spectra with spectrum simulations will allow to verify if this radical actually corresponds to the presently unknown components in the powder spectra.
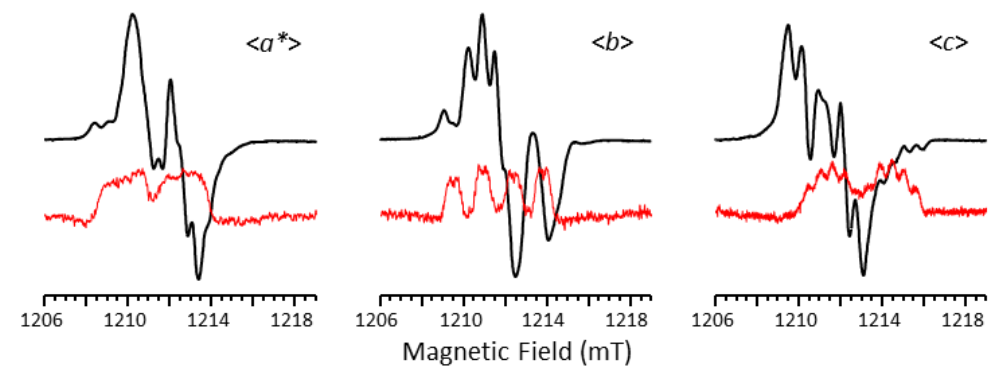

Fig. 6.15. EPR (black) and EIE spectra (red, recorded on the ENDOR line above $90 \mathrm{MHz}$ at $1210 \mathrm{mT}$ static field) of RT irradiated sucrose (long time after irradiation) for magnetic field orientations along $\left\langle a *>,\left\langle b>\right.\right.$ and $\langle c\rangle$; T $=110 \mathrm{~K}, v_{M W}=34.00 \mathrm{GHz}$.

\subsubsection{Pulse annealing results - pitfalls in the analysis}

In addition to the shape (radical composition), the intensity of the EPR spectra provides relevant information on the radiation chemistry of carbohydrates. In the introduction to this section, it is explained that for reliable comparison of intensities, EPR spectra have to be recorded at the same temperature. Pulse annealing experiments (although very time consuming) are most appropriate for this. For sucrose a pulse annealing experiment covering the complete $\sim 6 \mathrm{~K}$ to RT temperature range has not been performed yet. The results of pulse annealing experiments up to RT after $80 \mathrm{~K}$ irradiation [105] are summarized in Fig. 6.16. The total double intensity data points are averages over pulse annealing experiments performed for four different magnetic field orientations in the $\{a c\}$ plane at X-band. In order to get a more complete picture of the evolution of the radical concentration, results from the previous sections should also be taken into account.

In Section 6.4.2 it was shown that in the 6-50 K range the total EPR intensity remains essentially constant and in the $50-80 \mathrm{~K}$ range the $\mathrm{O} 3$ ' $(-\mathrm{H})$ alkoxy radical is annealed out. Finally, when keeping the crystal for a prolonged time at RT a further decrease in the total intensity by about $50 \%$ is expected (Section 6.4.4). One may conclude that only about $10 \%$ of the original radical concentration created at $6 \mathrm{~K}$ "survives" to the RT stable stage. This implies that identifying radicals with low concentrations at low temperatures may be important, as they may be involved in the formation mechanisms of the stable radicals. On the other hand, cer- 
tain (majority) radical components at low temperature may not be precursor to any of the radicals stable at higher temperatures. In addition, for a complete understanding of the radiation chemistry, recombination processes and diamagnetic radiation products should also be properly identified.

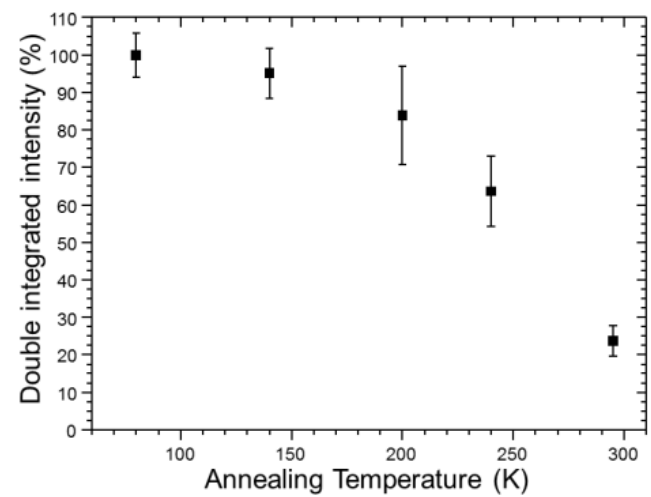

Fig. 6.16. Double integrated total intensity of the EPR spectrum of sucrose irradiated at $80 \mathrm{~K}$, measured at $80 \mathrm{~K}(9.78 \mathrm{GHz})$ as a function of annealing temperature in pulse annealing experiments [105]. In each step the sample was heated to the annealing temperature, kept there for $\sim 10$ min. after stabilization, and quenched to $80 \mathrm{~K}$. EPR spectra were then recorded after a stabilization time of $\sim 10 \mathrm{~min}$. Filled squares indicate averages of data points collected for magnetic field alignments along $\langle a\rangle,\langle a *\rangle,\langle c\rangle$ and $\left\langle c^{*}\right\rangle$. Error bars indicate 2 times the experimental standard deviation.

The discussion in Section 6.4 clearly demonstrates that, in spite of the very detailed investigation of the paramagnetic radiation products, even for sucrose several features are still not properly understood. Hence, the detailed reaction sequences leading to the stable radicals cannot be elucidated yet. Nevertheless, the results of our investigations allow proposing certain very likely reaction schemes for some of the stable radicals, as will be discussed in Section 6.6.

Table 6.9 summarizes the results of the radical identification studies on sucrose, listing for each irradiation stage the species characterized by EMR, along with models, wherever these are available, and references to the appropriate literature. In the models, the protons are labeled whose HF interactions have been characterized in the EMR studies. In Section 6.5 similar tables are presented for the other carbohydrates taken up in our studies (see Table 6.1 for atom labeling). For each carbohydrate, a discussion of the results is also given. 
Table 6.9. Radiation-induced radicals in sucrose

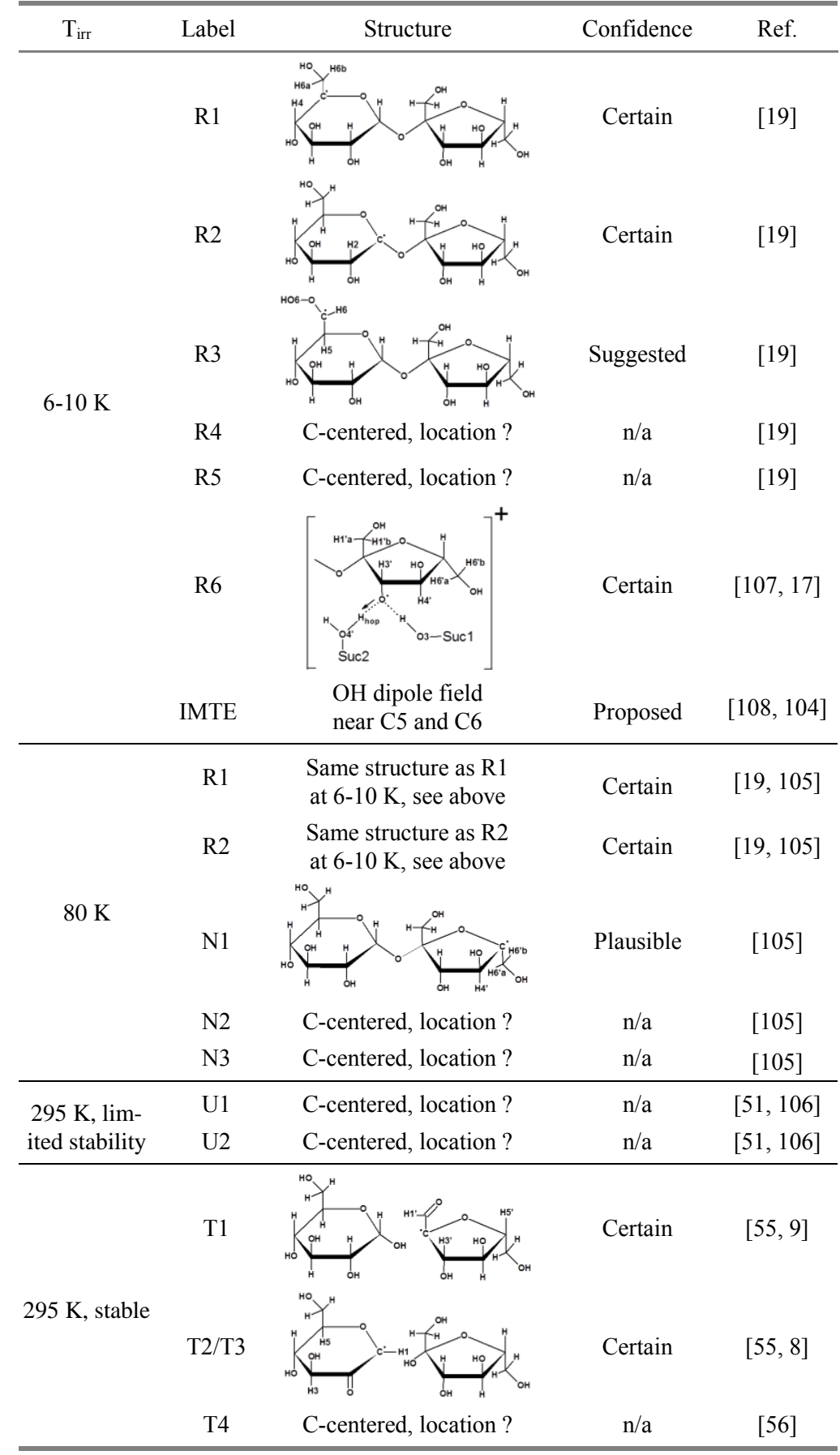




\subsection{Results for other carbohydrates}

\subsubsection{Dipotassium glucose-1-phosphate}

Table 6.10. Radiation-induced radicals in K2G1P

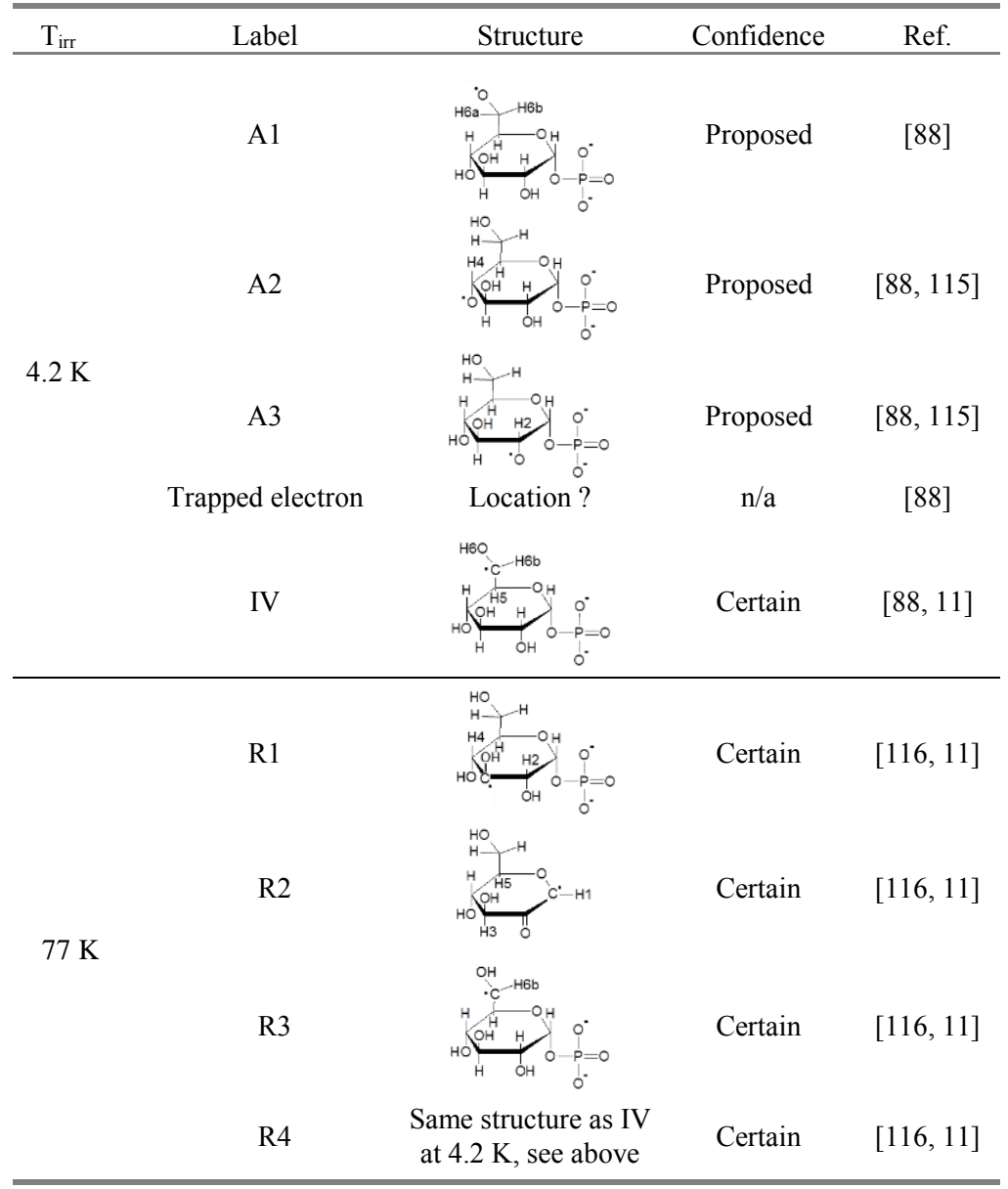

Glucose-1-phosphate is a particularly interesting model system for studying radiation damage to DNA, because it also contains sugar-phosphate bonds and strand breaks in DNA involve rupture of this bond. The primary radiation-induced radicals in K2G1P after exposure at $4 \mathrm{~K}$ were studied by Locher and Box [88], who reported 3 different alkoxy radicals $(\mathrm{A} 1-\mathrm{A} 3, \mathrm{~A} 1=\mathrm{O} 6(-\mathrm{H}))$, an IMTE and a C6(-H) hydroxyalkyl radical (IV) [88]. The assignment of the latter was explicitly 
confirmed by DFT calculations [11]. A meta-analysis of the data, including new crystal structure determinations [117], and considering the Schonland ambiguity $[115,118]$, showed that the assignment of A1 is reasonable and that A2 and A3 most probably correspond to alkoxy radicals at $\mathrm{O} 4$ and $\mathrm{O} 2$, respectively. In partially deuterated crystals, only A3 was observed together with the IMTE and $\mathrm{C} 6(-\mathrm{H})$ [88]. Several other radicals were formed at LT, but not further analyzed. Also, no thermal annealing or light bleaching studies were reported. Interestingly, no $\mathrm{P}$-centered radicals were produced by irradiation at this temperature.

Bungum et al. [118] and Sanderud and Sagstuen [119] studied the structures of P-centered radicals in this compound. They found that such radicals are not produced by irradiation at $77 \mathrm{~K}$, but do appear after subsequent anneal to RT or when irradiating crystals at (close to) RT. Hence, sugar radicals very probably act as precursors for the P-centered radicals. This was one of the motivations for the elaborate study of C-centered radicals after X-ray irradiation at $77 \mathrm{~K}$ by De Cooman et al. [116] and Pauwels et al. [11]. Four C-centered radicals were identified in these studies. Other $\mathrm{C}$-centered radicals were detected in the EPR spectra but could, for technical reasons, not be studied with ENDOR. The R2 radical, with the most prominent contribution to the EPR spectrum, has a structure very similar to that of T2/T3 in sucrose. It is also interesting to note that a C5-centered radical, that had been proposed as precursor for the phosphoryl radical detected at RT in K2G1P [119], was not identified in these studies.

\subsubsection{Trehalose dihydrate}

To evaluate to which extent the findings of the sucrose studies can be generalized, we have undertaken an analogous study on the structurally similar trehalose (diglucose). The first EPR spectra from RT irradiated trehalose crystals had already been investigated at $77 \mathrm{~K}$ by Gräslund and Löfroth in the 1970's [4]. A 1:2:1 triplet with a $3 \mathrm{mT}$ HF splitting was assigned to a $\mathrm{C} 3$ or $\mathrm{C} 3$ '-centered radical.

Samskog and Kispert extended this EPR work to radicals formed after irradiation at $3 \mathrm{~K}$ [111] and $77 \mathrm{~K}$ [120]. Irradiation at $3 \mathrm{~K}$ led to three (close to) primary radicals, proposed to be an IMTE (radical I), an O4' alkoxy radical (radical III) and again a 1:2:1 triplet (radical II), also found after irradiation at $77 \mathrm{~K}$ and assumed to be the same as found previously [4]. After irradiation at $10 \mathrm{~K}$ (Tarpan et al. [20]), the trapped electron could no longer be detected at variance with the radicals II and III, relabeled as R1 and R4 respectively. A thorough ENDOR and DFT analysis convincingly modified the identification of the $1: 2: 1$ triplet II/R1 to a $\mathrm{C} 5$ '-centered radical, whereas the III/R4 assignment to the O4' alkoxy radical appeared plausible but still not conclusive. Two more radicals, R2 and R3, were convincingly identified as $\mathrm{C} 5$ and $\mathrm{O} 2$-centered radicals, respectively [20]. 
Table 6.11. Radiation-induced radicals in trehalose dihydrate

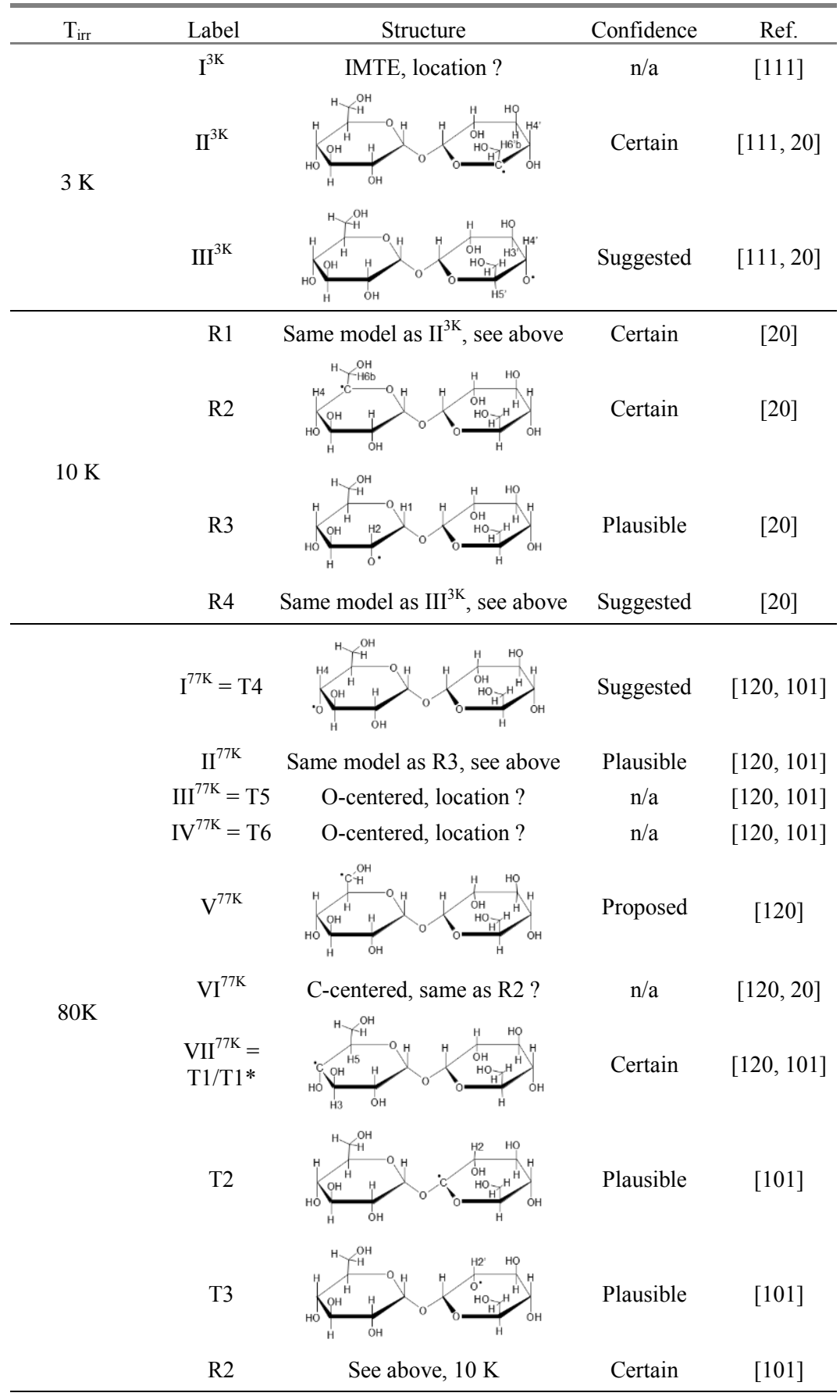




RT, long term $\begin{array}{lll}\text { RT, limited } \\ \text { stability }\end{array}$

Tarpan et al. [101] also extended the EPR investigations of Samskog and Kispert after irradiation at 77K [120] with ENDOR and DFT. Not surprisingly, the strongly composite EPR spectrum was for a major part interpreted in terms of the same (at least) 7 components, albeit again with a different labeling. Apart from the $\mathrm{O} 2$ alkoxy radical mentioned above, all assignments by Samskog and Kispert have been questioned and/or corrected by the combined ENDOR/DFT investigations [101]. Again a 1:2:1 triplet was prominently present and convincingly assigned to two slightly different conformations of a $\mathrm{C} 4(-\mathrm{H})$ hydrogen-abstracted radical (VII or $\mathrm{T} 1 / \mathrm{T} 1 *)$. In spite of its similar appearance, the triplet has another origin than the one mentioned in the lower temperature studies (both different from the radical after RT-irradiation, see also [4]). In addition, mainly based on their characteristic $g$ tensors, the formation of 4 different alkoxy type radicals is quite certain, although their location remains mostly unknown or speculative (III, IV or T5, T6). Radicals T3 and T4 (or I) have been tentatively identified as O2' and O4-centered respectively. The stronger $g$ anisotropy of T5,T6 as compared with T3 and T4 could not be explained by calculations yet. Finally, the T2 radical was only reported by Tarpan et al. [101] and is probably due to a $\mathrm{Cl}(-\mathrm{H})$ radical.

In another combined ENDOR/DFT study, De Cooman et al. [12] provided firm proof for two H-abstracted radicals with limited stability, created by RTirradiation. The I1 and $\mathrm{I} 2$ spectra have been assigned to $\mathrm{C} 3$ ' and $\mathrm{C} 2$-centered radicals respectively, the latter with additional formation of a carbonyl group at C3. Similar H-abstracted radicals occur in irradiated glucose [16]. The stable radicals S1 and S2 could only be tentatively identified. Like for I2, C2 seems to be the most likely site for the radical center of S1 and S2. 


\subsubsection{Fructose}

It could a priori be expected that monosaccharides as, e.g., fructose, glucose and sorbose, would lead to EMR spectra that are simpler and easier to interpret than the disaccharides sucrose and trehalose. Therefore, about one decade ago, our group started an extensive EMR investigation on $\beta$-D-fructopyranose. This was also the first sugar for which a combined ENDOR/DFT approach was applied. It appeared that, contrary to expectations, a realm of radicals are detected in fructose and as a consequence, several identification problems are still unsolved.

Table 6.12. Radiation-induced radicals in $\beta$-D-fructose

\begin{tabular}{|c|c|c|c|c|}
\hline $\mathrm{T}_{\text {irr }}$ & Label & Structure & Confidence & Ref. \\
\hline \multirow{2}{*}{$10 \mathrm{~K}$} & $\mathrm{~T} 1 / \mathrm{T} 1 *$ & & Certain & {$[18]$} \\
\hline & $\mathrm{T} 2$ & & Plausible & {$[18]$} \\
\hline \multirow{2}{*}{$80 \mathrm{~K}$} & $\mathrm{R} 1 / \mathrm{R} 1 *$ & $\begin{array}{c}\text { Same model as } \mathrm{T} 1 / \mathrm{T} 1 * \text { at } \\
10 \mathrm{~K} \text {, see above }\end{array}$ & Certain & [91] \\
\hline & $\mathrm{R} 2 / \mathrm{R} 3$ & & Certain & {$[91]$} \\
\hline $160 \mathrm{~K}$ & Q1 & & Plausible & {$[81]$} \\
\hline \multirow{4}{*}{ RT, unstable } & $\operatorname{Im} 1$ & & Plausible & {$[81]$} \\
\hline & $\operatorname{Im} 3$ & C5-centered, broken ring ? & Proposed & {$[81]$} \\
\hline & $\operatorname{Im} 4$ & & Plausible & {$[81]$} \\
\hline & $\operatorname{Im} 5$ & $\mathrm{C} 2$-centered, broken ring? & Proposed & {$[81]$} \\
\hline
\end{tabular}




R6 stable

Fructose and sorbose are structurally very similar and Ueda [121] was the first to systematically compare the EPR spectra of these materials both on powders and single crystals, after irradiation at $77 \mathrm{~K}$ and at RT. These authors found that irradiating fructose and sorbose at $77 \mathrm{~K}$ indeed leads to similar spectra whereas RT irradiation gives rise to significantly different spectra. Heating sorbose to $100^{\circ} \mathrm{C}$ for $10 \mathrm{~min}$. however, makes the spectra of both sugars similar again. Without having access to ENDOR or DFT methods and undoubtedly underestimating the complexity of the spectra, these authors proposed a C5-centered model for one of the radicals formed after $77 \mathrm{~K}$ irradiation [121]. In the remainder of this section, only fructose radicals will be discussed.

In their first study on fructose, Vanhaelewyn et al. [122] confined themselves to stable radicals in RT irradiated powders and single crystals. From a statistical MLCFA study on EPR powder spectra, it became clear that at least four stable radicals contributed to the spectra. This finding was confirmed later by ENDOR and EIE investigations [14, 81]. Two dominant radicals, labeled F1/F2, were found to exhibit quite comparable $\mathrm{HF}$ interactions and, based on single molecule DFT calculations, a C2-centered model was proposed for both of them [89, 122] (an alternative labeling of the fructose atoms was used in that work). Although Tarpan et al. in later work also determined the $g$ tensors of F1/F2 and applied selective ${ }^{13} \mathrm{C}$ enrichment in fructose powders $[13,14,81]$, the identity of these main stable radicals remains uncertain. Using the most advanced DFT methods presently available, a $\mathrm{C} 3(-\mathrm{H})$ model, featuring a neighboring carbonyl group and possibly also ring opening, seems the most plausible. Still, overall good agreement between experimental and computational data has not been reached [14, 81].Also radicals F3 and F6 were proposed to be $\mathrm{C} 3$-centered. F6 is $\mathrm{H}$-abstracted with a carbonyl group at $\mathrm{C} 2$, whereas a $\mathrm{C} 3(-\mathrm{OH})$ model seems plausible for radical $\mathrm{F} 3[14,81]$. Another pair $\mathrm{F} 4 / \mathrm{F} 5$ has been assigned to $\mathrm{C} 2(-\mathrm{OH})$ radicals in slightly different conformations, accompanied by carbonyl formation at $\mathrm{C} 3$. The radicals $\mathrm{F} 1 / \mathrm{F} 2$, F4/F5 were demonstrated to be present immediately after RT-irradiation (Im2, 
Im6) $[14,81]$. Plausible models were found by Tarpan et al. for two unstable radicals: $\operatorname{Im} 1$ and $\operatorname{Im} 4$ have been assigned to $\mathrm{C} 4(-\mathrm{H})$ and $\mathrm{C} 2(-\mathrm{OH})$, respectively. The identifications of $\operatorname{Im} 3$ and $\operatorname{Im} 5$ remain far more speculative (broken-ring radicals centered at C5 and C2) [81].

In search of (close to) primary radicals, Tarpan et al. [18] and Vanhaelewyn et al. [91] also investigated fructose single crystals after X-irradiation at $10 \mathrm{~K}, 80 \mathrm{~K}$ and annealing to $160 \mathrm{~K}$. All proposed models for these radicals can be considered to be highly reliable if not certain. Both after irradiation at $10 \mathrm{~K}$ and $80 \mathrm{~K}$ a prominent pair (labeled $\mathrm{T} 1 / \mathrm{T} 1 *$ and $\mathrm{R} 1 / \mathrm{R} 1 *$ respectively) has been identified (again) as the $\mathrm{C} 3(-\mathrm{H})$ species. After $10 \mathrm{~K}$ irradiation, a second simple $\mathrm{H}$-abstracted radical, $\mathrm{C} 5(-\mathrm{H})$ was reported (T2). Another $\mathrm{C} 3$-centered radical (two conformations) that was discovered after irradiation at $80 \mathrm{~K}(\mathrm{R} 2 / \mathrm{R} 3)$, remained stable up to at least $160 \mathrm{~K}(\mathrm{Q} 2)$. Ring opening and formation of a carbonyl group appeared essential to reach excellent agreement between experimental and computational data. It is clear that the convincing identification of these radicals puts severe constraints on the precise identity of the F1/F2 C3-centered radicals (also assumed to have a broken ring) mentioned above. The $\mathrm{C} 3$-centered F3 and F6 radicals were proposed to have an intact ring $[81,91]$. Finally the last fructose radical with a probable identification $(\mathrm{Q} 1)$, stable up to at least $160 \mathrm{~K}$, is a $\mathrm{C} 1(-\mathrm{H})$ radical species [81].

\subsubsection{Sorbose}

Table 6.13. Radiation-induced radicals in $\alpha$-L-Sorbose

\begin{tabular}{lllll}
\hline $\mathrm{T}_{\text {irr }}$ & Label & Confidence & Ref. \\
\hline 295 & $\mathrm{R} 1 / \mathrm{R} 1$ 1 & Plausible & {$[10]$}
\end{tabular}

The chemical structure of sorbose is nearly identical to that of fructose and a priori many similarities in radical formation could be expected, as were also discovered by Ueda [121] (see Section 6.5.5). However, the EMR study of this material appeared experimentally extremely complex and lack of complete $g$ and HF data for most of the components prohibits detailed comparison with DFT modeling. Extensive MLCFA analyses, exploiting differential radical annealing between RT and $435 \mathrm{~K}$, revealed more than 10 stable radicals after RT irradiation. In spite of considerable efforts hardly any complete HF tensor could be determined even using ENDOR. The models, very tentatively proposed for the stable radicals, usually involve ring-openings and the formation of a carbonyl group adjacent to the radical center $[123,124]$. As a result of these studies, however, excellent simulations of the RT EPR spectra became possible. 
So far, the best characterized and identified radicals are R1/R1', which dominate the EPR spectrum after RT irradiation. They are very probably $\mathrm{C} 3(-\mathrm{H})$ species. The difference between $\mathrm{R} 1$ and $\mathrm{R} 1$ ' has been ascribed to the structural $\alpha / \beta$ disorder in the sorbose lattice, which may in part be responsible for the problems encountered in characterizing the other radical species in the EMR analyses [10].

In view of the very limited information on radical structures, no attempts are made to interpret the radical chemistry of sorbose in Section 6.6.

\subsection{Radical formation mechanisms and radiation chemistry}

In Sections 6.4 and 6.5, the structures of free radicals formed after irradiation at very low temperatures $(6-10 \mathrm{~K}, \mathrm{LT})$, intermediate temperatures (typically $80 \mathrm{~K}$ ) and at RT have been presented. For the interpretation of these results it is important to keep in mind that the radical products formed by irradiation at a given intermediate or high temperature are the same as those obtained after irradiation at $6-10 \mathrm{~K}$ followed by warming to this temperature. This strongly suggests an internal connection between the radicals produced at LT and those observed at a higher temperature, to be described by chemical conversion processes. In Sections 6.1 and 6.4 we mentioned this as a motivation for studying radiation products after irradiation at various temperatures.

The structures depicted in Tables 6.9-13 may be considered as dots in a complex drawing of the entire radiation processes picture, dots which remain to be connected by lines to obtain the full picture. The tools for completing the scheme are presented by radiation chemistry $[125,126]$. Once hypotheses on actual processes connecting two or more structures are formulated, they should be probed by kinetic studies or quantum chemical modeling. However, constructing schemes that connect species stable at different temperatures, solely based on their structure assignments, presents several pitfalls. First, EMR techniques only detect paramagnetic species: diamagnetic products from a given reaction are not observable. Secondly, when only starting and end-point in a reaction chain are known, there may be several possible routes between them. EMR spectroscopy alone will not allow deciding which route is actually followed. Recent results obtained for the amino acid alanine illustrate the important role of quantum chemical modeling in this: the entire sequence of processes in the reduction chain could finally be mapped by using advanced DFT calculations [127] even if the basic radical structures have been known for many years [128]. Another example is the recent advances made using mass spectroscopy and high-performance liquid chromatography to understand the early radiation-induced processes in DNA [129, 130]. Thirdly, depending on the temperature (thermal energy), a given primary product may proceed along different reaction paths, sometimes leading to different end products. Finally, in too 
many instances there are no experimental indications available showing which radical observed at higher temperature is connected to which LT radical species.

The exposure of organic matter to ionizing radiation (photons, electrons) initiates a number of processes in the material. The field of radiation physics describes these processes in great detail [131], far beyond the scope of this chapter. It is sufficient to state that the deposition of radiation energy in organic matter mainly results in ionizations and excitations. An ionization event creates a hole and a secondary electron with a mean kinetic energy below $30 \mathrm{eV}$, the vast majority of the secondary electrons being below $70 \mathrm{eV}$. For each $\mathrm{MeV}$ of energy absorbed, about $3 \times 10^{4}$ low-energy electrons are created [132]. Secondary electrons travel further into the matter losing energy by scattering and new ionizations. The scattering events may sometimes be resonant, resulting in significant dissociative damage through the formation of transient negative ions (TNI) [133]. Each interaction reduces the electron kinetic energy, and eventually the electron will be trapped by thermalization, either in an empty non-dissociative molecular orbital creating a relatively stable molecular anion, or in some cases 'solvated' in a shallow intermolecular potential well, created by molecular dipoles (IMTE, see Sections 6.4 and 6.5). In solid state carbohydrates this will be two or more $\mathrm{OH}$ dipoles [134]. Both the oxidation products and the reduction products will normally be left in vibrationally and sometimes also electronically excited states. Relaxation of these states often provides the activation energy necessary for subsequent processes.

Solid state organic materials usually are very good insulators [135]: extensive charge transfer processes will not take place. This is the main reason why charge recombination is prohibited, radicals become trapped and detectable with EMR. Solid state carbohydrates often give a high yield of radicals at low temperatures, indicating that charge transfer reactions are rare. Of course charge transfer does take place under given conditions [21,136], but its range is limited and it enters in strong competition with radical trapping and subsequent secondary reactions.

\subsubsection{Primary Processes}

\section{Reduction}

The one-electron reduced radical species (radical anion) will either protonate, most often through a hydrogen bond, or the additional charge becomes eliminated by expelling a negatively charged fragment like $\mathrm{H}^{-}$or $\mathrm{OH}^{-}$(typically from TNI's by dissociative electron attachment). Such mechanisms physically separate charge and spin, thereby reducing the possibility for charge combination processes. $\mathrm{H}^{-}$ and $\mathrm{OH}^{-}$are reactive and may readily capture a proton, forming $\mathrm{H}_{2}$ or $\mathrm{H}_{2} \mathrm{O}$. 
Hydrogen bonding is associated with the hydroxyl substituents in carbohydrates and the initial reduction and protonation may be followed by dissociation of $\mathrm{H}$ atoms or $\mathrm{OH}$ radicals, which in turn may attack neighboring undamaged molecules, most often abstracting a hydrogen atom from these neighbors. High yields of hydrogen gas have been measured upon irradiation of carbohydrates [137].

The major results of the primary reductive trapping reactions in carbohydrates are hence neutral $\mathrm{C}$-centered radicals. The most common products identified, as evident from the Tables in Section 6.5, are neutral C-centered hydroxyalkyl radicals apparently formed by net $\mathrm{H}$-abstraction. It is experimentally not possible to distinguish between such initially reduced species and radicals formed by prompt $\mathrm{H}$-abstraction from molecules left in highly excited (superexcited) states. Due to the lack of thermal energy, usually only simple processes requiring a minimum of energy are considered as relevant for explaining the neutral C-centered hydroxyalkyl radicals observed at low temperatures.

Carbohydrates form a group of compounds in which electrons may become intermolecularly trapped, due to the large number of $\mathrm{OH}$ groups exhibiting large dipolar moments. These IMTE centers may be compared with solvated electrons in frozen solutions [138]. The IMTE may also be compared with a polaron [139], as the trapping of the electrons probably results in a reorientation of $\mathrm{OH}$ fragments due to the excess electronic charge, enhancing the propensity for temporary trapping and consequent screening of the excess charge. The electron wave function is spread over a number of hydroxyl protons (in the vicinity of the trapping site), which in turn are somewhat displaced from their equilibrium positions. Upon thermal activation or exposure to visible light, the IMTE is excited and transfers to a neighboring $\mathrm{OH}$ group initiating the formation of a $\mathrm{C}$-centered hydroxyalkyl radical as described by Eq. (6.9). It has been observed that optical and thermal bleaching can result in different products [140]. Lund and co-workers [134] have characterized the optical properties of trapped electrons for several carbohydrates and nicely demonstrated a well-developed absorption band in the $450-550 \mathrm{~nm}$ region.

The tables in Section 6.5 illustrate that radical formation is selective: only a few specific radicals appear to be formed from the vast number of possible species. The origin of this specificity is in principle unknown, but the observations indicate that primary radical formation is governed by overall energetic considerations, tightly associated with structural features of the compounds in question [21].

\section{Oxidation}

The one-electron oxidized species (radical cation) will, in analogy with the radical anion, eliminate the excess positive charge by expelling a proton. It appears likely that the $\mathrm{R}-\mathrm{OH}^{+}$is a general oxidation product [84]. This product deprotonates im- 
mediately, most often by way of the hydrogen bonding system. Under favorable conditions a proton will be transferred from an $\mathrm{OH}^{+}$group of the cation to a neighboring molecule, leaving behind an O-centered neutral alkoxy radical. As the hydrogen bonding scheme often is a continuous chain of O-bonded protons $(\mathrm{C}-\mathrm{OH}$ and/or $\mathrm{H}_{2} \mathrm{O}$ ) throughout the lattice, the deprotonation has in some cases been shown to initiate a proton shuffle covering several molecules in the lattice [20, 21]. The driving force for the deprotonation may partly result from a change in the acid-base equilibrium for the cation as compared with the neutral molecule [141] and the excitation energy available by vibrational relaxation of the initial oxidation product. This, in combination with the structural features of the environment is expected to explain the selectivity of the alkoxy radical formation.

If deprotonation at an $\mathrm{OH}$-group is not feasible (or back-protonation is immediate), it may rather occur at the neighboring carbon atom, resulting in a neutral $\mathrm{C}$ centered species [84]. Highly excited oxidative states created by the ionization event may in addition produce a number of other radical or non-radical fragments [142].

The species formed by one-electron oxidation/reduction or from super-excited states, as described here, are those commonly observed at the lowest irradiation temperatures. The mechanisms are based mainly on radiation physical/chemical principles, and the diversity of species from each of the compounds investigated illustrates the delicate overall energy balance of the system, whose structural properties are essential. It is therefore not surprising to observe several reduction and oxidation products simultaneously, even at the lowest irradiation temperatures. It is remarkable that in spite of the tremendous progress made in the field of molecular modeling, to date no modeling routines have been presented yet for reliably predicting the outcomes of radiation action at low temperatures.

\subsubsection{Secondary Processes}

Once the primary neutral radicals have been formed and trapped in the solid lattice, and thermal energy is supplied to the system, secondary processes take over the radical formation development. Here, thermally activated electronic reorganization processes and bond scissions dominate. Consequently, understanding the processes critically relies on the possibility to follow the transitions which occur in detail. Radiation chemists have investigated radical chemistry for years, but almost exclusively in solution, and occasionally in frozen solutions and glasses [125]. In crystalline solids, however, the conditions are basically different. Generally, only few mechanistic approaches are available for these systems, and intramolecular conversions are the most probable ones. Experimentally, each conversion between radical species must be followed sufficiently closely to convincingly argue for their interrelationship. 


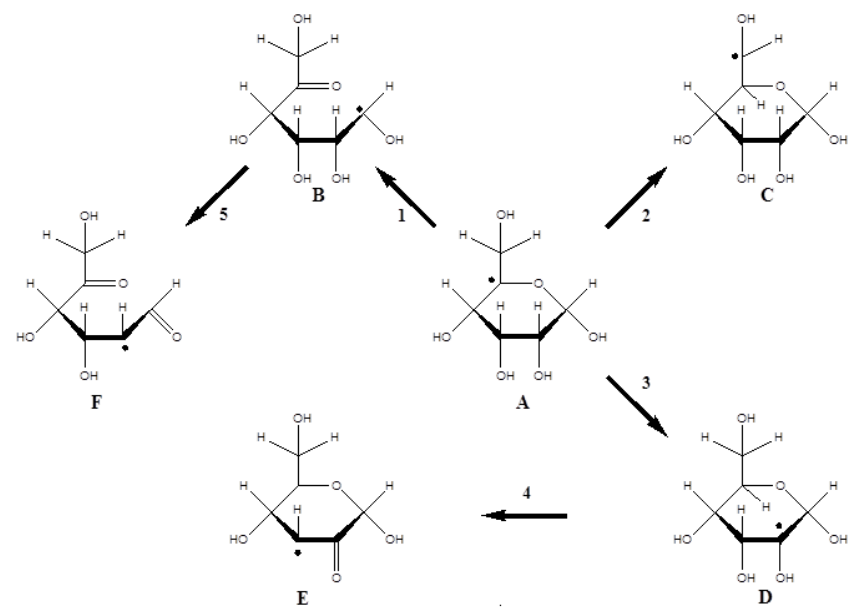

Fig. 6.17. Starting from a given primary radical structure A, processes $1-5$ illustrate electronic reorganization (1), 1,2 $\mathrm{H}$-atom shift (2) 1,4 $\mathrm{H}$-atom shift (3), and $\beta$-OH elimination (4 and 5) processes, the latter with the release of a $\mathrm{H}_{2} \mathrm{O}$ molecule.

In carbohydrates, there are generally no prominent leaving groups present, as one finds in e.g. amino acids (amino group, carboxyl group). Starting from the neutral reduction and oxidation products, Fig. 6.17 schematically illustrates the following possible processes that have been argued to occur in the solid state (among a few others): 1 - thermally induced electronic reorganization; 2 and 3 common 1,2- and 1,4 $\mathrm{H}$-atom shifts; 4 and 5 - elimination reactions like $\beta$-OH elimination. Reactions $1+5(\mathrm{~A} \rightarrow \mathrm{B} \rightarrow \mathrm{F})$ demonstrate how relatively simple processes may severely alter the molecular structure.

\subsubsection{Radical reactions}

\section{Sucrose}

After LT irradiation 4 major radicals were observed: the C1(-H), C5(-H), C6(-H) and the O3' alkoxy radical (Section 6.4.2). An IMTE was identified, shown to transfer to the $\mathrm{C} 5(-\mathrm{H})$ radical by optical excitation or careful warming in the dark $[104,109]$. A C6(-H) $\rightarrow$ C5 $(-\mathrm{H})$ transformation was very probably observed upon annealing [104], which may be explained as a (C5,C6) $\mathrm{H}$-shift reaction in the opposite direction of process 2 in Fig. 6.17. The neutral $\mathrm{C} 6(-\mathrm{H})$ may either be a primary oxidation product formed by deprotonation at $\mathrm{C} 6$ of the $\mathrm{RCH}_{2} \mathrm{OH}^{+}$species [84], or alternatively be formed by a net $\mathrm{H}$-abstraction in a reductive process. The $\mathrm{C} 1(-\mathrm{H})$ radical was tentatively ascribed to oxidative deprotonation, based on stoichiometric considerations that assume $\mathrm{C} 6(-\mathrm{H})$ to be a reduction species. 
The O3' alkoxy radical decays in the 50-80 K range. Again, a (C3',O3') Hshift (or back-protonation followed by a deprotonation from C3') will create a C3' centered intermediate, which by a $\beta-\mathrm{OH}$ elimination may yield a radical centered at $\mathrm{C} 4$ '. From here it is possible to speculate that a $\mathrm{C}^{\prime}{ }^{\prime}(-\mathrm{H})$ radical species may become stabilized, the most probable site for the intermediate radical N1. More detailed EMR and DFT characterization of N1 and the two as yet unidentified species at $80 \mathrm{~K}$ ( $\mathrm{N} 2$ and $\mathrm{N} 3$, not formed by simple H-abstractions) is necessary to elucidate the fate of the alkoxy species. $\mathrm{C} 5(-\mathrm{H})$ and $\mathrm{C} 1(-\mathrm{H})$ also remain as dominant paramagnetic species at $80 \mathrm{~K}$. The precursors for the RT radicals are expected to be among these five species.

Above $100 \mathrm{~K}$, the $\mathrm{C} 5(-\mathrm{H})$ radical disappears, and above $200 \mathrm{~K}$ also $\mathrm{C} 1(-\mathrm{H})$. The RT stable radicals $\mathrm{T} 1$ and $\mathrm{T} 2 / \mathrm{T} 3$ are also present immediately after irradiation at RT, along with $\mathrm{U} 1$ and $\mathrm{U} 2$. It was proposed that the $\mathrm{C} 1(-\mathrm{H})$ radical may be the precursor for the stable radical T2/T3, proceeding through a $(\mathrm{C} 5, \mathrm{C} 6) \mathrm{H}$-shift followed by an elimination reaction. With regard to the $\mathrm{C} 5(-\mathrm{H})$ radical, an electronic reorganization may be suggested that leaves the spin at $\mathrm{C} 1$, and a subsequent elimination reaction could lead to the T1 radical. However, the RT unstable U1 and U2 species, which have not been identified yet and seem to evolve in diamagnetic radiation products, may also result from the decay of $\mathrm{C} 5(-\mathrm{H})$ and/or $\mathrm{C} 1(-\mathrm{H})$. Therefore, the picture is still confusing with regard to processes between $100 \mathrm{~K}$ and RT. Work in progress towards identification of U1 and U2 will hopefully clear out this situation.

The present discussion clearly shows that, even if a vast amount of information on the radiation responses of sucrose is available (more than for any other carbohydrate to date), mapping specific radical reaction mechanisms remains to some extent speculative. Time-consuming and tedious monitoring the conversion of each component into its successor is necessary. This is work mostly remains to be done.

\section{K2G1P}

Glucose-phosphate is an interesting substituted carbohydrate: a phosphate group is linked to a carbohydrate unit, mimicking the (deoxy)ribonucleotide ester bond in nucleic acids. A number of studies on K2G1P crystals have been summarized in Section 6.5.2 (Table 6.10, [11, 88, 103, 115, 118, 119]).

The LT irradiation study demonstrated that 3 different alkoxy radicals (A1A3), an IMTE and a C6(-H) (labeled IV) hydroxyalkyl radical are trapped [88]. As no thermal or optical bleaching studies were reported, details on the processes connecting these radicals to those stable at elevated temperatures are not available from experiment. No P-centered radicals were observed after LT irradiation. 
At $80 \mathrm{~K}$, the 4 dominating radicals were all hydroxyalkyl radicals, labeled R1R4 in Table 6.10. R3 and R4 are two distinct geometrical conformations of the $\mathrm{C} 6(-\mathrm{H})$ radical, also detected after LT irradiation ( $\mathrm{IV}=\mathrm{R} 4)$. Conformationally different $\mathrm{C} 6(-\mathrm{H})$ radicals may be formed from the $\mathrm{O} 6$ alkoxy radical A1, e.g. by back-transfer of the proton and consecutive deprotonation at C6 [84], or by a Hshift process. Similarly, H-atoms released by the decay of other LT-trapped radicals may lead to $\mathrm{C} 6(-\mathrm{H})$ radicals.

$\mathrm{R} 1$ is the $\mathrm{C} 3(-\mathrm{H})$ radical species and good agreement with experimental data in the DFT modeling was only obtained if one assumes that the abstracted proton is transferred to a neighboring phosphate group [11]. This was hypothesized to be the 'missing' oxidation product at $\mathrm{C} 3$ : rather than an $\mathrm{O} 3(-\mathrm{H})$ alkoxy, the $\mathrm{C} 3(-\mathrm{H})$ radical is produced [84]. This model remains to be confirmed by analyzing hereto non-analyzed components of the spectra obtained after LT irradiation [88].

$\mathrm{R} 2$ is a $\mathrm{C} 1$-centered species formed by the net scission of the phosphate ester bond. Its structure is similar to that of T2/T3 in sucrose. A model for the formation of R2 was proposed [11], starting from a tentative $\mathrm{C} 2(-\mathrm{H})$ hydroxyalkyl radical and proceeding by $\beta$-phosphate elimination, leaving the unpaired spin at $\mathrm{C} 1$, in addition to a non-radical $\mathrm{HOPO}_{2}{ }^{2-}$ species. The $\mathrm{C} 2(-\mathrm{H})$ species may be formed from the $\mathrm{O} 2$ alkoxy radical $(\mathrm{A} 3)$ by a $(\mathrm{C} 2, \mathrm{O} 2) \mathrm{H}$-atom shift.

Other radicals were detected at $80 \mathrm{~K}$ but not identified [103, 116]. None of these appeared to be alkoxy radicals, IMTE's or phosphate-centered radicals. At $\mathrm{RT}$, the only well-identified species is a phosphoryl $\mathrm{PO}_{3}{ }^{2-}$ radical [119]. A proposed mechanism for the formation of this radical [119] was however questioned in ref. [11] on basis of its large endothermicity. Other processes, e.g. electronic reorganization from a $\mathrm{C} 1(-\mathrm{H})$ species, may be envisioned for the formation of a phosphoryl radical, but without more detailed investigation of the $\mathrm{g}=2$ products at RT and the connections between radical products occurring between $80 \mathrm{~K}$ and $\mathrm{RT}$, any suggestion remains highly speculative.

\section{Trehalose dihydrate}

Table 6.11 reveals a very complex and in part confusing array of radicals formed by irradiation at various temperatures. Based on our recent EMR/DFT work [12, $20,101]$ many early radical assignments $[4,111,120]$ needed to be corrected. However, it was not always evident to establish the correspondence with these earlier publications unambiguously. A considerable number of proposed radical models are based on few data, leaving some identifications rather uncertain.

The situation after LT irradiation appears rather clear, with the formation of an IMTE center (not seen by Tarpan et al. [20]), two alkoxy radicals (probably at $\mathrm{O} 2$ and O4', Samskog and Kispert report only the latter [111]) and two hydroxyalkyl radicals (identified as $\mathrm{C} 5(-\mathrm{H})$ and $\mathrm{C}^{\prime}(-\mathrm{H})$, Samskog and Kispert report only the 
latter [111]). Furthermore, it was noted [111] that the intensity of C-centered radical(s) increased after thermal annealing to $50 \mathrm{~K}$ and was stable at $77 \mathrm{~K}$. The fact that Tarpan et al. did not observe the IMTE at slightly higher irradiation temperature, but did detect an extra $\mathrm{C} 5$-centered radical, might imply a thermal transformation from the former to the latter radical at a relatively low temperature. On the other hand, the reasons for not detecting the IMTE at X-band [20] may be similar as for sucrose (see Section 6.4.2). Ref. [20] reports another interesting feature of the O4' alkoxy radical: in order to reproduce the experimental data in the DFT modeling, the abstracted proton needed to be shuffled through three hydrogen bonds and trapped as a hydronium ion. A similar observation was made for an alkoxy radical in crystals of rhamnose $[17,21]$.

After $77 \mathrm{~K}(80 \mathrm{~K})$ irradiation, the situation looks considerably more complicated. Both Samskog et al. [120] and Tarpan et al. [101] reported four alkoxy and three hydroxyalkyl radicals, but two radicals in each study $\left(\mathrm{V}^{77 \mathrm{~K}}, \mathrm{VI}^{77 \mathrm{~K}} / \mathrm{T} 2, \mathrm{~T} 3\right)$ have no clear counterpart in the other. In addition, only for two radicals DFT modeling allowed convincing (certain) identification, and one of these, $\mathrm{C} 5(-\mathrm{H})$ (R2) is already present after $10 \mathrm{~K}$ irradiation. Also one of the alkoxy species created at $10 \mathrm{~K}$ remains stable up to $80 \mathrm{~K}(\mathrm{O} 2(-\mathrm{H}))$. The other alkoxy, the IMTE and the $\mathrm{C}^{\prime}(-\mathrm{H})$ radical decay in the in the $10-80 \mathrm{~K}$ temperature range [101] and may be expected to transform into 6 new paramagnetic species. Indeed, pulse annealing in the 10-80 K range [101] showed (almost perfect) equivalence between LT irradiation followed by annealing to $80 \mathrm{~K}$ and irradiation at the latter temperature.

The fact that irradiation at $77 \mathrm{~K}$ yields a larger variety of alkoxy species than irradiation at LT is remarkable. It is possible that the larger thermal energy available at $77 \mathrm{~K}$ would allow for a larger diversity in alkoxy formation from the primary cation radical. Studying the thermal annealing [101] of samples irradiated at LT in further detail may bring answers to this question. Concerning the hydroxyalkyl radicals, for $\mathrm{T} 1$, dominant at $80 \mathrm{~K}$ and identified as $\mathrm{C} 4(-\mathrm{H})$ (certain), it is not evident to construct a reasonable reaction chain starting from the radicals identified after LT irradiation. Furthermore it is not straightforward to link the unstable alkoxy $\left(\mathrm{O}^{\prime}(-\mathrm{H})\right)$ to any of the hydroxyalkyl radicals at $80 \mathrm{~K}$. Tarpan et al. [101] argue that in spite of the structural information available, it is still premature to speculate on mechanisms, as the actual conversions have not been followed in detail. Indeed, it is possible that the decay of LT radicals results in diamagnetic molecules and $\mathrm{H}$-atoms (or other mobile radical fragments), and that the action of the latter is to a large extent unpredictable without further experimental information. The large decrease in EPR intensity observed in the 10-80 K pulse annealing experiments [81], indeed, points to formation of diamagnetic radiation damage.

The RT studies [4, 12] agree on the presence of a dominant EPR triplet contribution immediately after irradiation, I1, identified as $\mathrm{C} 3{ }^{\prime}(-\mathrm{H})$, and two contributions to the final stable spectrum ( $\mathrm{S} 1$ and $\mathrm{S} 2$, only suggested models, no convincing identification). Tarpan et al. [12] detected two more species immediately after 
irradiation: one was identified as $\mathrm{C} 2(-\mathrm{H})$ with a $\beta$-carbonyl at $\mathrm{C} 3$, the other only has very limited stability and remained unidentified. Pulse annealing experiments between $80 \mathrm{~K}$ and RT have only been performed coarsely. From $150 \mathrm{~K}$ onwards, no strong changes seem to occur in the EPR spectrum (dominant triplet contribution). However, this may be deceiving as ENDOR analyses at $10 \mathrm{~K}, 80 \mathrm{~K}$ and RT have shown that three very similar EPR triplets correspond to hydroxyalkyl radicals centered at different C-atoms [12] (Table 6.11, $\mathrm{II}^{3 \mathrm{~K}}=\mathrm{R} 1, \mathrm{VII}^{77 \mathrm{~K}}=\mathrm{T} 1 / \mathrm{T} 1 *$, I1). Pulse annealing experiments evidenced a further decrease in the total EPR intensity, suggesting formation of diamagnetic species [81]. This hampers devising chemical links between the species observed immediately after RT irradiation and radicals stable at $80 \mathrm{~K}$. Gräslund and Löfroth [4] proposed $\beta$-elimination reactions to explain the transformations at RT to the final stable stage. The model suggested for S2 (C2(-H) with $\beta$-carbonyl at $\mathrm{C} 1)$ seems compatible with such hypothesis. The role of crystal water in this carbohydrate also deserves further investigation.

\section{Fructose}

The LT study (10 K irradiation) by Tarpan et al. [18] gave evidence for two hydroxyalkyl radicals: a $\mathrm{C} 3(-\mathrm{H})$ (certain) and a $\mathrm{C} 5(-\mathrm{H})$ species (plausible). Trapped electrons were not detected and no attempts were made to analyze weak features, probably due to alkoxy radicals. After irradiation at $80 \mathrm{~K}, \mathrm{C} 3(-\mathrm{H})$ species was still observed [91], but $\mathrm{C} 5(-\mathrm{H})$ was not. At this temperature, however, another radical was seen, identified (certain) as a ring-opened species with the spin localized at $\mathrm{C} 3$ (R2/R3). It was originally proposed that this ring-opened species originated from an $\mathrm{O} 2(-\mathrm{H})$ alkoxy radical [81]. Because at $10 \mathrm{~K}$ no alkoxy radical was observed as a dominant radiation defect, and the $\mathrm{C} 5(-\mathrm{H})$ hydroxyalkyl radical decayed between $10 \mathrm{~K}$ and $80 \mathrm{~K}$, a link between $\mathrm{C} 5(-\mathrm{H})$ and the ring-opened species seems more reasonable. However, if both structure assignments are correct, there is no straightforward reaction pathway between them. It may be speculated that $\mathrm{C} 5(-\mathrm{H})$ decays by releasing a $\mathrm{H}$-atom, which abstracts the $\mathrm{O} 2$ hydroxyl proton at a neighboring molecule, yielding the ring-opened species $\mathrm{R} 2 / \mathrm{R} 3$ by a subsequent electronic reorganization.

Thermal annealing at $160 \mathrm{~K}$ of crystals irradiated at $77 \mathrm{~K}$ resulted in two major radicals only. There is an apparent transformation of the $\mathrm{C} 3(-\mathrm{H})$ radical into a new species assigned to a $\mathrm{Cl}(-\mathrm{H})$ radical (plausible) [81]. The ring-opened species remained present at this temperature. Again, there is no obvious reaction pathway between the $\mathrm{C} 3(-\mathrm{H})$ and the $\mathrm{C} 1(-\mathrm{H})$ species, except for a $\mathrm{H}$-transfer process.

Upon irradiation of fructose crystals at RT followed by immediate cooling, unstable species were stabilized for a sufficiently long time to allow a detailed analysis [81]. Six distinct species (Im1-Im6, see Table 6.12) were detected and analyzed. Two of these remained stable upon storage at RT ( $\operatorname{Im} 2=$ F1/F2 and Im6 $=\mathrm{F} 4 / \mathrm{F} 5)$. The other 4 either decayed or developed into to further stable radicals 
F3 and F6 Table 6.12). None of these species had been observed in the studies at lower temperatures. Hence, a considerable number of reactions and rearrangements must occur between $160 \mathrm{~K}$ and $\mathrm{RT}$ and there is by far insufficient information presently available to provide direct links between these species. A few speculations can, nonetheless, be made. Indeed, e.g., the Im4 radical (plausible) can be formed from the $\mathrm{C} 1(-\mathrm{H})$ radical (plausible) by a simple $\beta-\mathrm{OH}$ elimination. The ring-opened species Q2, stable at least to $160 \mathrm{~K}$, most probably dehydrogenates, yielding one or several of the still unidentified RT radicals with limited stability, or even the stable radicals F1/F2, whose structure could not be completely elucidated. More firm conclusions will only be possible after closely monitoring which species are formed at stages between $160 \mathrm{~K}$ and RT.

\subsubsection{Final remarks}

In addition to the carbohydrates discussed above, a variety of other solid-state sugars have been investigated for different purposes. Box and co-workers have characterized IMTEs in a number of compounds, as nicely summarized in the review by Lund and Schlick [134]. The group of Box has characterized an even larger number of primary alkoxy radicals in carbohydrates [107-109, 144]. These authors, however, seldom discussed secondary radicals and radical reactions. Lund and coworkers have analyzed many different solid-state carbohydrates using both pulse radiolysis and EMR techniques. Some of these studies have been discussed above $[85,111,112,120]$. They elucidated several of the most important secondary reactions for IMTE and alkoxy radicals. Bernhard and co-workers [22, 143] investigated some carbohydrate derivatives in large detail, and also in some cases they provided possible reaction sequences. The carbohydrate rhamnose has been studied by several groups, both for characterizing IMTE and alkoxy radicals, and for discussing reaction sequences ([140] and references therein). Advanced DFT calculations for this compound have yielded important information on oxidative proton shuffling at low temperatures $([17,21]$, see also Chapter 18 of this book). Presently, theoretical and experimental work, predicting the initial radical products after ionizing radiation as well as providing detailed understanding of the nature and reactions of the IMTE and alkoxy radicals, is ongoing [145]. This also includes mapping of radical reactions from C-centered low temperature precursors into RT stable radicals. 


\subsection{Summary and conclusions}

In this chapter, we have outlined the strategy and reviewed the results of our EMR studies of radiation-induced radicals in solid-state sucrose, dipotassium glucose-1phosphate, trehalose dihydrate, fructose and sorbose. This work has been primarily directed towards identification of radical structures. We have shown that single crystal EPR, ENDOR and EIE measurements, together with DFT modeling, present a winning combination for radical identification. Comparing $\mathrm{SH}$ parameters resulting from carefully analyzed experiments (e.g. eliminating the Schonland ambiguity) with high-level DFT calculations on correct radical models can lead to remarkably good agreement. This holds particularly true for the $g$ and $A$ tensor principal directions and the anisotropy in the principal values, but also isotropic values are fairly well predicted by calculations.

For most carbohydrates discussed here, radicals have been identified after irradiation at various temperatures, in order to follow the reaction pathways between the primary radiation products and the final stable radicals. Low-temperature irradiation $(4-10 \mathrm{~K})$, as expected, produces IMTE centers along with net $\mathrm{H}$-abstracted alkoxy and hydroxyalkyl radicals. The stable radiation-induced radicals are Ccentered and often feature carbonyl formation close to the radical center, evidenced by an increased anisotropy of the $g$ tensor. The multi-compositeness of the spectra, that in addition considerably lose intensity upon annealing after irradiation at low temperature, renders direct monitoring of inter-conversions between radical species difficult. Hence, despite the experimental and modeling efforts, for none of the carbohydrates discussed here, the radiation chemistry could be elucidated. Nonetheless, certain plausible reaction paths have been presented and considerable progress is currently being made for other carbohydrates.

In the context of applying the EPR spectra of sugars for dosimetry and detection of irradiated foodstuffs, we recently made significant progress in understanding the EPR spectrum of irradiated sucrose powder. Our studies provide a mechanistic understanding of the common practice to wait at least 48 hours (allowing for the U1 and U2 components to decay) before using the spectrum of irradiated sucrose for dosimetric purposes. Simulations taking into account the contributions of the three stable radicals, that were thoroughly characterized using single crystal EMR measurements and identified via DFT modeling, reproduce the central part of this spectrum very well. The stable radicals responsible for the remaining discrepancies are currently being characterized. Somewhat surprisingly, the knowledge of the radical models also appears helpful for powder EPR spectrum simulations.

Acknowledgments The authors thank the many $\mathrm{PhD}$ and Master students whose experimental work has contributed to this chapter, which we dedicate to the memories of Profs. W.H. Nelson and W.A. Bernhard. 
1. Ueda H, Kuri Z, Shida S (1961) Electron Spin Resonance Studies of Gamma-Irradiated Single Crystals of Sucrose. J Chem Phys 35:2145.

2. Shields H, Hamrick P (1962) X-Irradiation Damage of Sucrose Single Crystal. J Chem Phys 37: 202-203.

3. Lomaglio G (1967) Résonance Paramagnétique Electronique et Susceptibilité Paramagnétique d'un Monocristal de Saccharose Irradié. C R Séances Acad Sci Ser B 264: 1637.

4. Gräslund A, Löfroth G (1975) Free-Radicals in Gamma-Irradiated Single-Crystals of Trehalose Dihydrate and Sucrose Studied by Electron-Paramagnetic Resonance. Acta Chem Scand Ser B 29: 475-482.

5. Sagstuen E, Lund A, Awadelkarim O, Lindgren M, Westerling J (1986) Free-Radicals in X-Irradiated Single-Crystals of Sucrose - A Reexamination. J Phys Chem 90: 5584-5588.

6. Vanhaelewyn G, Sadlo J, Callens F, Mondelaers W, De Frenne D, Matthys P (2000) A Decomposition Study of the EPR Spectrum of Irradiated Sucrose. Appl Radiat Isot 52:1221-1227.

7. Georgieva ER, Pardi L, Jeschke G, Gatteschi D, Sorace L, Yordanov ND (2006) HighField/High-Frequency EPR Study on Stable Free Radicals Formed in Sucrose by GammaIrradiation. Free Radical Res 40:553-563.

8. De Cooman H, Pauwels E, Vrielinck H, Sagstuen E, Callens F, Waroquier M (2008) Identification and Conformational Study of Stable Radiation-Induced Defects in Sucrose Single Crystals Using Density Functional Theory Calculations of Electron Magnetic Resonance Parameters. J Phys Chem B 112:7298-7307.

9. De Cooman H, Pauwels E, Vrielinck H, Sagstuen E, Van Doorslaer S, Callens F, Waroquier M (2009) ENDOR and HYSCORE Analysis and DFT-Assisted Identification of the Third Major Stable Radical in Sucrose Single Crystals X-Irradiated at Room Temperature. Phys Chem Chem Phys 11:1105-1114.

10. Vanhaelewyn GCAM, Jansen B, Pauwels E, Sagstuen E, Waroquier M, Callens FJ (2004) Experimental and Theoretical Electron Magnetic Resonance Study on Radiation-Induced Radicals in Alpha-L-Sorbose Single Crystals. J Phys Chem A 108:3308-3314.

11. Pauwels E, De Cooman H, Vanhaelewyn G, Sagstuen E, Callens F, Waroquier M (2008) Radiation-Induced Radicals in Glucose-1-Phosphate. II. DFT Analysis of Structures and Possible Formation Mechanisms. J Phys Chem B 112:15054-15063.

12. De Cooman H, Tarpan MA, Vrielinck H, Waroquier M, Callens F (2013) Room Temperature Radiation Products in Trehalose Single Crystals: EMR and DFT Analysis. Radiat Res 179:313-322.

13. Tarpan MA, Vrielinck H, De Cooman H, Callens F (2009) Determination of the $g$ Tensors for the Dominant Stable Radicals in X-Irradiated Beta-D-Fructose Single Crystals. J Phys Chem A 113:7994-8000.

14. Tarpan MA, Pauwels E, Vrielinck H, Waroquier M, Callens F (2010) Electron Magnetic Resonance and Density Functional Theory Study of Room Temperature X-Irradiated BetaD-Fructose Single Crystals. J Phys Chem A 114:12417-12426.

15. Box HC (1977) Radiation Effects: ESR and ENDOR analysis. Academic Press, New York.

16. Pauwels E, Van Speybroeck V, Waroquier M (2006) Radiation-Induced Radicals in Alpha-D-Glucose: Comparing DFT Cluster Calculations with Magnetic Resonance Experiments. Spectrochim Acta A 63:795-801.

17. Pauwels E, Declerck R, Van Speybroeck V, Waroquier M (2008) Evidence for a Grotthuss-Like Mechanism in the Formation of the Rhamnose Alkoxy Radical Based on Periodic DFT Calculations. Radiat Res 169:8-18.

18. Tarpan M, Sagstuen E, Pauwels E, Vrielinck H, Waroquier M, Callens F (2008) Combined Electron Magnetic Resonance and Density Functional Theory Study of $10 \mathrm{~K}$ X-Irradiated Beta-D-Fructose Single Crystals. J Phys Chem A 112:3898-3905. 
19. De Cooman H, Pauwels E, Vrielinck H, Sagstuen, E, Waroquier M, Callens F (2010) Oxidation and Reduction Products of X Irradiation at $10 \mathrm{~K}$ in Sucrose Single Crystals: Radical Identification by EPR, ENDOR, and DFT. J Phys Chem B 114:666-674.

20. Tarpan MA, De Cooman H, Sagstuen E, Waroquier M, Callens F (2011) Identification of Primary Free Radicals in Trehalose Dihydrate Single Crystals X-irradiated at $10 \mathrm{~K}$. Phys Chem Chem Phys 13:11294-11302.

21. Aalbergsjö SG, Pauwels E, De Cooman H, Hole EO, Sagstuen E (2013) Structural Specificity of Alkoxy Radical Formation in Crystalline Carbohydrates. Phys Chem Chem Phys 15:9615-9619.

22. Madden KP, Bernhard WA (1979) ESR-ENDOR study of $\alpha$-D-Glucopyranose Single Crystals X Irradiated at 12 and $77 \mathrm{~K}$. J Phys Chem 83:2643-2649.

23. Von Sonntag C (2006) Free-Radical-Induced DNA Damage and its Repair - A Chemical Perspective. Springer-Verlag, Berlin.

24. Nakajima T (1988) Sugar as an Emergency Populace Dosimeter for Radiation Accidents. Health Phys 55:951-955.

25. Nakajima T (1989) Possibility of Retrospective Dosimetry for Persons Accidentally Exposed to Ionizing-Radiation Using Electron-Spin Resonance of Sugar and Mother-ofPearl. Br J Radiol 62:148-153.

26. Silveira FAM, Baffa O (1995) Lyoluminescence and ESR Measurements on Alanine and Sucrose Dosimeters. Appl Radiat Isot 46:827-830.

27. Son PK, Ok CI, Kim JW (2001) EPR Study of Sugar Irradiated with X-Rays. J Korean Phys Soc 38:315-317.

28. Yordanov ND, Gancheva V, Georgieva E (2002) EPR and UV Spectroscopic Study of Table Sugar as a High-Dose Dosimeter. Radiat Phys Chem 65:269-276.

29. Yordanov ND, Georgieva E (2004) EPR and UV Spectral Study of Gamma-Irradiated White and Burned Sugar, Fructose and Glucose. Spectrochim Acta A 60:1307-1314.

30. Desrosiers M, Wadley S (2006) Time Dependence of the Radiation-Induced EPR Signal in Sucrose. Radiat Prot Dosim 118:479-481.

31. Trompier F, Bassinet C, Wieser A, De Angelis C, Viscomi D, Fattibene P (2009) Radiation-Induced Signals Analysed by EPR Spectrometry Applied to Fortuitous Dosimetry. Ann Ist Super Sanita 45:287-296.

32. Karakirova Y, Yordanov ND, De Cooman H, Vrielinck H, Callens F (2010) Dosimetric Characteristics of Different Types of Saccharides: An EPR and UV Spectrometric Study. Radiat Phys Chem 79:654-659.

33. Desrosiers MF (1996) Current Status of the EPR Method to Detect Irradiated Food. Appl Radiat Isot 47:1621-1628.

34. European Committee for Standardisation (2001) EN 13708:2001 E: Foodstuffs - Detection of Irradiated Food Containing Crystalline Sugar by ESR Spectroscopy. CEN, Brussels.

35. Malec-Czechowska K, Strzelczak G, Dancewicz AM, Stachowicz W, Delincée H (2002) Detection of Irradiation Treatment in Dried Mushrooms by Photostimulated Luminescence, EPR Spectroscopy and Thermoluminescence Measurements. Eur Food Res Technol 216:157-165.

36. Yordanov ND, Pachova Z (2006) Gamma-Irradiated Dry Fruits - An Example of a Wide Variety of Long-Time Dependent EPR Spectra. Spectrochim Acta A 63:891-895.

37. Guzik GP, Stachowicz W, Michalik J (2008) Study on Stable Radicals Produced by Ionizing Radiation in Dried Fruits and Related Sugars by Electron Paramagnetic Resonance Spectro-metry and Photostimulated Luminescence Method - I. D-fructose. Nukleonika 53:S89-S94.

38. Guzik GP, Stachowicz W, Michalik J (2012) EPR Study on Sugar Radicals Utilized for Detection of Radiation Treatment of Food. Nukleonika 57:545-549.

39. Ahn JJ, Akram K, Kwon JH (2012) Electron Spin Resonance Analyses of Grinding- and Radiation-Induced Signals in Raw and Refined Sugars. Food Anal Methods 5:1196-1204. 
40. Son PK, Choi S-W, Kim SS, Gwag JS (2012) Dosimetry Application of Irradiated DFructose Using the Electron Paramagnetic Resonance. J Magnetics 17:271-274.

41. Ahn J-J, Akram K, Kim H-K, Kwon J-H (2013) Electron Spin Resonance Spectroscopy for the Identification of Irradiated Foods with Complex ESR Signals. Food Anal Methods 6:301-308.

42. Mangiacotti M, Marchesani G, Floridi F, Siragusa G, Chiaravalle AE (2013) Official Checks by an Accredited Laboratory on Irradiated Foods at an Italian Market. Food Control 33:307-312.

43. Schonland DS (1959) On the Determination of the Principal g-Values in Electron Spin Resonance. Proc Phys Soc London 73:788-792.

44. Vrielinck H, De Cooman H, Tarpan MA, Sagstuen E, Waroquier M, Callens F (2008) Schonland Ambiguity in the Electron Nuclear Double Resonance Analysis of Hyperfine Interactions: Principles and Practice. J Magn Reson 195:196-205.

45. Nelson, W. H. (1980) Quick-Access Sample System for Low-Temperature ESR-ENDOR at K-Band. J Magn Reson 37:205-207.

46. Krzystek J, Sienkiewicz A, Pardi L, Brunel (1996) DPPH as a Standard for High-Field EPR. J Magn Reson 125:207-211.

47. Serway RA, Marshall SA (1967) Electron Spin Resonance Absorption Spectra of $\mathrm{CO}_{3}{ }^{-}$ and $\mathrm{CO}_{3}{ }^{3-}$ Molecule-Ions in Irradiated Single-Crystal Calcite. J Chem Phys 46:1949-1952.

48. Schweiger A, Jeschke G (2001) Principles of Pulsed Electron Paramagnetic Resonance. Oxford University Press, Oxford.

49. Weil JA, Bolton JR, Wertz, JE (1994) Electron Paramagnetic Resonance - Elementary Theory and Practical Applications. John Wiley \& Sons, New York.

50. Loncke F, De Cooman H, Khaidukov NM, Vrielinck H, Goovaerts E, Matthys P, Callens F (2007) EPR and ENDOR Analysis of $\mathrm{Fe}^{3+}$ Impurity Centers in Fluoroelpasolite Lattices. Phys Chem Chem Phys 9:5320-5329.

51. Vrielinck H, De Cooman H, Karakirova Y, Yordanov ND, Callens F (2009) Early-Stage Evolution of the EPR Spectrum of Crystalline Sucrose at Room Temperature after HighDose X Irradiation. Radiat Res 172:226-233.

52. Hyde JS (1965) ENDOR of Free Radicals in Solution. J Chem Phys 43:1806.

53. Robinson BH, Dalton LA, Beth AH, Dalton RL (1976) ENDOR Induced Electron Paramagnetic Resonance: Application to the Resolution of Overlapping Spectra. Chem Phys 18:321-332.

54. Andersen MF, Sagstuen E, Henriksen T (1987) Radiation Damage to Steroids. An ENDOR Study to Cholest-4-en-3-one. J Magn Reson 71:461-475.

55. De Cooman H, Pauwels E, Vrielinck H, Dimitrova A, Yordanov ND, Sagstuen E, Waroquier M, Callens F (2008) Radiation-Induced Defects in Sucrose Single Crystals, Revisited: A Combined Electron Magnetic Resonance and Density Functional Theory Study. Spectrochim Acta A 69:1372-1383.

56. Kusakovskij J, Vrielinck H, Callens F, work in progress.

57. De Cooman H, Keysabyl J, Kusakovskij J, Van Yperen-De Deyne A, Waroquier M, Callens F, Vrielinck H (2013) Dominant Stable Radicals in Irradiated Sucrose: $g$ Tensors and Contribution to the Powder Electron Paramagnetic Resonance Spectrum. J Phys Chem B 117:7169-7178.

58. Vrielinck H, Kusakovskij J, Vanhaelewyn G, Matthys P, Callens F (2013) Understanding the Dosimetric Powder EPR Spectrum of Sucrose by Identification of the Stable Radiation-Induced Radicals. Radiat Prot Dosim, submitted for publication.

59. Nelson WH (1980) Estimation of Errors in Eigenvalues and Eigenvectors from Magnetic Resonance Results by Use of Linear Data-Fitting Techniques. J Magn Reson 38:71-78.

60. Stoll S, Schweiger A (2006) EasySpin, a Comprehensive Software Package for Spectral Simulation and Analysis in EPR. J Magn Reson 178:42-55. 
61. Lund A, Thuomas KA, Maruani J (1978) Calculation of Powder ESR-Spectra of Radicals with Hyperfine and Quadrupolar Interactions - Application to Monochloroalkyl and Dichloroalkyl Radicals. J Magn Reson 30:505-514.

62. Sagstuen E, Lund A, Itagaki Y, Maruani J (2000) Weakly Coupled Proton Interactions in the Malonic Acid Radical: Single Crystal ENDOR Analysis and EPR Simulation at Microwave Saturation. J Phys Chem A 104:6362-6371.

63. Kang J, Tokdemir S, Shao J, Nelson WH (2003) Electronic g-Factor Measurement from ENDOR-Induced EPR Patterns: Malonic Acid and Guanine Hydrochloride Dihydrate. J Magn Reson 165:128-136.

64. Theisen H, Sagstuen E (1981) The Indole H-Adduct Radical in Single Crystals of Tryptamine-HCl: an ESR-ENDOR Study. J Chem Phys 74:2319-2324.

65. Sagstuen E, Awadelkarim O, Lund A, Masiakowski J (1986) Trapping Site Geometry of $\mathrm{N}_{2} \mathrm{H}_{4}^{+}$Radical Ion in X-Irradiated Single-Crystals of $\mathrm{N}_{2} \mathrm{H}_{5} \mathrm{HC}_{2} \mathrm{O}_{4}$ - an ENDOR Study. J Chem Phys 85:3223-3228.

66. Maes F (1996) Electron Paramagnetic Resonance and Electron Nuclear Double Resonance of Chalcogen Ions in Alkali Halide Single Crystals. PhD Thesis, Ghent University [in Dutch].

67. Dobbs AJ, Gilbert BC, Norman ROC (1971) Electron Spin Resonance Studies. XXVII. Geometry of Oxygen-Substituted Alkyl Radicals. J Chem Soc A 124-135.

68. Bernhard WA (1984) The Use of Alpha Hyperfine Coupling Tensors as a Measure of Unpaired Spin Density and Free Radical Geometry. J Chem Phys 81:5928-5936.

69. Muto H (1991) Trapped Anions in Organic Systems. in: Lund A, Shiotani M (Eds.) (1991) Radical Ionic Systems. Properties in Condensed Phases. Kluwer Academic Publishers, Dordrecht, pp. 337-360.

70.Sørnes AR, Sagstuen E (1995) ENDOR Study of ${ }^{1} \mathrm{H}$ Couplings in Single Crystals of 2Aminoethyl Hydrogen Sulfate X-Irradiated at 295 K. Radical Geometry Analysis Using Effective Dipole Center Approximations and UHF-INDO calculations. J Phys Chem 99:16857-16866

71. Colson AO, Sevilla MD (1995) Structure and Relative Stability of Deoxyribose Radicals in a Model DNA Backbone: Ab Initio Molecular Orbital Calculations. J Phys Chem 99:3867-3874.

72. Erling PA, Nelson WH (2004) Dependence of Alpha-Proton Hyperfine Couplings on Free Radical Geometry. J Phys Chem A 108:7591-7595.

73. Øhman KT, Sanderud A, Hole EO, Sagstuen E (2006) Single Crystals of L-O-Serine Phosphate X-Irradiated at Low Temperatures: EPR, ENDOR, EIE, and DFT Studies. J Phys Chem A 110:8585-9596.

74. McConnell HM, Strathdee J (1959) Theory of Anisotropic Hyperfine Interactions in PiElectron Radicals. Mol Phys 2:129-138.

75. McConnell HM, Chesnut DB (1958) Theory of Isotropic Hyperfine Interactions in PiElectron Radicals. J Chem Phys 28:107-117.

76. Heller C, McConnell HM (1960) Radiation Damage in Organic Crystals. 2. Electron Spin Resonance of $\left(\mathrm{CO}_{2} \mathrm{H}\right) \mathrm{CH}_{2} \mathrm{CH}\left(\mathrm{CO}_{2} \mathrm{H}\right)$ in Beta-Succinic Acid. J Chem Phys 32:1535-1539.

77. Morton JR (1964) Electron Spin Resonance of Oriented Radicals. Chem Rev 64:453-471.

78. Bernhard WA, Close DM, Mercer KR, Corelli JC (1976) ESR of X-Irradiated SingleCrystals of 3'-Cytidylic Acid - Hydrogen Abstraction from C5' of Sugar Moiety. Radiat Res 66:19-32.

79. Vestad TA, Gustafsson H, Lund A, Hole EO, Sagstuen E (2004) Radiation-Induced Radicals in Lithium Formate Monohydrate $\left(\mathrm{LiHCO}_{2} \cdot \mathrm{H}_{2} \mathrm{O}\right)$. EPR and ENDOR Studies of XIrradiated Crystal and Polycrystalline Samples. Phys Chem Chem Phys 6:3017-3022.

80. Ko CL, Box HC (1978) Exchangeable Proton Couplings in Free-Radicals - Radiation Products of Hydroxyproline HCl. J Chem Phys 68:5357-5362. 
81. Tarpan MA (2011) Electron Magnetic Resonance Study of the Structure and Thermal Stability of Radiation-Induced Radicals in Fructose and Trehalose. PhD Thesis, Ghent University.

82. McConnell HM, Heller C, Cole T, Fessenden RW (1960) Radiation Damage in Organic Crystals I. CH(COOH $)_{2}$ in Malonic Acid. J Am Chem Soc 82:766-775.

83. McConnell HM, Robertson RE (1957) Spectroscopic Splitting Factors in Aromatic Radicals. J Phys Chem 61:1018.

84. Bernhard WA, Close DM, Hüttermann J, Zehner H (1977) Alkoxy Radical, $\mathrm{RCH}_{2} \mathrm{O}$, as a Free-Radical Product in X-Irradiated Single Crystals of Nucleosides and Nucleotides. J Chem Phys 67:1211-1219.

85. Samskog PO, Lund A (1980) The Alkoxy Radical RCHO Formed in Irradiated SingleCrystals of Rhamnose. Chem Phys Lett 75:525-527.

86. Lee JY, Box HC (1973) ESR and ENDOR Studies of DL-Serine Irradiated at 4.2 K. J Chem Phys 59:2509-2512.

87. Box HC, Budzinski EE (1975) Primary Radiation Damage in Thymidine. J Chem Phys 62:197-199.

88. Locher SE, Box HC (1980) ESR-ENDOR Studies of X-Irradiated Glucose-1-Phosphate Dipotassium Salt. J Chem Phys 72:828-832.

89. Pauwels E, Lahorte P, Vanhaelewyn G, Callens F, De Proft F, Geerlings P, Waroquier M (2002) Tentative Structures for the Radiation-Induced Radicals in Crystalline Beta-DFructose Using Density Functional Theory. J Phys Chem A 105:12340-12348.

90. Pauwels E, Van Speybroeck V, Vanhaelewyn G, Callens F, Waroquier M (2004) DFTEPR Study of Radiation-Induced Radicals in Alpha-D-Glucose. Int J Quantum Chem 99:102-108.

91. Vanhaelewyn GCAM, Pauwels E, Callens FJ, Waroquier M, Sagstuen E, Matthys PFAE (2006) Q-band EPR and ENDOR of Low Temperature X-Irradiated Beta-D-Fructose Single crystals. J Phys Chem A 110:2147-2156.

92. Pauwels E, Van Speybroeck V, Waroquier M (2006) Study of Rhamnose Radicals in the Solid State Adopting a Density Functional Theory Cluster Approach. J Phys Chem A 110:6504-6513.

93. Car R, Parrinello M (1985) Unified Approach for Molecular-Dynamics and DensityFunctional Theory. Phys Rev Lett 55:2472-2474.

94. CPMD V3.11, Copyright IBM Corp., 1990-2006, Copyright MPI für Festkörperforschung, Stuttgart, 1997-2001.

95. Lippert G, Hutter J, Parrinello M (1997) A Hybrid Gaussian and Plane Wave Density Functional Scheme. Mol Phys 92:477-487.

96. Lippert G, Hutter J, Parrinello M (1999) The Gaussian and Augmented-Plane-Wave Density Functional Method for Ab Initio Molecular Dynamics Simulations. Theo Chem Acc 103:124-140

97. http://www.cp2k.org/

98. Declerck R, Pauwels E, Van Speybroeck V, Waroquier M (2006) First-Principles Calculations of Hyperfine Parameters with the Gaussian and Augmented-Plane-Wave Method: Application to Radicals Embedded in a Crystalline Environment. Phys Rev B 74:art. no. 245103.

99. Declerck R, Van Speybroeck V, Waroquier M (2006) First-Principles Calculation of the EPR $g$ Tensor in Extended Periodic Systems. Phys Rev B 73:art. no. 115113

100. Weber V, Iannuzzi M, Giani S, Hutter J, Declerck R, Waroquier M (2009) Magnetic Linear Response Properties Calculations with the Gaussian and Augmented-Plane-Wave Method. J Chem Phys 131:art. no. 014106.

101. Tarpan MA, De Cooman H, Hole EO, Waroquier M, Callens F (2012) Radiation Products at $77 \mathrm{~K}$ in Trehalose Single Crystals: EMR and DFT Analysis. J Phys Chem A 116:33773387. 
102. Kevorkyants R, Wang X, Close DM, Pavanello M (2013) Calculating Hyperfine Couplings in Large Ionic Crystals Containing Hundreds of QM Atoms: Subsystem DFT Is the Key. J Phys Chem B 117:13967-13974.

103. De Cooman H (2009) A Combined EMR and DFT Study of Radiation-Induced Defects in Sucrose and Glucose 1-Phosphate. PhD Thesis, Ghent University.

104. De Cooman $\mathrm{H}$ et al. Unpublished Results (K-band EMR Study of $6 \mathrm{~K}$ Irradiated Sucrose).

105. De Cooman $\mathrm{H}$ et al. Unpublished Results (X-band EMR Study of 80K Irradiated Sucrose).

106. Kusakovskij J (2013). Exploring the Possibilities of EPR Methods for Identifying Radiation Damage to Solids: Application to Sucrose and Silicon. Master Thesis, Vilnius University [in Lithuanian].

107. Box HC, Budzinski EE (1983) A Variation of the Alkoxy Radical. J Chem Phys 79:4142_ 4145.

108. Budzinski EE, Potter WR, Potienko G, Box HC (1979) Characteristics of Trapped Electrons and Electron Traps in Single Crystals. J Chem Phys 70:5040-5044.

109. Box HC, Budzinski EE, Freund HG (1990) Studies of Electrons Trapped in X-Irradiated Rhamnose Crystals. J Chem Phys 93:262-266.

110. Kevan L, Schlick S, Narayana PA, Feng DF (1981) Application of the Semicontinuum Potential Model to Deduce Localized Electron Trapping Sites in Single Crystals of DSorbitol. J Chem Phys 75:1980-1983.

111. Samskog PO, Kispert LD, Lund A (1983) Geometric Model of Trapped Electrons in Trehalose Single Crystals X Ray Irradiated at 3 K. An EPR Study. J Chem Phys 78:57905794.

112. Samskog PO, Kispert LD, Lund A (1983) Geometric Model of Trapped Electrons in XRay-Irradiated Single-Crystals of Rhamnose. J Chem Phys 79:635-638.

113. Ueda H (1963) Electron Spin Resonance Studies of Irradiated Single Crystals of Sugars. J Phys Chem 67:2185-2190.

114. Moens P, Devolder P, Hoogewijs R, Callens F, Verbeeck R (1993) Maximum-Likelihood Common-Factor Analysis as a Powerful Tool in Decomposing Multicomponent EPR Powder Spectra. J Magn Reson Ser A 101:1-15.

115. Bungum B (1992) Radiation-Induced Radicals in Glucose-1-Phosphate Studied with ESR and ENDOR Techniques. Master Thesis, University of Oslo [in Norwegian].

116. De Cooman H, Vanhaelewyn G, Pauwels E, Sagstuen E, Waroquier M, Callens F (2008) Radiation-Induced Radicals in Glucose-1-phosphate. I. Electron Paramagnetic Resonance and Electron Nuclear Double Resonance Analysis of in situ X-Irradiated Single Crystals at 77 K. J Phys Chem B 112:15045-15053.

117. Narendra N, Visvamitra MA (1984) Structure of the Dipotassium Glucose 1-Phosphate Dihydrate $\mathrm{C}_{6} \mathrm{H}_{11} \mathrm{O}_{9} \mathrm{P}^{2-} .2 \mathrm{~K}^{+} .2 \mathrm{H}_{2} \mathrm{O}$. Curr Sci 53:1018-1020.

118. Bungum B, Hole EO, Sagstuen E, Lindgren M (1994) Electron-Paramagnetic-Resonance of X-Irradiated Sodium and Potassium-Salts of Glucose-1-Phosphate - Identification of $\mathrm{PO}_{3}{ }^{2-}$ Radicals at Room-Temperature. Radiat Res 139:194-202.

119. Sanderud A, Sagstuen E (1996) EPR Study of X-Irradiated Hydroxyalkyl Phosphate Esters - Phosphate Radical Formation in Polycrystalline Glucose Phosphate, Ribose Phosphate and Glycerol Phosphate Salts at 77 and 295 K. J Chem Soc Faraday Trans 92:995-999.

120. Samskog PO, Kispert LD, Lund A (1982) An Electron-Spin-Resonance Study of $77 \mathrm{~K}$ Alkoxy and Hydroxyalkyl Radicals in X-Ray-Irradiated Trehalose Single-Crystals. J Chem Phys 77:2330-2335.

121. Ueda H (1963) Electron Spin Resonance studies of irradiated single crystals of D-fructose and L-sorbose. J Phys Chem 67:966-968.

122. Vanhaelewyn G, Lahorte P, De Proft F, Mondelaers W, Geerlings P, Callens F (2001) Electron magnetic resonance study of stable radicals in irradiated D-fructose single crystals. Phys Chem Chem Phys 3:1729-1735. 
123. Vanhaelewyn GCAM, Jansen B, Callens FJ, Sagstuen E (2004) ENDOR-Assisted Study of the Stable EPR Spectrum of X-Irradiated $\alpha$-L-Sorbose Single Crystals: MLCFA and Simulation Decomposition Analyses. Radiat Res 162:96-104.

124. Jansen B (1992) Radiation Induced Radicals in Single Crystals of $\alpha$-L-Sorbose. ESR-, ENDOR, and Pulse Radiolysis Studies at Room Temperature. Master Thesis, University of Oslo [in Norwegian].

125. von Sonntag C (1987) The Chemical Basis of Radiation Biology. Taylor\&Francis, London.

126. Chatgilialogly C (2009) Reactivity of Nucleic Acid Sugar Radicals. In: Radical and Radical Ion Reactivity in Nucleic Acid Chemistry (Greenberg MM, Ed.). Ch. 4. Wiley, New York.

127. Pauwels E, De Cooman H, Waroquier M, Hole EO, Sagstuen E (2014) Solved? The Reductive Radiation Chemistry of Alanine. Phys Chem Chem Phys 16:2475-2481.

128. Sagstuen E, Hole EO, Sanderud A (2004) The Solid State Radiation Chemistry of Simple Amino Acids, Revisited. Radiat Res 162:112-119.

129. Bernhard WA (2009) Radical Reaction Pathways Initiated by Direct Energy Deposition in DNA by Ionizing Radiation. In: Radical and Radical Ion Reactivity in Nucleic Acid Chemistry (Greenberg MM, Ed.). Ch. 2. Wiley, New York.

130. Bernhard WA and Close DM (2004) DNA Damage Dictates the Biological Consequences of Ionizing Radiation: The Chemical Pathways. In: Charged Particle and Photon Interactions with Matter (Mozumder A, Hatano Y Eds.). Ch. 15. Marcel Dekker, New York.

131. Attix FH (1986) Introduction to Radiological Physics and Radiation Dosimetry. Wiley, New York.

132. Sanche L (2009) Low-Energy Electron Interaction with DNA: Bond Dissociations and Formation of Transient Anion, Radicals and Radical ions. In: Radical and Radical Ion Reactivity in Nucleic Acid Chemistry (Greenberg MM Ed.). Ch. 8. Wiley, New York.

133. Bass AD, Sanche L (2004) Interactions of Low Energy Electrons with Atomic and Molecular Solids. In: Charged Particle and Photon Interactions with Matter (Mozumder A, Hatano Y Eds.). Ch. 9. Marcel Dekker, New York.

134. Lund A, Schlick S (1989) Trapped Electrons in Crystalline Media. Res Chem Intermed 11:37-66.

135. Debije MG, Milano MT and Bernhard WA (1999) DNA Responds to Ionizing Radiation as an Insulator, not as a 'Molecular Wire'. Angew Chem Int Ed 38:2752-2756.

136. Krivokapic A, Herak JN, Sagstuen E (2008) Proton-Coupled Hole Transfer in X-Irradiated Doped Crystalline Cytosine. $\mathrm{H}_{2} \mathrm{O}$. J Phys Chem A 112:3597-3606.

137. Löfroth G, Gejvall T (1974) Radiation Induced Effects on Exchangeable Hydrogens in Crystalline Solids. Acta Chem Scand B 28:777-780.

138. Hart EJ, Anbar M (1970) The Hydrated Electron. Wiley, New York.

139. Kuper GC, Whitfield GD (1963) Polarons and Excitons. Oliver and Boyd, Edinburgh.

140. Sagstuen E, Lindgren M, Lund A (1991) Electron Trapping and Reactions in Rhamnose by ESR and ENDOR. Radiat Res 128:235-242.

141. Steenken S (1989) Purine Bases, Nucleosides, and Nucleotides: Aqueous Solution Redox Chemistry and Transformation Reactions of Their Radical Cations and $\mathrm{e}^{-}$and $\mathrm{OH}$ Adducts. Chem Rev 89:503-520.

142. Grigoriev EI, Trakhtenberg LI (1996) Radiation Chemical Processes in Solid Phase - Theory and Applications. CRC Press, Boca Raton.

143. Madden KP, Bernhard WA (1980) A 1,2 Hydrogen Shift and Other Thermally Induced Free Radical Reactions in X-Irradiated Methyl-Alpha-D-Glucopyranoside Single Crystals. An ESR-ENDOR Study. J Phys Chem 84:1712-1717.

144. Box HC, Budzinski EE and Freund HG (1984) The gamut of alkoxy radicals. J Chem Phys $81: 4898-4900$

145. Aalbergsjø S, Pauwels E, Hole EO, Sagstuen E, work in progress. 